Hole burning imaging studies of cancerous and analogous normal ovarian tissues utilizing organelle specific dyes

by

\title{
Satoshi Matsuzaki
}

A dissertation submitted to the graduate faculty .

in partial fulfillment of the requirements for the degree of

DOCTOR OF PHILOSOPHY

\author{
Major: Physical Chemistry \\ Program of Study Committee: \\ Ryszard Jankowiak, Co-major Professor \\ Xueyu Song, Co-major Professor \\ Mei Hong \\ James W. Evans \\ Mark S. Hargrove
}

Iowa State University

Ames, lowa

2004

Copyright $(\mathcal{O}$ Satoshi Matsuzaki, 2004. All rights reserved. 


\section{Graduate College \\ Iowa State University}

This is to certify that the doctoral dissertation of

Satoshi Matsuzaki

has met the dissertation requirements of Iowa State University

\begin{tabular}{c}
\hline Co-major Professor \\
\hline Co-major Professor \\
\hline
\end{tabular}

For the Major Program 


\section{TABLE OF CONTENTS}

CHAPTER 1. GENERAL INTRODUCTION I

CHAPTER 2. BIOLOGICAL ASPECTS $\quad 8$

2.1 Mitochondria 9

2.2 Membranes of Cells 15

2.2.1 Structure and Function of Cell Membranes 15

2.2.2 Electrical Properties of Membranes 20

2.3 Mitochondrial Membrane Potential 23

2.3.1 Monitoring in situ Mitochondrial Membrane Potential 25

2.3.2 $\Delta \Psi_{\text {in }}$ Monitoring in Non-quench Mode 26

2.3.3 $\Delta \Psi_{\text {In }}$ Monitoring in Quench Mode 28

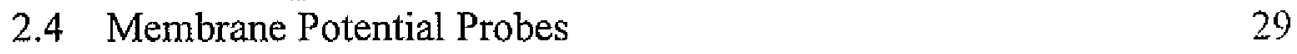

2.4.1 Autofluorescense $\quad 30$

2.4.2 Characterization of MF680 32

CHAPTER 3. INTRODUCTION TO HOLE BURNING IMAGING 41

3.1 Introduction to Nonphotochemical Hole Burning 4I

3.2 Hole Burning Mechanisms 49

3.2.1 Dispersive Hole Growth Kinetics $\quad 52$

3.2.2 Temperature Dependence of Electronic Dephasing 56

3.3 Effects of External Electric (Stark) Fields 58

3.4 Application of NPHB to Biological Anomaly Detection 62

CHAPTER 4. SINGLE-CELL NONPHOTOCHEMICAL HOLE BURNING OF OVARIAN SURFACE EPITHELIAL CARCINOMA AND NORMAL CELLS

Abstract

Introduction 67

$\begin{array}{ll}\text { Materials and Methods } & 71\end{array}$

$\begin{array}{ll}\text { Results and Discussion } & 73\end{array}$

Acknowledgments $\quad 84$

References $\quad 84$

CHAPTER 5. NONPHOTOCHEMICAL HOLE BURNING STUDY OF A MITOCHONDRIAL SELECTIVE RHODAMINE DYE IN CANCEROUS AND NORMAL HUMAN OVARIAN TISSUES 86 $\begin{array}{ll}\text { Abstract } & 86\end{array}$ $\begin{array}{ll}\text { Introduction } & 87\end{array}$

Experimental 91

Results and Discussion $\quad 94$

$\begin{array}{ll}\text { Acknowledgments } & 109\end{array}$ 
CHAPTER 6. GENERAL CONCLUSIONS

APPENDIX B. FLOW CYTOMETRIC ESTIMATION OF MITOCHONDRIAL MEMBRANE POTENTIAL 


\section{CHAPTER 1. GENERAL INTRODUCTION}

Cancer is a group of diseases characterized by the uncontrolled growth and spread of abnormal cells. If the spread is not controlled, it can result in death. Cancer is caused by both external factors (tobacco, chemical, radiation, and infectious organisms) and internal factors (inherited mutations, hormones, immune conditions and mutations that occur from the metabolic process). These causal factors may act together or in sequence to initiate or promote carcinogenesis. When the DNA of a cell undergoes several mutations whereby a dividing cell sustains a mutation and, subsequently passes this mutation onto its daughter cells, a malignant tumor is formed. These cells then sustain further mutations that are passed onto the next generation of cells, and so on, until this system develops an inclination to carry on uncontrolled proliferation. At a certain state, when the cells begin their excessive proliferation, they may still appear normal. Though this state persists only until another mutation causes a group of cells to assume irregular shapes and orientations which will eventually develop into an in situ cancer. Once the tumor cells become invasive, they can eventually leave the primary site and metastasize by entering the blood stream or the lymphatic system and, thus initiate the growth of other tumors at secondary sites. It is at this point that the tumor is said to be malignant and incurable $[1,2]$.

The American Cancer Society (ACS) projects that $1,368,030$ new cases of cancer will be diagnosed in the United States in 2004, with 25,580 new cases of ovarian cancer [3]. These estimates do not include basal and squamous cell skin cancers. All cancer caused by cigarette smoking and heavy use of alcohol could be prevented completely. The ACS estimates that in 2004 more than 180,000 cancer deaths are expected to be caused by tobacco use. Evidence suggests that about one-third of the 563,700 cancer deaths expected to occur 
in 2004 will be related to nutrition, physical inactivity, excessive weight or obesity, and other lifestyle factors, and thus could also be prevented [3]. In addition, many of the more than 1 million skin cancers that are expected to be diagnosed in 2004 could have been prevented by adequate protection from the sun's rays. Regular screening by a qualified health care professional can result in the detection of cancers (of the breast, colon, rectum, cervix, prostate, oral cavity, and skin) at earlier stages, when treatment is more likely to be successful. Cancers that can be detected earlier by screening account for about half of all new cancer cases. The 5-year relative survival rate for these cancers is about $84 \%$. If all of these cancers were diagnosed at a localized stage through regular cancer screening, 5-year survival would increase to about $95 \%[3,4]$. In this context, prevention includes the aforementioned guidelines determined by the ACS as well as early detection of cancers when it is at a curable stage.

The potential problem with screening techniques is the possibility of obtaining galesnegative or false-positive results, which incorrectly indicate the absence or presence of cancer, respectively. The utility of a screening technique is determined by its level of accuracy. The degree of accuracy is, in turn, characterized by the sensitivity and specificity of a particular methodology. Sensitivity describes the ability of a screening test to identify a disease whereby an increase in sensitivity decreases the chance of false-negative results. For instance, the accuracy of a pap smear test, which is a commonly used test for cervical cancer, can vary from $60-99 \%$ and becomes less reliable in older women, especially those who are post-menopausal [5]. In addition, specificity also refers to a test's ability to exclude detection of a disease that is not present thereby decreasing the possibility of a false-positive outcome. Prostate cancer, for instance, is curable when detected at an early stage, but 
because it might not produce symptoms, $25 \%$ of diagnoses occur at late stages. Screening for elevated levels of PSA have been suggested for early detection of prostate cancer, though this test is prone to giving false-positive results because $40 \%$ of men with elevated PSA levels do not have prostate cancer [4]. In general, many methods for screening cancer are available, each having its own degree of accuracy.

The presence of various symptoms, such as rapid weight loss, fever, and fatigue [6], can implicate many possible afflictions, including cancer. The diagnostic test used to detect cancer will depend on the symptoms, possible location and type of cancer, patient and physician preferences, and the willingness of a person's health care provider to pay for conducting a particular test [7]. Moreover, since no single methodology can accurately diagnose, grade, and stage a tumor, techniques are often used in combination with each other.

Conventional and digital radiography are inexpensive and easy imaging methodologies used for the initial evaluation of patients. Both methods are based on differences in density between normal and cancerous tissues and are used to image deep tumors. Conventional radiography involves capturing images on $\mathrm{x}$-ray film while images obtained by digital radiography are captured and stored on computer. Furthermore, conventional $\mathrm{x}$-rays usually require a contrast medium such as barium to be administered to the patient in order to enhance the contrast between normal and tumor tissues, while the contrast medium is often times unnecessary for digital radiography. Unlike the radiographic techniques, computerized tomography (CT) uses x-rays to provide three-dimensional images of a tumor, instead of sliced planar images. These three-dimensional images furnish information about tumor size, shape, volume, and location [8]. However, CT scanning is 
much more costly than the aforementioned radiographic techniques and is unable to detect small tumors where no mass has developed.

Magnetic resonance imaging (MRI) uses radiowaves and magnetic fields to produce two-dimensional images. The biggest advantage of MRI over x-rays is that it can be used to image regions of the body that $\mathrm{x}$-rays cannot, such as the brain. Furthermore, MRI can detect smaller tumors than $\mathrm{CT}$ and is also able to distinguish between tumors and cysts [8]. On the other hand, the disadvantage of MRI is that it is unable to detect calcification, which is a potential sign of breast cancer. The high cost of MRI, which is even more expensive than $\mathrm{CT}$, also prohibits it from being widely available. Fortunately, an alternative technique known as ultrasonagraphy (or ultrasound) is readily available and inexpensive. Like MRI, ultrasound can distinguish between solid tumor masses and cysts, which distinguishes it as a good technique, for instance, for visualizing solid tumors in soft tissues. However, ultrasound is unable to penetrate bone and gas-filled cavities and is, thus ineffective in imaging cancer of the brain, lungs, and the gastrointestinal tract [9]. More recently, other advanced imaging techniques such as positron-emission tomography (PET) and singlephoton-emission computed tomography (SPECT) started to get radiologists' attention. Although these methods can produce 3-D images of physiological functions such as blood flow, oxygen consumption or glucose metabolism, they do not show either bones or organs directly [8]. It should be noted that PET and SPECT also have limited resolution so that the increase in functional information about blood flow and cell metabolism is counterbalanced by the loss of precise locational information.

The diagnostic imaging techniques mentioned so far are all noninvasive. In contrast, biopsies, dubbed the "end-all" diagnostic technique, are invasive, involving the removal of 
suspect tissue samples. Methods for obtaining these samples include fine needle biopsies and surgical biopsies whereby excised tissue is prepared for examination by a pathologist. In fine needle biopsies, a needle is used to remove easily accessible tumors and in some cases, deep tumors. Unfortunately, fine needle biopsies often yield false-negative results in the case of the needle missing the tumor [5]. This is especially problematic for deep tumors and, as a result, other imaging techniques such as ultrasound are used in conjunction with needle biopsies to aid in directing the needle to the tumor mass. Surgical biopsies are considered more complicated procedures and are the most invasive, often requiring a patient to undergo two separate surgical procedures. Though, the advantage of surgical biopsies over needle biopsies is that more tissue can be removed for examination, thus reducing the risk of an incorrect diagnosis [7]. Some surgical biopsies involve the preparation of frozen slices of tissue that can be immediately examined. In this way, a patient undergoes surgery only once since a prompt examination of the excised tissue allows for immediate excision of the tumor. The problems arising from frozen tissue sections comes as a result of the distortion of the tissue due to freezing, thereby making an analysis of these sections difficult. Note that endoscopic methods are used for obtaining tissue samples for biopsy from otherwise inaccessible internal organs. In all cases, the accuracy of biopsies is subject to the expertise of the pathologist examining the tissue samples.

The basic premise of the work presented herein is centered upon the investigation of better cancer detection techniques which will lead to improved detection rates, with earlier diagnosis being the ultimate objective. The research presented in this dissertation seeks to improve and add to cancer detection methods currently in use, and the means by which to accomplish this is the application of nonphotochemical hole burning (NPHB) imaging. 
NPHB is the optical analog of MRI, whereby pure optical dephasing times $\left(T_{2}^{*}\right)$ are measured instead of proton relaxation times $\left(T_{1}\right)$, with the common relation existing between the two in total dephasing time $\left(T_{2}\right)$. Note that recently Furusawa and coworkers [10], by applying photon echo spectroscopy to normal and cancerous human kidney tissues stained with rhodamine $\mathrm{X}$ isothiocyanate, were able to distinguish between the two tissue types based on $T_{2}$ dephasing times for the tissues. NPHB is inherently sensitive to the nanoenvironment in which a fluorophore resides. In practice, easily measured parameters obtained from the rate of hole growth, i.e., hole growth kinetics (HGK), can be compared to distinguish the environments surrounding the fluorophores. In addition to HGK, application of an external electric (Stark) field to holes can also be used to differentiate matrix induced dipole moment changes. The objective of the work presented here will contain the following: to find and target cellular structural / bioenergetics differences between normal and cancerous cells/tissues, and to present the ability of NPHB imaging for resolving and quantifying those differences.

The organization of this dissertation will be as follows: Chapter two discusses pertinent biological aspects concerned with the research presented in chapter four, including discussions for the structure and the bioenergetics of the mitochondrial membrane. Chapter three gives an overview of the aspects of hole burning spectroscopy for its application in the study of cellular systems. Chapter four is a previously published paper which presents the single-cell hole burning results of ovarian surface epithelial carcinoma and normal cells stained with the mitochondrion-specific rhodamine dye MF680. Chapter five is a manuscript submitted for publication pertaining to the results obtained from the application of hole burning imaging to human ovarian tissues. In this manuscript, cancerous tissue taken from 
the ovarian surface and analogous normal peritoneal tissue were stained with MF680 for hole-burning imaging in order to elucidate the differences in in vitro tissue samples. Chapter six concludes the work presented and discusses the possible future experimentation. Appendix A presents another mitochondrion-specific probe candidate for use in future hole burning imaging studies of the mitochondrial membrane potential. Appendix B introduces a semi-quantitative mitochondrial membrane potential determination technique via the flow cytometry. Lastly, Appendix $\mathrm{C}$ discusses procedures and the validity of cryopreservation for cell/tissue samples.

\section{References:}

1. Weinberg, R. Sci. Am. 1996, 275, 62 .

2. Weinberg, R. One Renegade Cell: How Cancer Begins, 1998, Basic Books, New York.

3. American Cancer Society, Cancer Facts and Figures, 2004.

4. American Cancer Society, Cancer Prevention and Early Detection Facts and Figures, 2004.

5. Richards-Kortum, R., Sevick-Muraca, E. Annu. Rev. Phys. Chem., 1996, 47, 555.

6. Eyre, H. J., Lange, D. P., Morris, L. B. Informed Decisions: The Complete Book of Cancer Diagnosis, Treatment, and Recovery ( $2^{\text {nd }}$ edn.), 2002, American Cancer Society.

7. Sidransky, D. Sci. Am. 1996, 275, 104.

8. Giger, M. L., Pelizzari, C. A. Sci. Am. 1996, 275, 110.

9. Udupa, J. K., Herman, G. T. 3D Imaging in Medicine, 1991, CRC Press.

1.0. Furusawa, A., Suga, T., Uchikawa, K. J. Opt. Soc. Am. B. 1994, 11, 1456. 


\section{CHAPTER 2. BIOLOGICAL ASPECTS}

Mitochondria, the site of cellular oxidative phosphorylation, supply ATP to the eukaryotic cells for their constant energy demand during functions such as biosynthesis, muscular contraction, ion and solute pumping. The mitochondrial respiratory chain gives rise to an electrochemical proton gradient across the inner mitochondrial membrane, which can be broken down into its transmembrane electrical potential $\left(\Delta \Psi_{m}\right)$ component and its $\mathrm{pH}$ gradient component. The energy stored in this electrochemical gradient is the driving force for the proton flux through the $\mathrm{F}_{\mathrm{O}} \mathrm{F}_{\mathrm{F}} \mathrm{ATPase}$ of the inner mitochondrial membrane as well as adenosine triphosphate (ATP) synthesis [1] (see Section 2.1 for further discussion). As a consequence $\Delta \Psi_{m}$ and the ATP demand are closely linked.

Measurement of $\Delta \Psi_{\mathrm{m}}$ in intact living cells has been made possible by several techniques using lipophilic cationic dyes that accumulate electrophoretically in the strongly negatively charged matrix of mitochondria [2]. This redistribution of dye was shown to be primarily governed by the Nernst equation [3]. Semi-quantitative $\Delta \Psi_{m}$ measurements of ovarian surface epithelial cells have been previously performed by Professor Small's group $[4,5]$ using this approach in conjunction with the hole burning imaging technique. This study revealed that the difference in the permanent dipole moment changes of a Nernstian dye bound to the mitochondrial inner-membrane of two cell lines corresponds to the difference in $\Delta \Psi_{m}$ of the cell lines as measured in another manner. This raised the question of whether $\Delta \Psi_{m}{ }^{\prime} s$ can be detected for in vitro tissue samples, whereby through conventional methods it is impossible to monitor $\Delta \Psi_{\mathrm{m}}$ quantitatively, to elucidate the differences between cancerous and normal tissues. 
The work presented in this thesis could not have been made possible except for the extraordinarily unique cellular organelle present in nearly every type of cell in the human body: mitochondria. As such, a large portion of this chapter will be devoted to the history of research on this structure and the bioenergetics of the mitochondrial membrane. The remainder of the chapter will be devoted to the discussion of membrane potential probes.

\subsection{Mitochondria}

A review by Cowdry in 1918, quoted by Lehninger [6], contains more than a dozen terms referring to structures we now identify as mitochondria: blepharoblasts, chondriomites, chondriosomes, fuchsinophilic granules, Korner, Fadenkorper, mitogel, parabasal bodies, plastochondria, plastosomes, vermicules, sarcosomes, bioblasts, and so on. Beginning around 1850, microscopist used the words chondros (Greek), grain (English), and Korn (German) in their descriptions of the morphology of distinct cell structures. Improvements in staining yielded more accurate morphological descriptions, and in some tissues the "grains" were seen as "threads" (Faden in German, mitos in Greek), hence Fadenkorper or mitochondria (noted by Benda [7] in 1898). Although it is common knowledge today, it is quite interesting to note that in as early as 1890 Altman, who referred to these granules as "bioplasts" in a book on Elementarorganismen, proposed that these granules were actually autonomous, elemental living units, forming bacterialike colonies in the cytoplasm of the host cell. In 1912 and 1913, Kingsbury and Warburg independently found that these granular, insoluble subcellular structures were in fact associated with respiration, which challenged the previously held theory of their role in genetics. Though it was not for another 
thirty to forty years of intense and painstaking biochemical analyses before the mitochondria were finally classified as the "powerhouse of the cell."

The structure of the mitochondria was elucidated for the first time with the advent of the transmission electron microscope. A schematic representation of the mitochondrial structure is given in Figure 2.1.1. The potential complexity of this organelle became apparent from the earliest electron microscopic observations, which revealed the existence of an inner and an outer membrane [8,9], with the inner membrane often highly folded. Convolutions of the inner membrane into the center of the mitochondrial body give rise to the most prominent feature of mitochondria, the cristae (termed by Palade [10-12]). The morphology of the cristae serves to increase the surface area of the inner membrane where the proteins responsible for phosphorylation reside. (The theoretical gain in surface area can be roughly estimated, assuming a surface area of $166.6 \mu \mathrm{m}^{2}$ for a lipid bilayer membrane $0.006 \mu \mathrm{m}$ thick and a minimum surface area of $4.83 \mu \mathrm{m}^{2}$ which would cover a sphere of 1 $\mu \mathrm{m}^{3}$ volume with a radius $\mathrm{r}=0.62 \mu \mathrm{m}$, to be $\sim 35$ fold increase.) Estimates of the inner membrane surface area to unit volume ratio for rat liver mitochondria are $\sim 16.5$ and $\sim 61$ for rat heart mitochondria [13]. It then follows that the number of cristae is generally higher in tissues which experience high energy demands, i.e. muscle and neurons [14].

It is important to mention that by enacting a proton gradient between the mitochondial matrix and the intermitochondrial membrane, mitochondria generate a membrane potential. This is important for two reasons: (a) the membrane potential has become the focus of numerous investigations in that the magnitude of the potential has been found to differ in mitochondria derived from cancerous tissue as opposed to normal tissue $[15,16]$ and (b) the lipid-rich, anionic environment presents a favorable site for lipophilic, 


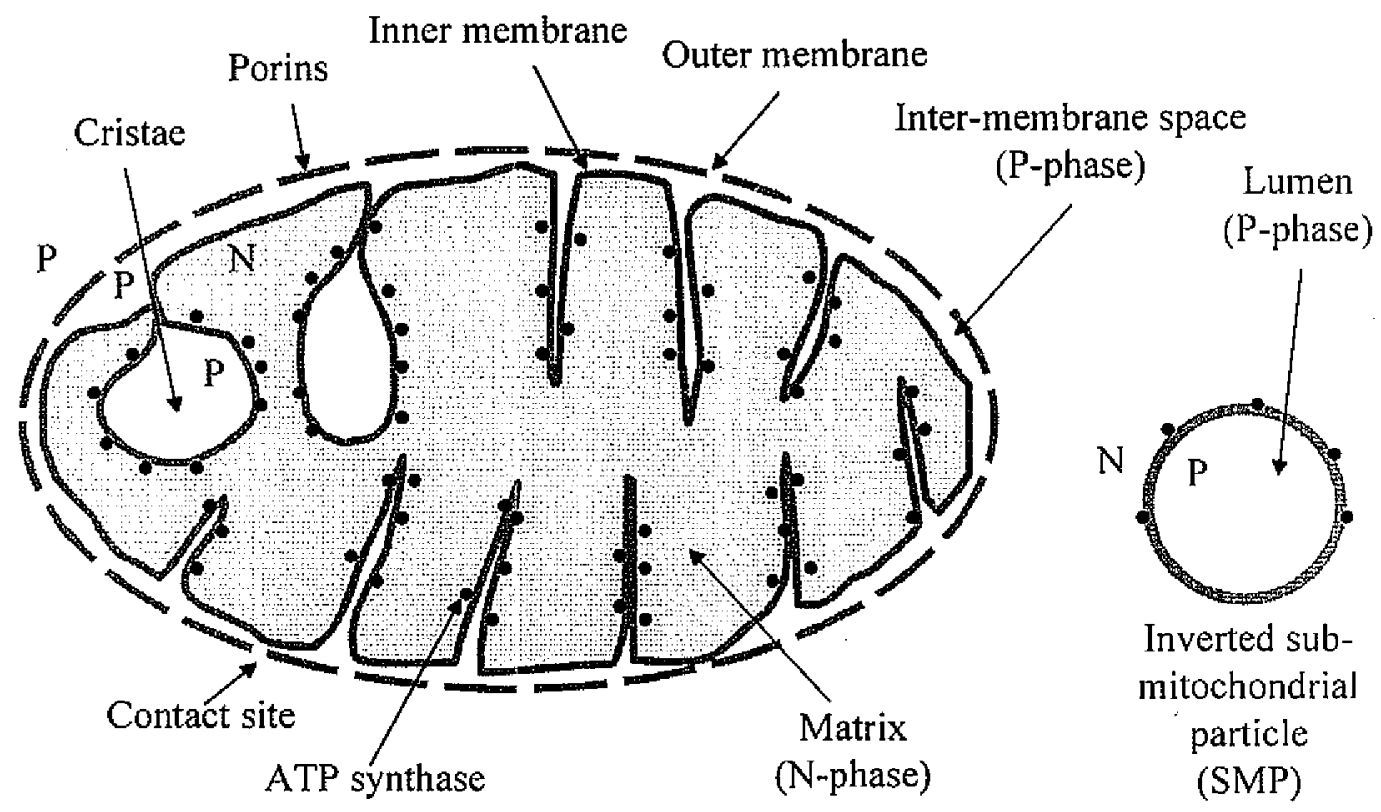

Figure 2.1.1 The schematic representation of a typical mitochondrion and submitochondrial particles. Note $\mathrm{P}$ and $\mathrm{N}$ refer to the positive and negative compartments. The shape of the cristae is highly variable and the communication between cristae and inter-membrane space may be restricted. 
carbocationic fluorescent dyes to locate [17-21]. The latter has sparked interest in such dyes for use as chemotherapeutic and photodynamic therapeutic agents.

The generation of the membrane potential is inherently related to the main function of mitochondria: that of oxidative phosphorylation, or the creation of ATP from adenosine diphosphate (ADP). The process by which this occurs inspired one of the most controversial and interesting periods in mitochondrial research history. In 1961, Paul Mitchell initially proposed the chemiosmotic theory, which attempted to explain biological energy transformation $[22,23]$. See Figure 2.1.2 for Mitchell's model. Many theories abounded as to how this process took place, and most researchers hypothesized that intermediates were formed in a coupling mechanism that eluded discovery. Mitchell proposed that such intermediates likely did not exist, and that if the oxidoreduction in the cytochrome system is organized across a membrane in an anisotropic fashion, protons can be produced on one side of the membrane and consumed on the other [13]. Respiratory-chain reactions provide the energy for the above process, with the return of protons across the membrane coupled with ATP synthesis. The driving force of ATP synthesis was postulated on the basis of both an electrical potential $(\Delta \Psi)$ and a $\mathrm{pH}$ difference $(\Delta \mathrm{pH})$ across the coupling membrane, to collectively give the proton-motive force $(\Delta p)$ :

$$
\Delta p=\Delta \Psi-\mathrm{z} \cdot \Delta \mathrm{pH}
$$

where $\mathrm{z}$ is the diffusible ion charge, which represents $2.303 \mathrm{RT} / \mathrm{F}$. The theory was not widely accepted after its introduction, but most likely due to its controversy, chemiosmosis was highly scrutinized scientifically and eventually did become the accepted theory describing the mechanism of energy coupling. 


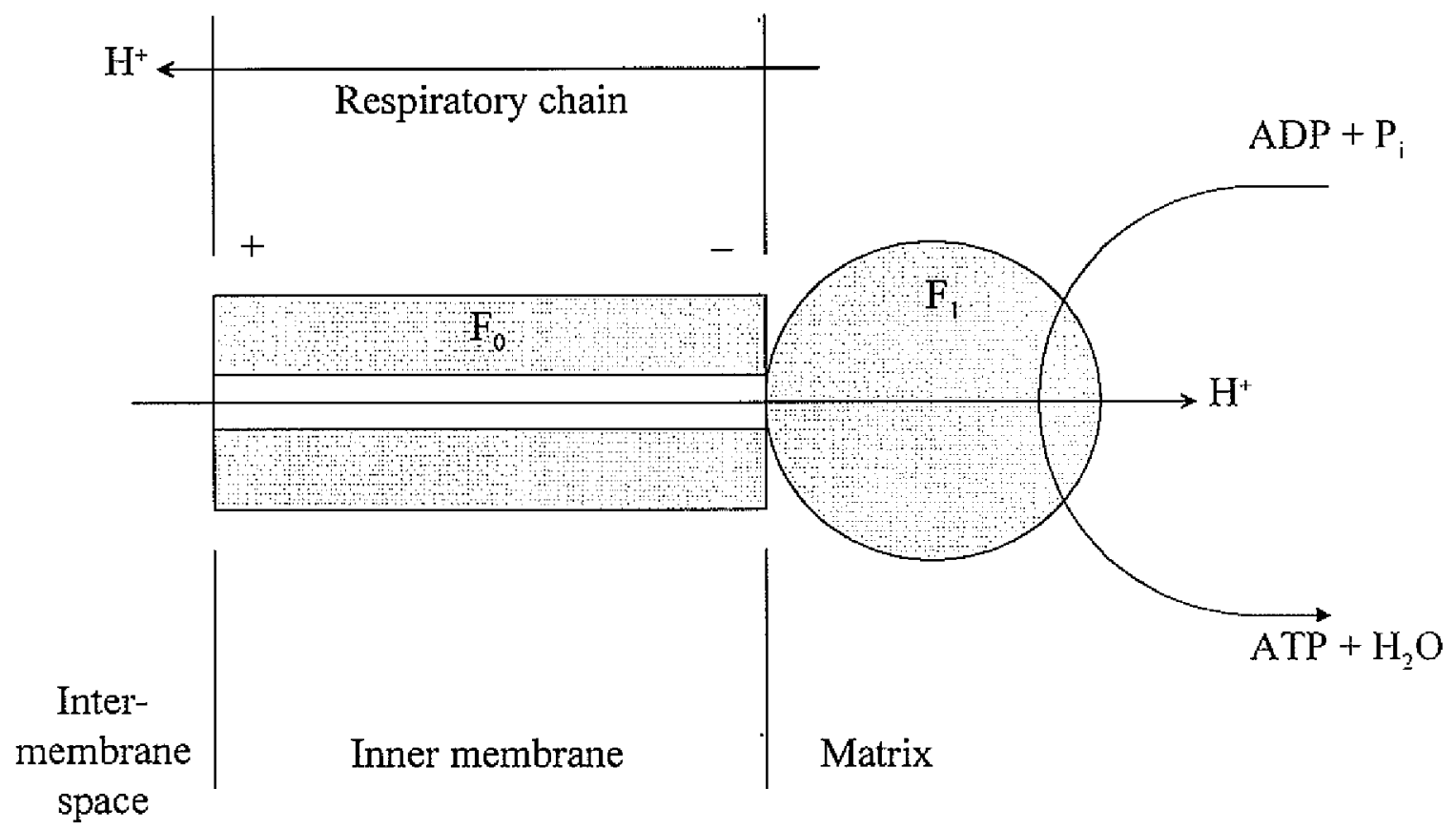

Figure 2.1.2 Mitchell's chemiosmotic theory. The respiratory chain transports protons outwards across the inner mitochondrial membrane, creating a proton gradient and membrane potential across the membrane. This electrochemical proton gradient promotes the return of protons through the ATPase $\left(\mathrm{F}_{0}\right.$ and $\mathrm{F}_{1}$ driving the synthesis of ATP by $F_{1}$ ). A similar mechanism applies to the bacterial plasma membrane. In the chloroplast thylakoid membrane, the electron transport chain drives protons inwards into the lumen so that the polarity of the system is reversed. 
It should be noted that although there may be a close resemblance between mitochondria of a given tissue, their appearance can be deviate markedly between different tissues. This raises an interesting point: mitochondria are able to change their shape, and may assume any given shape depending on the needs presented by the tissue. To be fair, however, it should be recognized that it is not fully understood whether mitochondria are in control of their shape or if forces external to the mitochondial body are what determine this. Nevertheless, despite the variability of the external shape the internal structural organization is highly consistent [24]. The structural controls and the proteins involved in oxidative phosphorylation are important to understanding the complexity of the processes which take place inside the mitochondria. Recent thorough reviews covering this are available from Yaffe [25], Wallace [26], Bereiter-Hahn [24,27], and Scheffler [14]. For our purpose, it is important to mention that alterations in mitochondria have been linked to numerous diseases, including but not limited to Parkinson's and Alzheimer's disease [28], liver disease, muscular dystrophy, cardiomyopathy, diabetes, ageing, and cancer [25,26]. In the case of cancer, it has been found that the abnormalities tend to be extraordinary and can include some or all of the following: (I) changes in size and formation of bizarre shapes, including branching, annular rings, S-shaped configurations, semicircular structures, or "giant" mitochondria; (II) changes in mitochondrial number, membrane lipid composition, and membrane potential; (III) changes in the ultra structure architecture, such as an apparent thickening of the mitochondrial cristae; (IV) alteration of the energy-linked function and the loss of electron transport components; and (V) relocation of mitochondria in the cell to abnormal positions, such as penetration of the nuclear envelope $[29,30]$. 


\subsection{Membranes of Cells}

The boundaries of cells and the organelles within are defined by their membranes. These membranes provide a critical component to cell survival by serving as a barrier to undesired substances and influences while at the same time allowing physiologically desired substances to permeate. This process facilitated by the various lipids and proteins composing the membranes allow the cell to maintain critical balances such as maintaining cellular $\mathrm{Ca}^{2+}$ homeostasis.

\subsubsection{Structure and Function of Cell Membranes}

Cell membranes are composed primarily of amphiphatic lipid molecules that have a hydrophilic head and a hydrophobic tail that, when in an aqueous solution, spontaneously form a sheet like bilayer between $\sim 50$ and $100 \AA$ thick. These molecules are arranged such that the hydrophilic heads of the lipid molecules form an interface with the water molecules of the solution while the hydrophobic tails are oriented away from the aqueous medium. The bonds between these lipid molecules are strong enough to result in membranes with low permeability for ions and most polar molecules. Because of this, proteins, e.g. ion channels, pumps and receptors, are present to regulate activity across a membrane. A general description of these membrane-associated proteins will be given in the next subsection.

Lipid molecules found in eukaryotic cells can be categorized as one of three types: phospholipids, glycolipids, and sterols. The unbranched fatty acyl chains found in phospholipids and glycolipids make up the hydrophobic tail of the lipid and are typically between 14 and 24 carbons in length. If the $\mathrm{C}-\mathrm{C}$ bonds of the fatty acyl chain are exclusively single bonds, it is referred to as a saturated fatty acid chain, whereas if one or 
more double bonds exist, it is an unsaturated fatty acid chain. Unsaturated chains adopt either a cis or trans conformation with the cis conformation being more common. The presence or absence of unsaturated fatty acyl chains within a membrane contributes to the fluidity of the membrane.

The first type of membrane lipid, known as phospholipids, is the most predominant type and can be found in all biological membranes. These molecules are derivatives of either glycerol or sphingosine serving as the lipid backbone (see Figure 2.2.1(a) and (b)). Phospholipids derived from glycerol are known as phosphoglycerides. They are formed when the two adjacent hydroxyl groups of the glycerol backbone esterify with the carboxyl ends of two fatty acid chains, and the third hydroxyl group phosphorylates, thus resulting in phosphotidate [31]. Additions of the alcohol moieties, serine, choline, ethanolamine, glycerol, and inositol to the phosphate group result in the main group of phosphoglycerides. In contrast to the many possible phospholipids derived from glycerol, sphingomyelin is the only phospholipid having a sphingosine backbone. Sphingomyelin consists of an unsaturated fatty acid chain that forms an amide bond with the sphingosine and a phosphoryl choline group esterifying the primary hydroxyl group of sphingosine (see Figure 2.2.1(b)).

Glycolipids are the second type of membrane lipid and, like sphingomyelin, are derivatives of sphingosine with an associated fatty acid chain. However, unlike sphingomyelin, the primary hydroxyl group of the sphingosine backbone contains one or more sugar groups (Figure 2.2.1(c)), which can be branched, e.g., as in gangliosides [32,33]. The third type of membrane lipid molecule is the sterol, cholesterol (Figure 2.2.1(d)). This lipid molecule is an important factor regarding the fluidity of the cell membrane. 
(a)

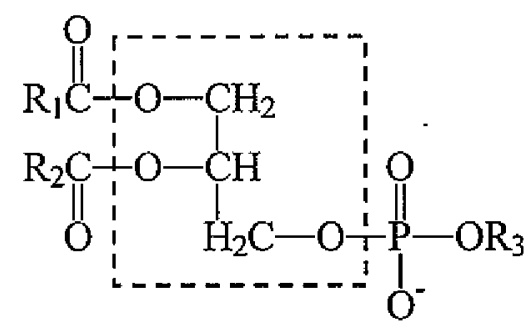

(c)<smiles>CCC(C)C=CC(O)C(CO[GaH2])NC(=O)O</smiles>

(b)

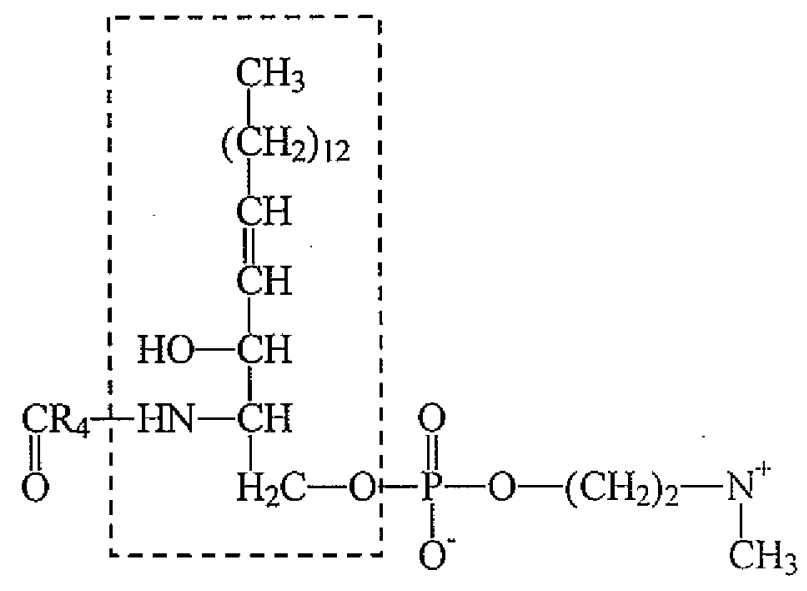

(d)<smiles>CC(C)CCC(C)C1CCC2C3CC=C4CC(O)CCC4(C)C3CCC12C</smiles>

Figure 2.2.1 The general structure of (a) phosphoglycerides, (b) sphingomyelin, (c) glycolipids and (d) cholesterol. R1 and R2 in (a) represent saturated or unsaturated fatty acyl chains, while R4 in (b) and R5 in (c) are unsaturated fatty acyl chains. R3 in (a) is an alcohol, usually serine, choline, inositol and ethanolamine [34]. The areas circled by the dashed boxes in (a) and (b) represent the lipid backbones (glycerol and sphingosine, respectively). 
In general, the lipid composition of membranes will vary with the cell type as will the distribution of lipid molecules within a particular membrane region. A heterogeneous distribution of these lipid molecules throughout the plasma membrane gives rise to physical properties of the cell membrane such as the membrane potential, which can be probed by hole burning (See Sections 3.3).

As mentioned at the beginning of this subsection, membrane-associated proteins regulate the activity that occurs across cell membranes. The specific proteins found within a cell membrane and their amounts vary with the cell type and are dependent on that particular cell's functions. Membrane-associated proteins are classified as being either integral or peripheral depending on the ability to dissociate the protein from the membrane, where integral proteins are far more difficult to dissociate than peripheral proteins. Examples of integral and peripheral proteins include ion channels and cytoskeletal proteins, respectively. See Figure 2.2.2 for the scheme. Integral proteins sometimes span the length of a membrane one or more times (proteins of this type are referred to as transmembrane proteins) and are chemically amphiphatic, whereby the transmembrane domain of the integral membrane protein consists of nonpolar amino acid residues that form an $\alpha$-helix. The fact that the transmembrane domain contains nonpolar amino acid residues means that it is hydrophobic and, thus, forms strong interactions with the tails of membrane lipids. In turn, it stands to reason that the intracellular and extracellular domains of the integral membrane proteins are hydrophilic. Unlike integral proteins, peripheral proteins are located along the intracellular surface of the lipid bilayer. Peripheral proteins are bound to the cell membrane by hydrogen bonds or electrostatic interactions with the hydrophilic portions of membrane lipids or integral proteins. 


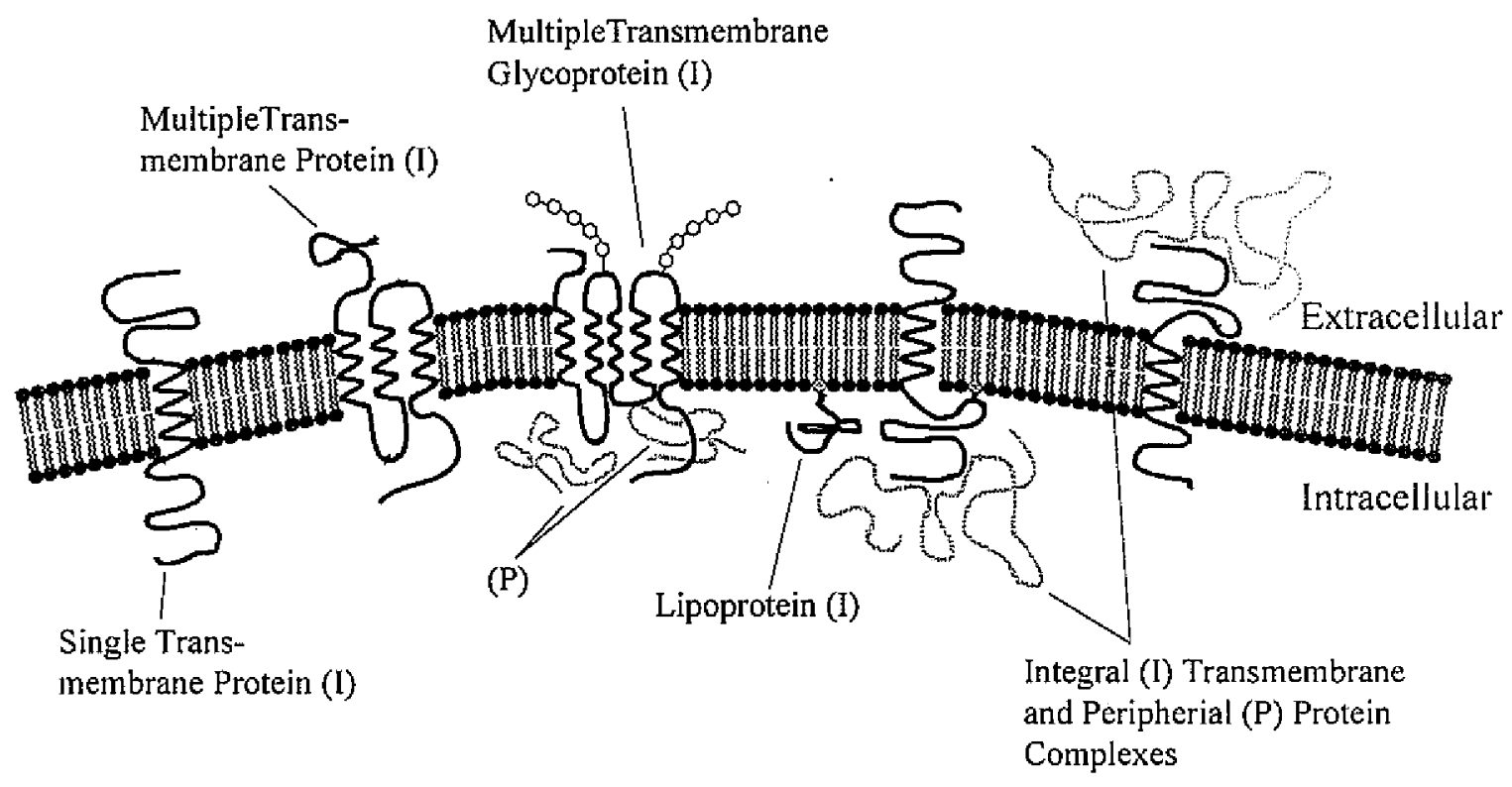

Figure 2.2.2 A schematic representation of the association between integral (I) and peripheral $(\mathrm{P})$ membrane proteins and the cell membrane. This figure was adapted from Ref. [35]. 
In view of the fluidic nature of a cell membrane, one would suspect that perhaps the molecules of the cell membrane are homogeneously distributed throughout the membrane. However, the cell membrane is in fact asymmetric in that the inner and outer membrane surfaces have different molecular components and are associated with different physiological activities. For instance, glycolipids and glycoproteins (proteins containing sugar moieties, e.g. ion channels) are only found on the extracellular half of the plasma membrane. These glycosylated molecules form the majority of the sugar coating around a cell known as the glycocalyx. The presence of sugars on the extracellular membrane region is thought to provide intercellular recognition between the cells in a tissue and provides identifying markers to cells of the immune system. This contrasts phospholipids, which play no part in intercellular recognition, and can be found on both the extracellular and intracellular halves of the cell membrane. Though in general, phospholipids are found predominantly on either the intra-, or extracellular halves of the membrane bilayer, e.g., phosphatidylserine being found mainly in the intracellular region of the plasma membrane of red blood cells [36]. Membrane-associated proteins must also be oriented in a specific way and delivered to a particular membrane region to ensure proper function of the protein and, subsequently, the cell.

\subsubsection{Electrical Properties of Membranes}

All cell-associated membranes have electrical properties which are defined by the membrane structure, composition, and function. This gives rise to an overall electrical potential of the cell membranes. This potential can be thought of as having two primary components: (a) a transmembrane potential, usually referred to simply as the membrane 
potential, and (b) surface potentials (charges). Generally, the electrical properties of the cell membrane play a major role in the physiological processes of the cell [37]. The cell membrane potential and the surface charge density show distinct variations in diseased cells, such as cystic fibrosis cells [38], and cancer cells $[37,39,40]$. In the following discussion, a brief description of the aforementioned contributions to the electrical potential of a cell membrane is given as it pertains to the plasma membrane. Though, these principles can, to an extent, be extrapolated to the electrical properties of organelle membranes, e.g., the mitochondrial membrane, as well.

The transmembrane potential (hereafter referred to as the membrane potential) arises as a result of the differences in electrical charge between the intracellular and extracellular membrane regions. This difference in electrical charge can be created through either electrogenic pumping or passive diffusion of inorganic ions in or out of the cell. The latter case is thought to be the dominant process occurring in animal cells and typically involves diffusion of the ions $\mathrm{K}^{+}, \mathrm{Na}^{+}, \mathrm{Ca}^{+}$, and $\mathrm{Cl}^{-}$through ion-selective channels across the cell membrane. The driving force behind the flow of ions through a membrane channel is the electrochemical gradient present across the membrane. This electrochemical gradient is comprised of an ion concentration gradient and a voltage gradient of these ions actoss the cell membrane, whereby one force drives the ions through the channel while the other force opposes the passage of ions. When these two forces are equally balanced, the result is no net flow of ions through the channel and the electrochemical gradient for the ion is zero. The voltage gradient (i.e., the membrane potential) under this equilibrium condition is an equilibrium potential commonly referred to as a resting potential. The resting potential can be calculated by the Nernst equation, 


$$
\mathrm{V}=-(\mathrm{RT} / \mathrm{zF}) \ln \left(\frac{\mathrm{C}_{\text {out }}}{\mathrm{C}_{\text {in }}}\right) \cong-60 \mathrm{mV} \log \left(\frac{\mathrm{C}_{\text {out }}}{\mathrm{C}_{\text {in }}}\right)
$$

where $\mathrm{V}$ is the resting potential in volts, $\mathrm{R}$ is the gas constant, $\mathrm{T}$ is the temperature in $\mathrm{K}, \mathrm{z}$ is the charge on the diffusible ion, $\mathrm{F}$ is Faraday's constant $\left(2.3 \times 10^{4} \mathrm{cal} / \mathrm{V} \cdot \mathrm{mol}\right)$, and $\mathrm{C}_{\text {out }}$ and $\mathrm{C}_{\mathrm{in}}$ are the ion concentrations on the outside and inside of the cell membrane, respectively. Although several inorganic ions have concentration gradients allowing passage across the cell membrane, the membrane potential in animal cells is thought to be affected predominantly by the $\mathrm{K}^{+}$leak channel present in those membranes (through which $\mathrm{K}^{+}$ions diffuse freely in and out of the cell). Alternative explanations to the view of passive diffusion of $\mathrm{K}^{+}$being the main cause of the resting potential have been proposed, but have not been widely accepted $[41,42]$. Measured values for the resting potential of these cells range anywhere from $-20 \mathrm{mV}$ to $-200 \mathrm{mV}$, with typical values in the area of $-70 \mathrm{mV}$. The negative sign for the resting potential reflects the relative concentration of ions on either side of the membrane. It should also be noted that changes in membrane permeability due to membrane excitation result in an action potential. This idea is best illustrated by the change in membrane permeability of a neuron upon excitation.

The other primary component of the electrical potential of a cell membrane is surface charges. These charges are the result of an asymmetric distribution of the molecules (i.e., membrane proteins and lipids) that comprise the membrane. These molecules give rise to surface charges as a consequence of charged groups on the molecules. Under the most common physiological conditions in natural environments, cells tend to be negatively charged due to the charges of the molecules found in the plane of the membrane and the glycocalyx. Both positive and negative charges found in the membrane plane come about 
from the hydrophilic regions of membrane proteins and phospholipids. Surface charges from phospholipids are mostly the result of the negatively charged hydrophilic head of phosphatidylserine, which as a -1 charge. This membrane lipid is maintained on the inner layer of the membrane bilayer by the ATP-driven pump aminophospholipid translocase. The glycocalyx is a coating of the outer membrane of the cell formed by the sugar moieties of glycoproteins and glycolipids. Portions of the sugar moieties distal to the protein or lipid contain neuraminic acids, which at a physiologically desirable $\mathrm{pH}$, are dissociated (i.e., they are negatively charged). It is most common for the glycocalyx to contribute a preponderance of the negative charge found on the extracellular side of the plasma membrane.

\subsection{Mitochondrial Membrane Potential}

The bioenergetic behavior of mitochondria in the intact cell is governed by: (1) the supply of substrate from the cytoplasm, (2) the turnover of ATP by cytoplasmic and plasma membrane energy-requiring processes, (3) the ionic environment of the cytoplasm, particularly in relation to $\mathrm{Ca}^{2+}$, and (4) the redox state of the cell [43]. Variations in each of these parameters can be observed depending on the energy demand of the cell, particularly in excitable cells such as those in muscle and brain. The bioenergetics of in situ mitochondria can be influenced by a wide variety of cellular stimuli, including plasma ion channel activation, hormonal signaling, and oxidative stress. For instance, the oxidative stress can be achieved through the generation and/or inefficient detoxification of reactive oxygen and nitrogen species such as superoxide, hydrogen peroxide, hydroxyl ions, nitric oxide or peroxynitrite. Since all of these parameters are dependent on the mitochondrial membrane 
potential $\left(\Delta \Psi_{m}\right.$, as opposed to the plasma membrane potential $\left.\left(\Delta \Psi_{p}\right)\right)$, one needs to consider how to monitor/determine this component.

Measuring the membrane potential of isolated mitochondria is a relatively simple process in that substrates, inhibitors, and ionophores can be added to the preparation with confidence. Whereas intact cells require greater care to ensure that an agent has access only to the mitochondrion and does not create interference in other parts of the cell. The plasma membrane of most cells is generally only sufficiently permeable to glucose and pyruvatelactate so as to inhibit the entry of undesired substances. Access by substrates such as succinate or adenine nucleotides usually indicates that the plasma membrane has been permeabilized. Not all mitochondrial inhibitors can permeate across the plasma membrane. For instance, atractyloside cannot access the adenine nucleotide translocator (ANT) in intact cells, whereas bongkrekic acid appears to permeate. Protonophores cannot be used in the same way as with isolated mitochondria: firstly, they permeate all membranes, affecting the transplasma membrane $\Delta \mathrm{pH}$ and short-circuiting other membranes with $\mathrm{H}^{+}$-translocating ATPases (lysosomes, synaptic vesicles, chromaffin granules, etc.). It is important to note that the addition of protonophores dramatically depletes cytoplasmic ATP, not only by preventing oxidative phosphorylation, but also by allowing the ATP synthase to reverse and hydrolyse glycolytically generated ATP. The latter can be prevented by using a combination of protonophore and oligomycin, as long as glycolysis is kinetically sufficient to supply the cell's ATP.

Potassium uniport ionophores such as valinomycin hyperpolarize the plasma membrane by clamping the plasma membrane potential, $\Delta \Psi_{\mathrm{p}}$, close to the $\mathrm{K}^{+}$diffusion potential, though a side-effect of this is that the mitochondria are then depolarized since the 
cytoplasm and matrix each contain $\sim 100 \mathrm{mM} \mathrm{K}^{+}$. The $\mathrm{K}^{+} / \mathrm{H}^{+}$exchanger nigericin, on the other hand, can be used in cells to decrease mitochondrial $\Delta \mathrm{pH}$ and hyperpolarize the inner

membrane. It should be noted that the compartmentation induced by these processes within the cell means that measurements of ATP/ADP ratios, glutathione reduction state, etc. do not accurately reflect values in the matrix as a whole. However, one approach that is underutilized in whole-cell bioenergetics, and that is accessible as long as cells can be obtained in suspension, employs an oxygen electrode. This approach can obtain more precise information on the bioenergetic status of the in situ mitochondria than can fluorescence approaches for the purpose of monitoring $\Delta \Psi_{\mathrm{m}}$.

\subsubsection{Monitoring in situ Mitochondrial Membrane Potential}

Virtually all techniques for monitoring $\Delta \Psi$ across the inner mitochondrial membrane utilize membrane-permeant cations, which distribute themselves across the membrane according to their Nernst equilibria. The challenge at the cellular level is how to estimate the concentration gradient between mitochondrial matrix and cytoplasm. Since the probes permeate across the lipid bilayer, rather than through specific transport proteins, they show no membrane selectivity. If allowed to equilibrate with a concentration $\left[\mathrm{C}^{+}\right]$out in the medium, the concentrations of probe in the cytoplasm and matrix will be respectively:

$$
\begin{aligned}
{\left[\mathrm{C}^{+}\right]_{\text {cylo }} } & =\left[\mathrm{C}^{+}\right]_{\text {oul }} \times 10^{\left(\Delta \Psi_{\mathrm{p}} / 60\right)} \\
{\left[\mathrm{C}^{+}\right]_{\text {martix }} } & =\left[\mathrm{C}^{+}\right]_{\text {cyio }} \times 10^{\left(\Delta \varphi_{\mathrm{II}} / 60\right)} .
\end{aligned}
$$

Combining these equations results in:

$$
\left[\mathrm{C}^{+}\right]_{\text {marrix }}=\left[\mathrm{C}^{+}\right]_{\text {out }} \times 10^{\left(\Delta \Psi_{\mathrm{p}}+\Delta \Psi_{\mathrm{in}} / 60\right)} .
$$


Note that, when equilibrated with a fixed extracellular concentration of probe, the concentration in the matrix will be equally affected by changes in $\Delta \Psi_{m}$ and $\Delta \Psi_{p}$. The constant (60) in the exponents of these equations came from the fact that $2.3 \mathrm{RT} / \mathrm{zF}=\sim 60 \mathrm{mV}$ at room temperature, as seen in Eq. (2.2).

Studies with isolated mitochondria in suspension often make deliberate use of fluorescent dye aggregation and consequent quenching, which occurs above a critical concentration in the matrix; thus the total fluorescence of the mitochondrial suspension in a cuvette will change as probe is accumulated and quenched in the matrix. This allows changes in matrix accumulation to be monitored continuously without the need to separate mitochondria from the incubation medium. On the other hand, studies with intact cells may be performed under loading conditions, which are either above or below the threshold at which matrix quenching occurs. The three techniques most commonly used are flow cytometry, confocal imaging at single mitochondrial resolution and non-confocal imaging at single-cell resolution. The first two must be performed in non-quenched mode, while with single-cell imaging either mode may be used.

\subsection{2. $\Delta \Psi_{\mathrm{m}}$ Monitoring in Non-quench Mode}

The threshold concentration required for aggregation of most fluorescent membrane potential probes is in the range of $1-100 \mu \mathrm{M}$, and must be determined empirically. With cells in susupension this is most easily done by decreasing the external probe's concentration until addition of protonophore no longer produces an increase in total fluorescence. With cells attached to coverslips and imaged by digital fluorescence microscopy, probe loading can be decreased until protonophore addition no longer produces a transient increase in 
single-cell fluorescence due to the release of probe into the cytoplasm from the quenched matrix.

The non-quench mode is used in flow cytometry, where the fluorescence of single cells is determined as they pass through the excitation beam. Flow cytometry is used when one wishes to compare $\Delta \Psi_{\mathrm{m}}$ in two populations of cells, or to determine the heterogeneity of potentials in a population in which, for instance, some cells are undergoing apoptosis [44]. The fluorescence will be proportional to the total probe accumulated within the matrix of the cell, and will of course be responsive to changes in either the plasma or the mitochondrial membrane potential. It is important not to exceed the quench threshold at which the mitochondrial fluorescence ceases to be proportional to the accumulated dye. For the commonly used probe $\mathrm{DiOC}_{6}(3)$, this means loading cells with no more than $1 \mathrm{nM}$. Studies have revealed that excessive concentrations of this probe (up to $40 \mathrm{nM}$ ) render the whole-cell signal almost totally insensitive to changes in $\Delta \Psi_{\mathrm{m}}$, while sensitivity to $\Delta \Psi_{\mathrm{p}}$ is retained [45].

Non-quench mode is also utilized when single mitochondria, or clusters of mitochondria, are to be imaged by either confocal microscopy or high-resolution nonconfocal microscopy focusing on cells with thin membrane processes, such as neurons. The ratio of fluorescence intensity between the mitochondria and adjacent mitochondria-free cytoplasm can in theory be put into the aforementioned Nernst equation to derive $\Delta \Psi_{\mathrm{m}}$. Though in practice, the resolution of the confocal microscope is insufficient to image just the mitochondrial matrix, and the actual intensity within the matrix is diluted by the inclusion of cristae thus blurring the image. Nonetheless, qualitative real-time changes in $\Delta \Psi_{\mathrm{m}}$ in individual mitochondria can be detected by this technique. 


\subsection{3. $\Delta \Psi_{\mathrm{m}}$ Monitoring in Quench Mode}

As discussed in Section 2.3.1, the redistribution of a membrane potential probe across the inner membrane of isolated mitochondria in response to changes in $\Delta \Psi_{\mathrm{m}}$ can be monitored in a cuvette without resolving or separating the individual organelles, only if the probe is loaded at a concentration sufficient to cause matrix quenching. An analogous approach can be taken with the mitochondria inside a single cell. Using a low resolution, non-confocal digital imager capable of measuring the total fluorescence from all parts of the cell, there is no need to resolve the individual mitochondria. The additional complication, however, is that the cell plasma membrane now acting as the cuvette, is of course permeant by the probe. Two biophysical principles govern the signal obtained under these circumstances. The first is that the probe is non-selectively permeant across both plasma and mitochondrial inner membranes and it seeks to achieve a Nernst equilibrium across both (Equation 2.3). However, because of the vastly greater surface to volume ratio of the inner membrane/matrix compared with that of the plasma membrane/cytoplasm, the probe reequilibrates much faster between the matrix and the cytoplasm than between the cytoplasm and any external medium. The second is that the probe appears to retain its full fluorescence efficiency in the matrix until the quench threshold is attained, while the excess is nonfluorescent.

The uncertainties in the parameters required to precisely quantify $\Delta \Psi_{m}$ in cells are such that it is virtually impossible to arrive at a value for $\Delta \Psi_{\mathrm{m}}$ which is sufficiently reliable to be of use in thermodynamic calculations. However, one thing which can be done with considerable accuracy, due to the logarithmic nature of the Nernst equation, is to detect small changes in $\Delta \Psi_{\mathrm{m}}$, particularly while in quench mode. This is measured when a mitochondrial 
depolarization leads to an increase in whole-cell fluorescence, following release of probe from the quenched matrix to the unquenched cytoplasm. It is quite easy to detect a rapid change in the range of $3-5 \mathrm{mV}$ in $\Delta \Psi_{\mathrm{m}}$, for instance, as a consequence of shifts in the proton circuit. One powerful application of this approach is to assess whether mitochondria, under conditions of stress, retain the ability to generate ATP [46].

\subsection{Membrane Potential Probes}

Optical probes used in conjunction with imaging techniques allow variations of membrane potential to be mapped along neurons, within tissues or among cell populations. Probes can be divided into two categories based on their response mechanisms. Fastresponse probes operate via a direct effect of surrounding electric field on the electronic structure of fluorophores and are therefore capable of detecting millisecond transient potential changes in excitable cells [47]. Slow-response probes operate via potentialdependent changes in their transmembrane distribution (a molecular level response as opposed to electronic level for fast probes) and are suitable for detecting changes in average membrane potentials of non-excitable cells caused by respiratory activity, ion channel permeability, uncoupler/hyperpolarizer drugs, and other factors.

The magnitude of the potential-dependent fluorescence change generated by fast potentiometric dyes is usually small; $\sim 2-10 \%$ per $100 \mathrm{mV}$ is considered to be a good response [48]. However, one technique based on fluorescence resonance energy transfer between a potential-sensitive oxonol in the membrane interior and a surface-bound fluorescent lectin has produced millisecond fluorescence signal changes as high as $34 \%$ per $100 \mathrm{mV}[49]$. 
The methyl and ethyl esters of tetramethylrhodamine (TMRM and TMRE) have been the preferred dyes for the determination of membrane potential by quantitative imaging [50,51]. Determination of membrane potential by the application of the Nernst equilibrium requires that the transmembrane distribution of the dye depends only on the membrane potential, and that other processes such as dye aggregation and potential-independent interactions with intracellular components contribute minimally. TMRM and TMRE fulfill these requirements in several respects [52,53]. They are readily membrane permeant, and their strong fluorescence means that they can be used at low concentrations, thus avoiding aggregation. As their fluorescence is relatively insensitive to environment, spatially resolved fluorescence signals of TMRM and TMRE present an unbiased profile of their transmembrane distribution that can be related directly to the membrane potential via the Nernst equation [51,54].

The large negative inner-membrane potential of mitochondria is the basis for the selective staining of this organelle by a variety of cationic probes, including rhodamine 123 [55] and CMXRos [56] (see Appendix B for the detailed characterization of the CMXRos dye). Other cationic probes, including nonyl Acridine Orange and MitoFluor Green, stain mitochondria via a potential-independent mechanism, possibly involving interaction with the anionic phospholipid cardiolipin, which is abundant in the inner mitochondrial membrane [57].

\subsubsection{Autofluorescence}

Autofluorescence refers to fluorescence from endogeneous biomolecules of unstained cells. In mammalian cells, autoflurorescence comes from flavin coenzymes (flavin 
mononucleotide, flavin adenine dinucleotide and riboflavin) $\left[58^{*}\right]$, reduced pyridine nucleotides (nicotinamide adenine dinucleotide (NADH) and nicotinamide adenine dinucleotide phosphate (NADPH)) [59], aromatic amino acids (phenylalanine, tryptophan, and tyrosine) and nucleic acids (adenine, thymine, guanine, cytosine, and uracil) [60]. The absorption and emission maxima $\left(\lambda_{\mathrm{abs}} / \lambda_{\mathrm{em}}\right)$ measured at $\mathrm{pH} 7.4$ for flavins and pyridine nucleotides are $450 \mathrm{~nm} / 515 \mathrm{~nm}$ and $340 \mathrm{~nm} / 460 \mathrm{~nm}$, respectively. Flavins and pyridine nucleotides are necessary for cellular metabolism [61] and amino acids and nucleic acids are essential components of proteins and DNA/RNA. In contrast, phenol red $\left(\lambda_{\mathrm{abs}}=560 \mathrm{~nm} / \lambda_{\mathrm{em}}=578 \mathrm{~nm}[35]\right)$, which is commonly used as a $\mathrm{pH}$ indicator in cell culture media, is an unnecessary component for cell viability. Thus, removal of these essential biomolecules from cells would be toxic whereas culture media without phenol red would not affect cell morphology.

Autofluorescence becomes problematic when the level of fluorescent dye present in a sample is low enough such that the autofluorescent signal competes with that of the probe. Traditional methods for overcoming autofluorescent signals, mainly for flow cytometric applications, are quite elaborate and can be difficult to implement. However, within the past couple of decades much simpler methods have been devised to overcome this problem [6265]. Many new dyes are now commercially available that absorb and emit in the same spectral region as the autofluorescent molecules, but are designed to "out-fluoresce" these endogenous chromophores when bound to the intended cellular target sites, even at low concentrations [66]. This approach is sufficient for microscopic and flow cytometric applications, but not particularly useful for hole burning applications. For instance, the aforementioned $\mathrm{pH}$ indicator phenol red will hole burn. This poses a problem in that a probe 
molecule whose absorption band overlaps with the phenol red absorption band will result in competing hole burning processes, and in turn, present difficult to interpret results.

Fortunately, the absorption bands of autofluorescent molecules and phenol red are typically measured at wavelengths less than $600 \mathrm{~nm}$. Therefore, dye molecules excited at longer wavelengths $(>600 \mathrm{~nm})$ typically result in lower levels of background autofluorescence [67] as well as decreased photochemically induced damage to the cell [68]. Furthermore, at longer wavelengths, light scattering by dense media is greatly reduced, resulting in deeper penetration of excitation light into thick tissue sample. A cationic lipophilic dye known as rhodamine 800 (MF680) is useful in this regard because its absorption maximum occurs near $680 \mathrm{~nm}$ which is well removed from that of autofluorescencing species. The next section gives a more extensive description of the behavior of MF680.

\subsubsection{Characterization of MF680}

MF680 is a carbocationic lipophilic dye that locates itself preferentially in the inner and outer membranes of mitochondria due to its lipid-enriched composition and lowered membrane potential relative to extramitochondrial areas. The molecular structure and absorption spectrum of MF680 in 99:1 (v/v) water/DMSO at room temperature are described in figures 2.4.1 (a) and (b), respectively. The absorption and emission experiments of MF680 in various organic solvents as well as in plasma and in mitochondrial suspensions have been extensively performed in Refs. [69-71]. During these room temperature studies, Sakanoue et al. [71] discovered some unique features of this probe. The absorption peaks as well as the emission peaks of MF680 in isolated mitochondria shift depending on the valinomycininduced potassium diffusion potential, which led them to suggest a linear relationship 
(a)

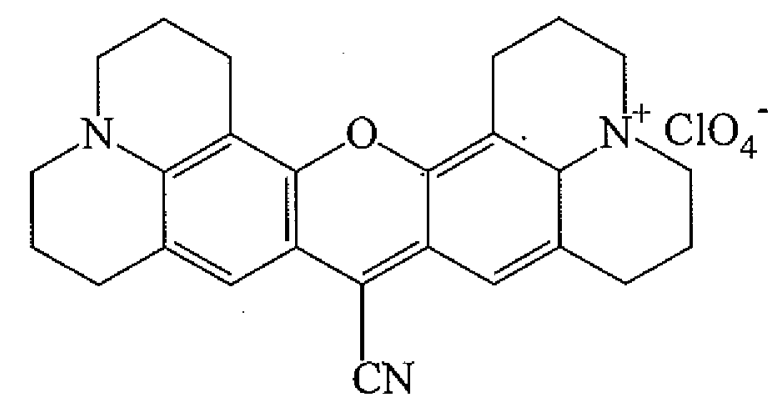

(b)

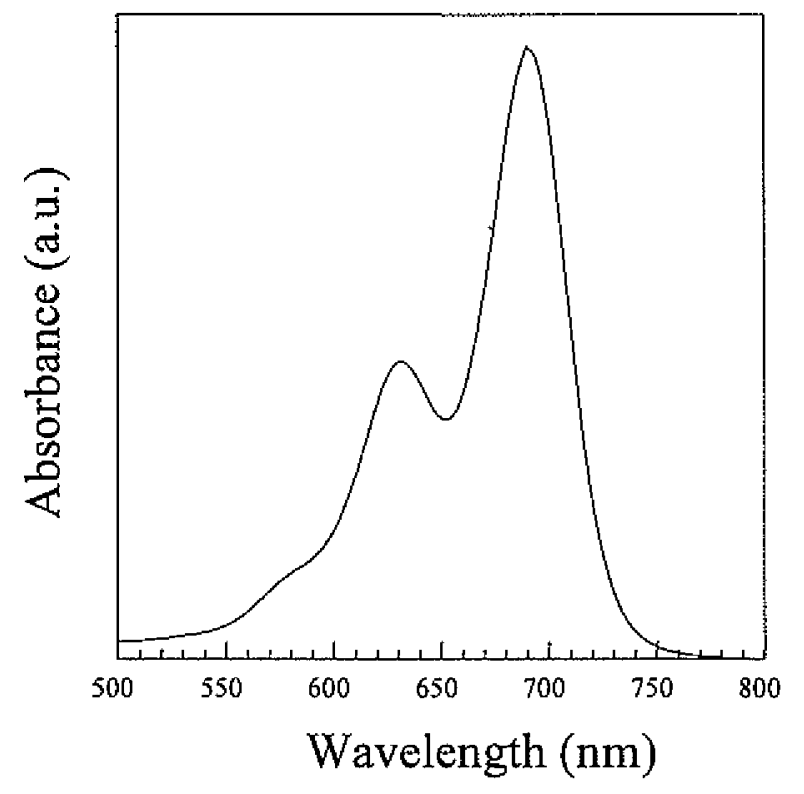

Figure 2.4.1 (a) Molecular structure of rhodamine 800 (MF680) and (b) a room temperature absorption spectrum of MF680 in 99:1 (v/v) water/DMSO, measured using Varian Cary 100 UV-VIS spectrometer. The absorption maxima are measured at $632 \mathrm{~nm}$ and $691 \mathrm{~nm}$. 
between the optical responses of MF680 (in the absorption difference at 730 minus $685 \mathrm{~nm}$ ) and $\Delta \Psi_{m}[71]$.

Dye uptake studies revealed the optimum MF680 concentration to be $15 \mu \mathrm{M}$ in order to achieve the highest uptake/free dye ratio in the energized mitochondria in suspension [70]. Although, in this thesis study, $250 \mathrm{nM}$ was empirically chosen to stain the tissue samples in order to preserve organelle-specificity of the dye, to avoid entrapment of free unbound dye in intercellular matrices, and of course to minimize the cytotoxicity. Confocal microscopy images of MF680 in cryomicrotomically sliced $(\sim 30 \mu \mathrm{m})$ cancerous tissue sample (same type of tissue as one utilized for studies presented in Chapter 5) resembled the images of the OV 167 and VOSE14 cell lines presented in Ref. [5]. By conducting the sodium azide / BODIPY tests described in Ref. [5], the site-specificity of the dye in the tissue samples was confirmed. Moreover, these images ascertained that the majority of the signals did indeed come from the intracellular matrices (supposedly mitochondria), and that the free dye molecules were not trapped in the intercellular matrices, thereby confirming $250 \mathrm{nM}$ to be an appropriate concentration employed for the hole burning imaging experiments.

Fluorescence excitation spectra (at 5.2 K) of MF680 in cellular matrices, in comparison to the fluorescence excitation of the dye in hyperquenched glassy water ( $\mathrm{HGW}$ ), are presented in Figure 2.4.2. A noteworthy feature of the spectra for these cell lines is the red-shifted peak positions of the dye depending on the magnitude of the mitochondrial membrane potentials. See Appendix B for the mitochondrial membrane potential estimation of the model cell lines. The mean fluorescence lifetime of MF680 was found to be $1.8 \mathrm{~ns}$ in aqueous solution at $77 \mathrm{~K}[5]$ and $1.90 \mathrm{~ns}$ in plasma at room temperature [70]. This slight increase in lifetime is supposedly attributable to the binding of MF680 to the plasma moieties 


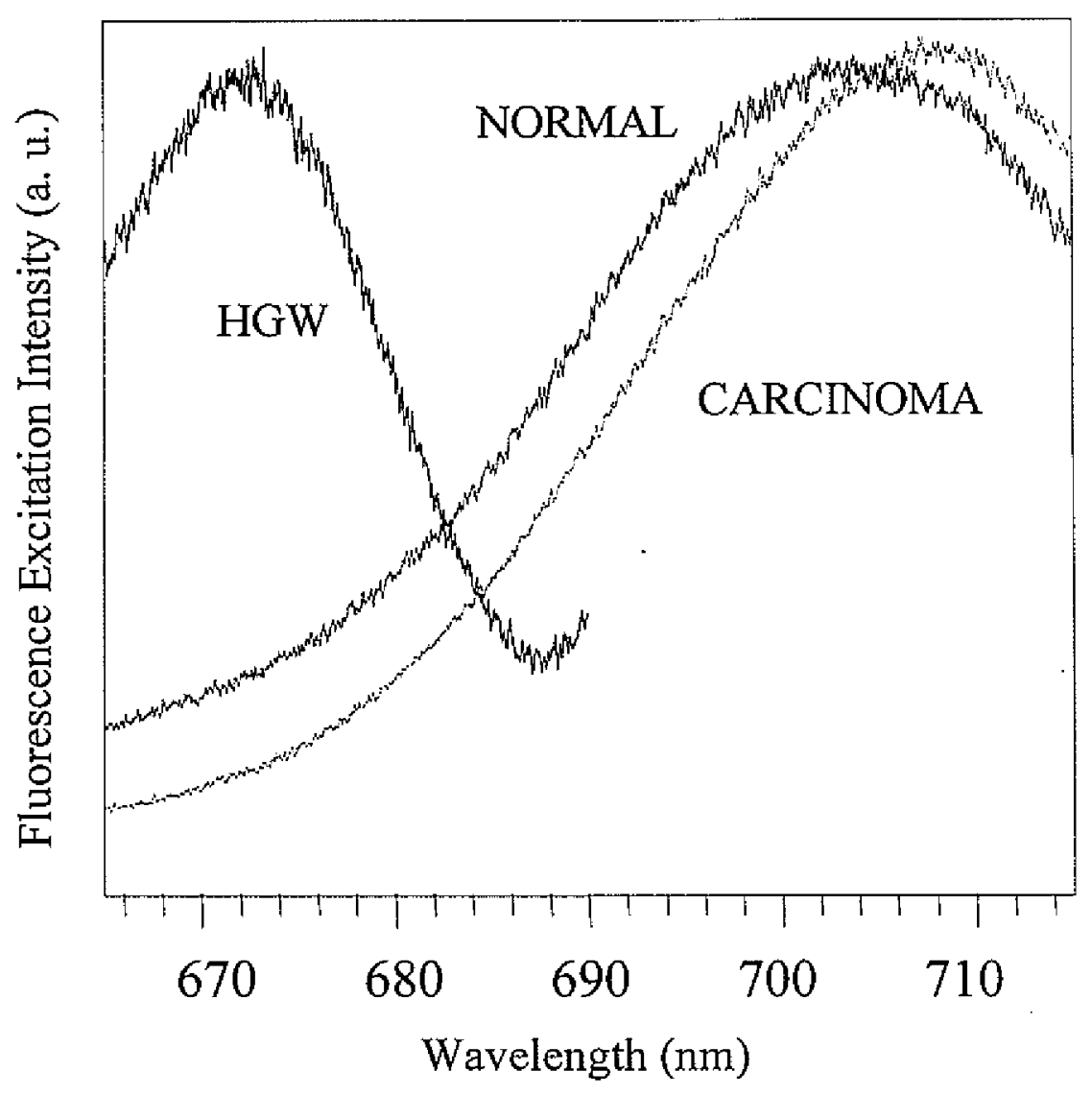

Figure 2.4.2 Fluorescence excitation spectra, measured at 5.2 K, of MF680 in carcinoma (OV167) and analogous normal (VOSE14) cellular matrices, and in hyperquenched glassy water (HGW). Note that the ratio of differences in mitochondrial membrane potentials of these cell lines, obtained with flow cytometry (See Appendix B), was about 1.5 (unitless) with OV167 furnishing the higher potential. This figure was adapted from Ref. [4]. 
and to albumin as well as the presence of other proteins such as globulin and lipoproteins. The fluorescence lifetime of $1.8 \mathrm{~ns}$ was taken for hole growth kinetics analysis as presented in Chapter 4 and 5. Detailed hole burning characteristics of MF680 in mitochondria will be described in Section 3.1.

\section{References:}

1. Mitchell, P. Science, 1979, 206, 1148.

2. Chen, L. B. Annu. Rev. Cell Biol., 1988, 4, 155.

3. Ehrenberg, B., Montana, V., Wei, M.-D., Wuskell, J. P., Loew, L. M. Biophys. J., $1988,53,785$.

4. Walsh, R. J., Reinot, T., Hayes, J. M., Kalli, K. R., Hartmann, L. C., Small, G. J. J. Lumin., 2002, 98, 115.

5. Walsh, R. J., Reinot, T., Hayes, J. M., Kalli, K. R., Hartmann, L. C., Small, G. J. Biophys. J., 2003, 84, 1299.

6. Lehninger, A. L. The Mitochondrion: Molecular Basis of Structure and Function, 1965, W. A. Benjamin, New York.

7. Benda, C. Arch. Anat. Physiol., 1898, 393.

8. Sjorstrand, F. S. J. Cell. Comp. Physiol., 1953, 42, 15.

9. Sjorstrand, F. S. Nature, 1953, 171, 30.

10. Palade, G. E. Anat. Rec., 1952, 114, 427.

11. Palade, G. E. J. Histochem. Cytochem., 1953, 1, 188.

12. Palade, G. E. in Enzymes: Units of Biological Structure and Function (Gaebler, O. H. ed.), 1956, Academic Press, New York, pp. 185.

13. Tyler, D. The Mitochondrion in Health and Disease, 1992, VCH Publishers, Inc., New York.

14. Scheffler, I. E. Mitochondria, 1999, Wiley-Liss, Inc., New York. 
15. Chen, L. B., Rivers, E. N. in Genes and Cancer (Carney, D., Sikora, K., eds.), 1990, John Wiley and Sons, Inc., New York, pp: 127.

16. Summerhayes, I. C., Lampidis, T. J., Bernal, S. D., Nadakavukaren, J; J., Nadakavukaren, K. K., Shepherd, E. L., Chen, L. B. Proc. Natl. Acad. Sci. USA, 1982, 79, 5292 .

17. Papa, S., Capuano, F., Capitanio, N., Lorusso, M., Galeotti, T. Cancer Res., 1983, 43., 834 .

18. Nadakavukaren, K. K., Nadakavukaren, J. J., Chen, L. B. Cancer Res., 1985, 45, 6093.

19. Ara, G., Aprille, J. R., Malis, C. D., Kane, S. B., Cincotta, L., Foley, J., Bonventre, J. V., Oseroff, A. R. Cancer Res., 1987, 47, 6580.

20. Christman, J. E., Miller, D. S., Coward, P., Smith, L. H., Teng, N. N. Gynecol. Oncol., 1990, 39, 72 .

21. Modica-Napolitano, J. S., Aprille, J. R. Adv. Drug Deliv. Rev., 2001, 49, 63.

22. Mitchell, P. Biol. Rev., 1966, 41, 445.

23. Mitchell, P. Nature, 1961, 191, 423.

24. Bereiter-Hahn, J., Voth, M. Microscopy Research and Technique, 1994, 27, 198.

25. Yaffe, M. P. Science, 1999, 283, 1493.

26. Wallace, D. C. Science, 1999, 283, 1482.

27. Bereiter-Hahn, J. in International Review of Cytology / A Survey of Cell Biology (Vol. 122; Jeon, K. W., Friedlander, M., eds.), 1990, Academic Press, San Diego, pp.1.

28. Trimmer, P. A., Swerdlow, R. H., Parks, J. K., Keeney, P., Bennett, J. P. Jr., Miller, S. W., Davis, R. E., Parker, W. D. Jr. Exp. Neurol., 2000, 162, 37.

29. Djaldetti, M. Acta Haemat., 1982, 68, 241.

30. Eapen, C. E., Madesh, M., Balasubramanian, K. A., Pulimood, A., Mathan, M., Ramakrishna, B. S. Scand. J. Gastroenterol, 1998, 33, 975.

31. Steim, J. M., Tourtellotte, M. E., Reinert, J. C., McElhaney, R. N., Rader, R. L. Proc. Natl. Acad. Sci. USA, 1969, 63, 104. 
32. Weislander, A., Ulmius, J., Lindblom, G., Fontell, K. $B B A, 1978,512,241$.

33. Schmidt, C. F., Barenholz, Y., Thompson, T. E. Bioochemistry, 1977, 16, 2649.

34. Yeagle, P. L. The Membranes of Cells ( $2^{\text {nd }}$ edn.), 1993, Academic Press, San Diego, pp. 18-42.

35. Milanovich, N. Ph.D. Dissertation, 1999, Iowa State University.

36. Huang, C. Klin. Wochemschrift, 1990, 68, 149.

37. Glaser, R. in Biophysics of the Cell Surface (Glaser, R., Gingell, D., eds.), 1990, Springer-Verlag, New York, pp. 173.

38. Ackerman, M. A., Clapman, D. E. New Eng. J. Med., 1997, 336, 1575.

39. Petty, H. R. Molecular Biology of Membranes: Structure and Function, 1993, Plenum Press, New York, pp. 353.

40. Monen, S. H., Schmidt, P. H., Wondergem, R. J. Membrane Biol., 1998, 161, 247.

41. Bashford, C. L., Pasternak, C. A. J. Membrane Biol., 1984, 79, 275.

42. Bashford, C. L., Pasternak, C. A. TIBS, 1986, 11, 113.

43. Nicholis, D. G., Ward, M. W. Trends Neurosci, 2000, 23, 166.

44. Mathur, A., Hong, Y., Kemp, B. K., Barrientos, A. A., Erusalimsky, J. D. Cardiovascular Research, 2000, 46, 126.

45. Mather, M., Rottenberg, H. Biochem. Biophys. Res. Commun, 2000, 273, 603.

46. Ward, M. W., Rego, A. C., Frenguelli, B. G., Nicholls, D. G. J. Neurosci, 2000, 20 . 7208 .

47. Loew, L. M. Pure Appl. Chem., 1996, 68, 1405.

48. Johnson, I. Histochem. J., 1998, 30, 123.

49. González, J. E., Tsien, R. Y. Biophys. J., 1995, 69, 1272.

50. Gross, D., Loew, L. M. Methods Cell Biol., 1989, 30, 193.

51. Loew, L. M. Methods Cell Biol., 1993, 38, 195. 
52. Ehrenberg, B., Montana, V., Wei, M.-D., Wuskell, J. P., Loew, L. M. Biophys. J., 1988, 53, 785 .

53. Farkas, D. L., Wei, M.-D., Febbroriello, P., Carson, J. H., Loew, L. M. Biochemistry, $1989,56,1053$.

54. Loew, L. M. in Cell Biology: A Laboratory Handbook $\left(2^{\text {nd }}\right.$ edn.; Celis, J. E. ed.), 1998, Academic Press, San Diego, pp. 375-379..

55. Chen, L. B. Methods Cell Biol., 1989, 29, 103.

56. Poot, M, Zhang, Y.-Z., Krämer, J. A., Wells, K. S., Jones, L. J., Hanzel, D. K., Lugade, A. G., Singer, V. L., Haugland, R. P. J. Histochem. Cytochem., 1996, 44, 1363.

57. Rashid, F., Horobin, R. W. Histochemistry, 1990, 94, 303.

58. Benson, R. C., Meyer, R. A., Zaruba, M. E., McKhann, G. M. J. Histochem. Cytochem., 1979, 27, 44 .

59. Aubin, J. E. J. Histochem. Cytochem., 1979, 27, 36.

60. Cantor, C. R., Schimmel, P. R. Biophysical Chemistry, Part II: Techniques for the Study of Biological Strucutre and Function, 1980, W. H. Freeman and Co., New York, pp. 377.

61. Alberts, B., Bray, D., Lewis, J., Raff, M., Roberts, K., Watson, J. D. Molecular Biology of Cell ( $3^{\text {rd }}$ edn.), 1994, Garland Publishing, New York, pp. 610.

62. Hirschfeld, T. J. Histochem. Cytochem., 1979, 27, 96,

63. Roederer, M, Murphy, R. F. Cytometry, 1986, 7, 558 .

64. Steinkamp, J. A., Stewart, C. C. Cytometry, 1986, 7, 566.

65. Corsetti, J. P., Sotirchos, S. V., Cox, C., Cowles, J. W, Leary, J. F., Blumberg, N. Cytometry, 1988, 9, 539 .

66. Haugland, R. P., Handbook of Fluorescent Probes and Research Chemicals $\left(9^{\text {th }}\right.$ edn.), 2002, Molecular Probes, Inc., Eugene.

67. Beumer, T. L., Veenstra, G. J. C., Hage, W. J., Destrée, O. H. J. Trends Genet., 1995, 11,9 . 
68. Bloom, J. A., Webb, W. W. J. Histochem. Cytochem., 1984, 32, 608.

69. Raue, R., Harnisch, H. Heterocycles, 1984, 21, 167.

70. Abugo, O. O., Nair, R., Lakowicz, J. R. Anal. Biochem., 2000, 279, 142.

71. Sakanoue, J., Ichikawa, K., Nomura, Y., Tamura, M. J. Biochem., 1997, 121, 29. 


\section{CHAPTER 3. INTRODUCTION TO HOLE BURNING IMAGING}

All tissues are comprised primarily of a similar set of components, these being a combination of lipids, proteins (functional/connective), and nucleic acids. The various combinations of these components are what define many different cell types, which in turn allow us to organize and classify the tissues they comprise. Cancer is an anomalous type in that characteristics prevalent in normal, functioning tissue become distorted as cells designated for tumorigenicity begin to proliferate extremely rapidly, resulting in deviation from normal structural controls and order. A diagnostic system based upon observing this distortion, would be a great benefit to pinpointing these anomalous cell lines, depending on the scale of the detection, with single cell structural anomaly detection being the ultimate in sensitivity. The basis for such a diagnostic system is found within the molecular probing technique of hole burning imaging (HBI).

\subsection{Introduction to Nonphotochemical Hole Burning}

Persistent spectral hole burning is a site selective spectroscopic technique developed to probe the inhomogeneously broadened absorption spectra of impurities (guests) in crystalline and amorphous solids (hosts) at low temperatures [1,2]. The conditions mandatory to observe spectral holes are: (a) a persistent frequency change mechanism in the excited state of the chromophore and (b) the presence of an inhomogeneously broadened absorption band of impurity. The absorption bands of chromophores in amorphous solids such as organic glasses, polymers, and biological macromolecules exhibit large inhomogeneous broadening. Such broadening is the result of the chromophores distribution throughout different environments, i.e. interactions between the chromophore and the host 
molecules gives rise to a distribution of transition frequencies [3]. A schematic illustration of this is depicted in Figure 3.1.1. Typical inhomogeneous broadenings of electronic absorption bands $\left(\Gamma_{\mathrm{inh}}\right)$ are $\sim 100-300 \mathrm{~cm}^{-1}$. Each particular chromophore does carry a finite width, which is called its homogeneous width $(\gamma)$ and is determined by the association of the total dephasing time $\left(T_{2}\right)$ with the optical transition of the chromophore in its environment. The linewidth of the homogeneous broadening is given by

$$
\gamma\left(\mathrm{cm}^{-1}\right)=\frac{1}{\pi T_{2} c},
$$

where $T_{2}$, the total dephasing time, is defined [4] by

$$
\frac{1}{T_{2}}=\frac{1}{2 T_{1}}+\frac{1}{T_{2}^{*}},
$$

where $T_{1}$ is the excited state lifetime of the chromophore and $T_{2}^{*}$ is the pure dephasing time of the molecules. As seen in Eq. (3.2), the total optical dephasing time of a chromophore consists of two parts: (1) the excited state lifetime (which is usually weakly dependent on temperature) and (2) the pure dephasing time, which is due to interactions of the chromophore with the host matrix and, is therefore, temperature dependent. Temperature dependence of electronic dephasing/spectral diffusion will be briefly discussed in the following section.

Any information that may be gleaned about a specific subset of chromophores in a particular local environment is usually well-hidden behind other subsets of absorbers. The use of extremely narrow linewidth lasers enables selective excitation of a spectrally narrow subset of absorbers in order to circumvent the screening effect of inhomogeneous broadening. Currently, two main frequency domain classes of selective excitation techniques 
(a)

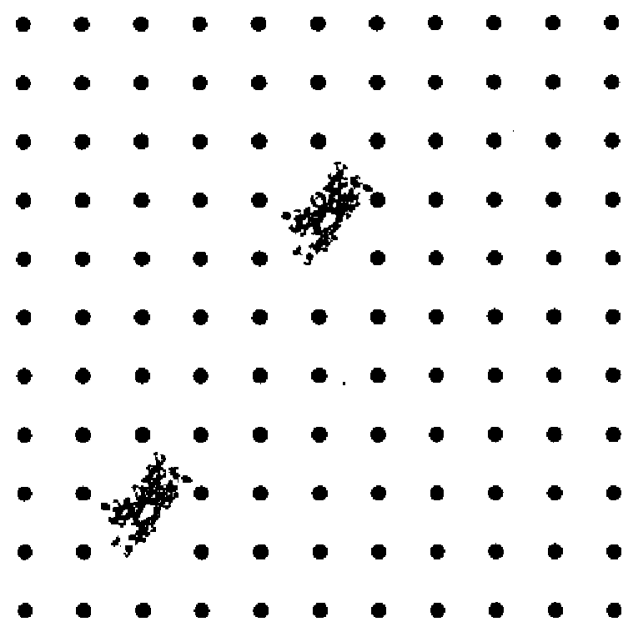

(b)

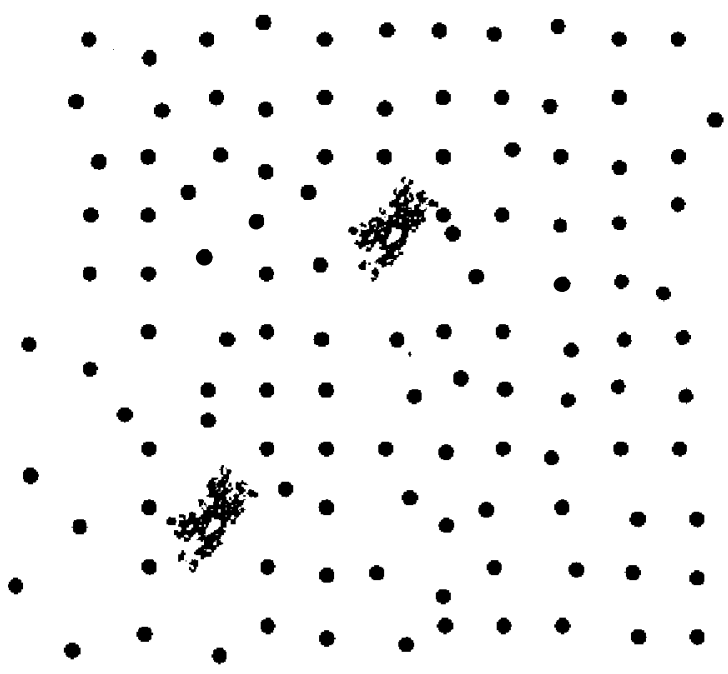

Figure 3.1.1 Diagrams (a) and (b) show schematic views of guest molecules in a perfect lattice (host) and in an amorphous lattice, respectively. In Part (a) the ZPLs appear at same transition frequency. However, in Part (b) the ZPLs appear at different frequencies, further described in Figure 3.1.2. 
exist: A. Fluorescence line narrowing spectroscopy (FLNS), which by its name indicates that fluorescence spectra obtained under selective excitation conditions can be much narrower than those obtained under non-selective excitation conditions. An in-depth description of the FLN mechanism is omitted here since the technique is out of scope of this dissertation; $\mathrm{B}$. Spectral hole burning spectroscopy. Hole burning is typically a two-part process consisting of a measurement of the absorption spectrum both before and after burning with a narrow frequency laser. Burning is the result of the irradiation of the amorphous system at the holeburning frequency $\omega_{\mathrm{B}}$. The hole is observed as a spectrally narrow area of reduced absorption in the absorption band close to $\omega_{\mathrm{B}}$.

One result of the selective excitation of specific chromophores is known as a zerophonon transition. A zero-phonon line (ZPL) is an electronic transition that does not involve a change in the total number of phonons. The ZPL is accompanied by a phonon wing, which is known as a phonon side-band (PSB). The PSB arises due to (linear) electron-phonon coupling between the initial and final electronic states of the chromophore and the phonon bath of the host matrix. The ZPL and PSB together form a single site absorption profile and it, at the low temperature limit using harmonic approximation, is given by $[5,6]$

$$
L(\Omega-v)=\prod_{j=1}^{N} \sum_{R=0}^{\infty} \frac{e^{-s_{j}} S_{j}{ }^{k}}{R !} l_{R}\left(\Omega-v-R \omega_{j}\right)
$$

where $v$ is a ZPL frequency, $l$ is the line shape function and $j$ corresponds to the discrete pseudolocalized or localized phonons and intramolecular vibrational modes. $S_{j}$ is the HuangRhys factor (which is a measure of the electron-phonon coupling strength), and $\omega_{j}$ is the center frequency of one-phonon profile with respect to the ZPL of the $j^{\text {th }}$ mode. For $R=0, L$ is the lineshape of the ZPL with a homogeneous width, $\gamma$. Values of $R(R=1,2,3 \ldots)$ 
correspond to one-, two-, three- ... phonon profiles, which for $\mathrm{R} \geq 1$, are given by the convolution of the one-phonon profile with itself $R$ times. The convolution of a single-site absorption profile with a Gaussian shaped site-distribution function (SDF), whose mean is at $v_{m}$, gives the inhomogeneously broadened absorption spectrum,

$$
A(\Omega)=\int d v N\left(v-v_{m}\right) L(\Omega-v)
$$

where $N\left(v-v_{m}\right)$ is the probability function of finding a site with a zero-phonon transition frequency at $\nu$. (The hole profile at burning time $\tau$ is then derived from $A_{\tau}(\Omega)-A_{0}(\Omega)$ where $A_{\tau}(\Omega)$ and $A_{0}(\Omega)$ stand for the absorption spectrum after and before burning, respectively.) Figure 3.1.2 schematically shows an inhomogeneously broadened absorption band and single site absorption profiles, each consisting of a Lorentzian-shaped ZPL and an accompanying PSB.

In a hole-burning experiment, the reduction of specific chromophores forms a zerophonon hole (ZPH) and an accompanying phonon-sideband hole (PSBH). The width of the ZPL can be obtained from the ZPH width, $\Gamma_{h}$, and it is given by

$$
\Gamma_{\mathbf{h}}=2 \gamma
$$

Note that the lasers applied in the experiments performed as a basis for this dissertation had a linewidth that was typically more than ten times narrower than $\Gamma_{\mathrm{b}}$. The width of the ZPH, which coincides with the burn frequency $\left(\omega_{B}\right)$, was utilized in determining the lifetimes of the excited states. According to hole burning theory [2], the Huang-Rhys factor, $S$, is obtainable from the integrated intensity of the real-PSBH divided by that of ZPH at $\lambda_{\mathcal{B}}$ (burning wavelength). However, with nonphotochemical hole burning, the blue-shifted antihole, usually from the pseudo-PSBH, interferes with the real-PSBH. $S$ can also be 


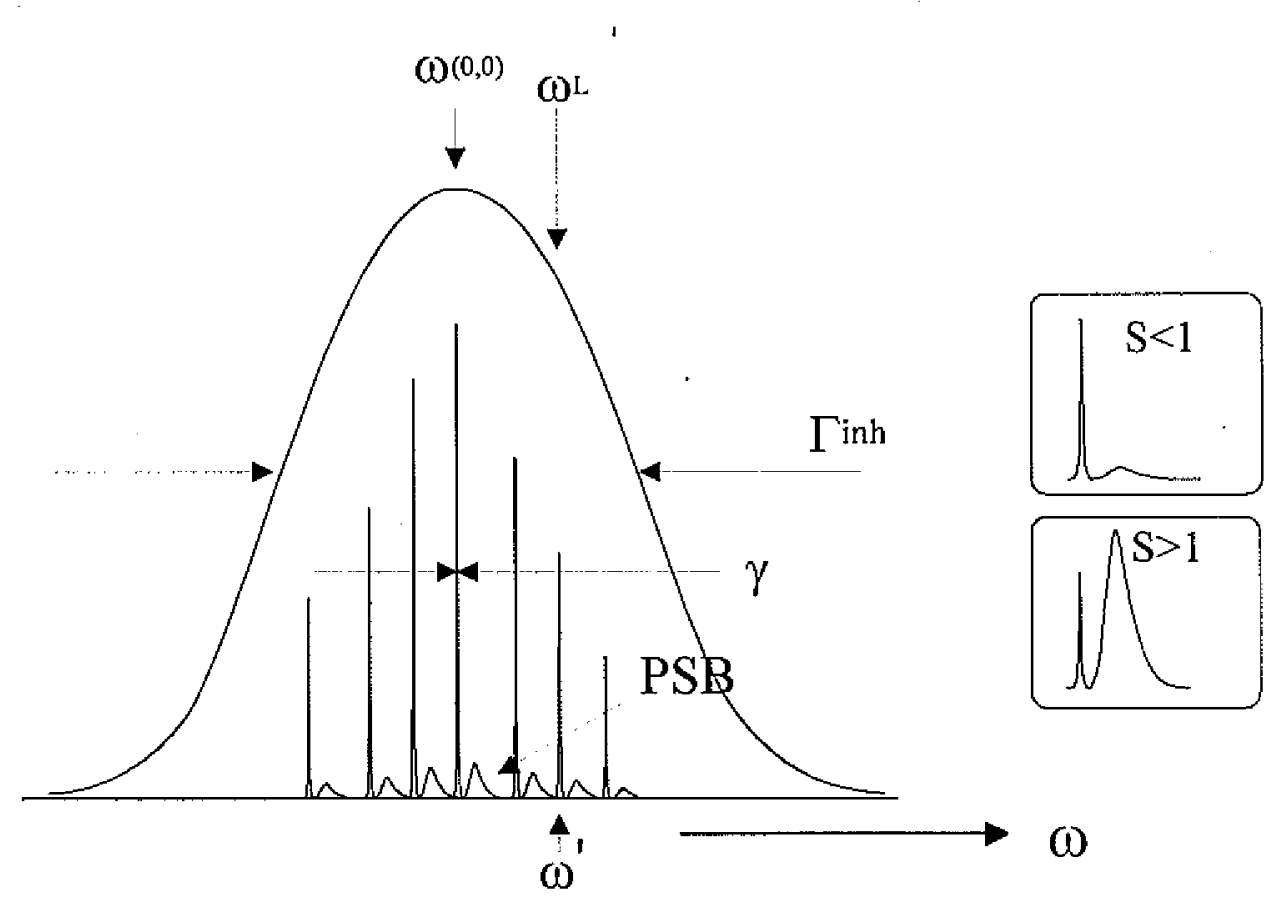

Figure 3.1.2 Diagram of homogeneously $(\gamma)$ and inhomogeneously $\left(\Gamma_{\text {inh }}\right)$ broadened absorption bands. The ZPLs and their phonon side bands are enlarged for clarification. $\omega_{\mathrm{L}}$ is the laser frequency for selective excitation of a narrow isochromat. The windows at the right describe the schematic difference of the relative ZPL/PSB structures with respect to Huang-Rhys factor (electronphonon coupling strength). Strong and weak couplings are defined by $\mathrm{S}>1$ and $\mathrm{S}<1$, respectively. 
determined considering the fact that the saturated fractional depth of the ZPH is equal to $\exp (-S)$, otherwise known as the Franck-Condon factor for the ZPL [7,8]. Normally, intense ZPLs are observed in weak electron-phonon coupling $(S<1)$ cases (see Figure 3.1.2). A typical ZPH-PSBH spectrum for a "deep" burn is shown in Figure 3.1.3. As seen in the figure, the hole spectra of biological probes in cellular matrices often display low-frequency vibrational modes, in addition to those characteristic of strong/weak electron-phonon coupling.

Spectral hole burning also provides valuable information about inhomogeneous broadening. In an action spectrum, the ZPHs are burned under constant burn fluency conditions across the absorption band of interest $[9,10]$. Assuming that the nonphotochemical hole burning efficiency is independent of the burn fluency, the packets of ZPHs serve to describe the inhomogeneously broadened absorption band.

Nonphotochemical ZPH action spectroscopy has often been used to probe states of weak absorption that contribute to an absorption band. Because of the inherent amorphous structural disorder of the chromophores, the action spectrum during hole burning reveals the extent of heterogeneity, which is responsible for site inhomogeneous broadening, $\Gamma_{\text {inh, }}$ of an individual absorption band.

Throughout the past decade, spectral hole burning has been not only utilized to characterize in vitro cellular matrices, but has also been applied to photosynthetic proteinpigment complexes [7-10] in order to determine, for instance, excitation energy transfer pathways in the light-harvesting complexes of photosynthetic bacteria [11]. More recently, hole-burning techniques combined with high-pressure and/or external electric (Stark) fields have been applied to those systems in order to determine the pressure shift rates and the 


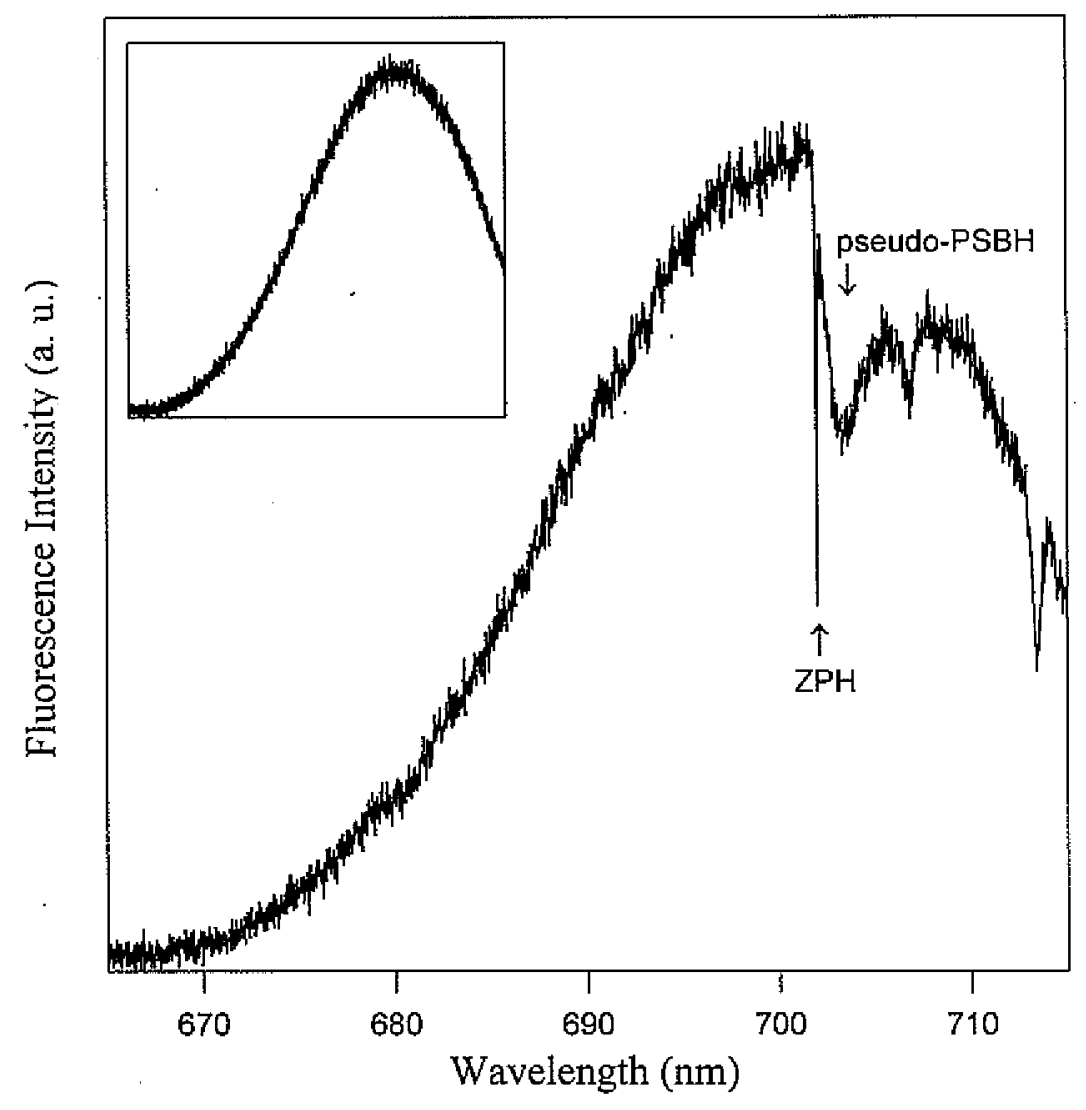

Figure 3.1.3 Fluorescence excitation pre- (inset) and post-burn spectra profiles for OV167 carcinoma cells incubated with MitoFluor Far Red 680. Note the formation of a zero phonon hole (ZPH) and the pseudo-phonon side band hole (PSBH) $30 \mathrm{~cm}^{-1}$ lower than the $\mathrm{ZPH}$. The two holes to the lower energy side of the PSBH are vibronic satellite holes due to vibrations of 95 and $227 \mathrm{~cm}^{-1}$. The figure was adapted from Ref. [12]. 
permanent dipole moment changes associated with the $S_{0} \rightarrow S_{l}\left(Q_{y}\right)$ state. The relation of the Stark fields to the spectral holes will be further discussed in Section 3.3.

\subsection{Hole Burning Mechanisms}

There are three mechanisms for hole burning spectroscopy [2]. All three have in common a process that leads to an absorption decrease at the frequency $\left(\omega_{\mathrm{B}}\right)$ of the absorbed photon. The three types of spectroscopies are referred to as photochemical hole burning (PHB), nonphotochemical hole burning (NPHB) and population bottleneck hole burning, which is not described in this section. PHB can be observed for both amorphous and crystalline hosts and it involves a photoreaction of the chromophore in its excited state, such as tautomerization, bond-breaking, and isomerization. PHB was first observed for free base phthalocyanine in n-octane, whereby selective electronic excitation resulted in an intramolecular proton tautomerization [13].

NPHB, on the other hand, does not require the absorbing chromophore to be photoreactive. Nonphotochemical processes result from a "permanent" change in the environment around the chromophore following completion of the optical excitation cycle. As the host configurations are required to be changed in the case of NPHB, NPHB is generally restricted to glass, polymer and protein hosts, with a few exceptions [14]. NPHB was first observed for perylene and for 9-aminoacridine in an ethanol glass [15]. In NPHB, laser excitation of a guest molecule results in a rearrangement of the microenvironment surrounding the guest molecule. A NPHB mechanism is favored in amorphous systems because of their inherent structural disorder which allows for reconfiguration of host-guest interactions. NPHB is a reversible process, i.e. the hole generated by this process can be 
filled, e.g. by an increase of temperature. The absorption of the photophysical product, known as an antihole, is formed within the original inhomogeneous absorption band. Hayes and Small [16], using a two-level system (TLS) model, first proposed a mechanism for NPHB in 1978. The schematic diagram is described in Figure 3.2.1. This model premised that amorphous systems consist of bistable host-guest configurations (TLS) that are described by a static distribution of TLS $[1,2,17]$. As depicted in Figure 3.2.1, excitation at the frequency $\omega_{\mathrm{B}}$ is followed by a tunneling process which favors this excited state over the ground state by a factor of $\sim 1000$, and finally relaxation to the ground state as depicted on the right-hand side of the diagram. The tunneling frequency $W=\omega_{0} \exp (-\lambda)$, where $\lambda$ is the tunnel parameter equals to $\mathrm{d}(2 \mathrm{~m} V)^{1 / 2} / \hbar, V$ is the barrier height determined by the guest-host configuration, $m$ is the effective mass of the tunneling entity and $\omega_{0}$ is the harmonic frequency. Since the TLSs are asymmetric double well potentials, the new absorption frequency will be different, leading to the absence of absorption at $\omega_{\mathrm{B}}$. In 1981 , Hayes et al. [18] suggested that two types of TLS are involved in NPHB. The extrinsic TLSs $\left(\mathrm{TLS}_{\mathrm{ex}}\right)$ were supposed to be strongly associated with the absorbing centers and are responsible for the initiation of the hole formation. The intrinsic TLSs $\left(\right.$ TLS $_{\text {int }}$ ) of the host are connected with the excess free volume of glasses and were proposed to be the cause of the optical dephasing $[14,16]$. The chromophore (guest) initiates the TLS $S_{\text {ext }}$ that are associated with the chromophore and its inner shell of solvent molecules. NPHB occurs primarily in amorphous matrices due to a rearrangement of the host environment, triggered by the electron-TLS $S_{\text {int }}$ coupling. It is the optical excitation of the chromophore that generates the phonon-assisted tunneling processes that lead to the hole formation. The rate-detemining step for hole formation is the excited state phonon-assisted tunneling of $\mathrm{TLS}_{\mathrm{ext}}$. 


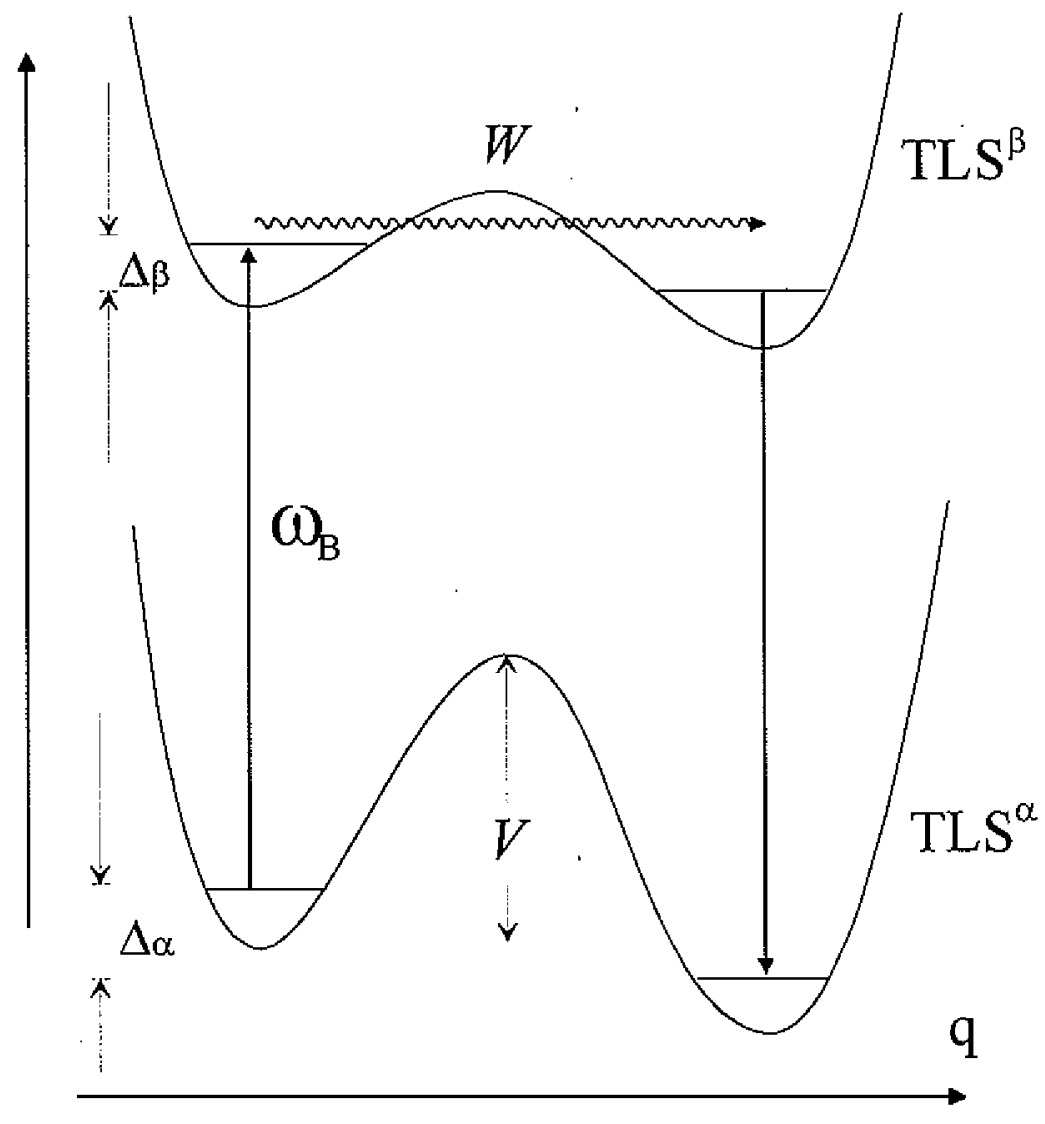

Figure 3.2.1 Diagram of TLSs coupled to a guest molecule in the ground state $(\alpha)$ and in the excited state $(\beta) . \Delta \alpha$ and $\Delta \beta$ are asymmetry parameters and $\mathrm{q}$ represents the intermolecular coordinate. $V$ is the magnitude of the energy barrier and $W$ $\left(=\omega_{0} \exp (-\lambda)\right)$ is the tunneling frequency. $\omega_{\mathrm{B}}$ stands for the excitation frequency. See text for details. 
Later on, it was proposed [19] that NPHB is the result of a hierarchy of configurational tunneling events that involve the faster relaxing $\mathrm{TLS}_{\mathrm{int}}$ which begin in the outer shell and terminate in the inner shell. This "outside-in" hierarchy of events causes the reduction of excess free volume in the outer shell (due to $T L S_{\text {int }}$ tunneling) and leads to an increase in the inner shell free volume about the chromophore. This process is illustrated as a cartoon in Figure 3.2.2 for the system of aluminum phthalocyanine tetrasulfonate (APT) in amorphous water. NPHB in the inner shell is then the result of prior relaxation events that allow for hole burning in the limit of $\mathrm{T} \rightarrow 0 \mathrm{~K}$. Moreover, the increase in the free volume for the probe in its inner shell of host molecules explains the blue-shifted antihole associated with ${ }^{1} \pi \pi^{*}$ states, since such states exhibit a red shift in going from the gas to the condensed phase.

Experimentally, spectral holes are measured in the fluorescence excitation mode or the transmission mode. Primarily, the fluorescence excitation method was utilized in the writing of this thesis. Detailed experimental setups can be found in the Experimental section of Chapter 4. The reader interested in the most recent NPHB mechanism involving multilevel extrinsic systems (MLS ext $_{\text {t }}$ should refer to the recent review by Reinot et al. [20].

\subsubsection{Dispersive Hole Growth Kinetics}

The working model of NPHB as a manifestation of tunneling between different glasschromophore configurations is currently widely accepted. The classification as dispersive stems from the mechanism: the amorphous matrix of a glass creates a distribution of phononassisted relaxation rates, $\mathrm{R}$, associated with $\mathrm{TLS}_{\mathrm{ext}}$, and cannot be described by a single rate constant. Hence, burn kinetics are dispersive. 


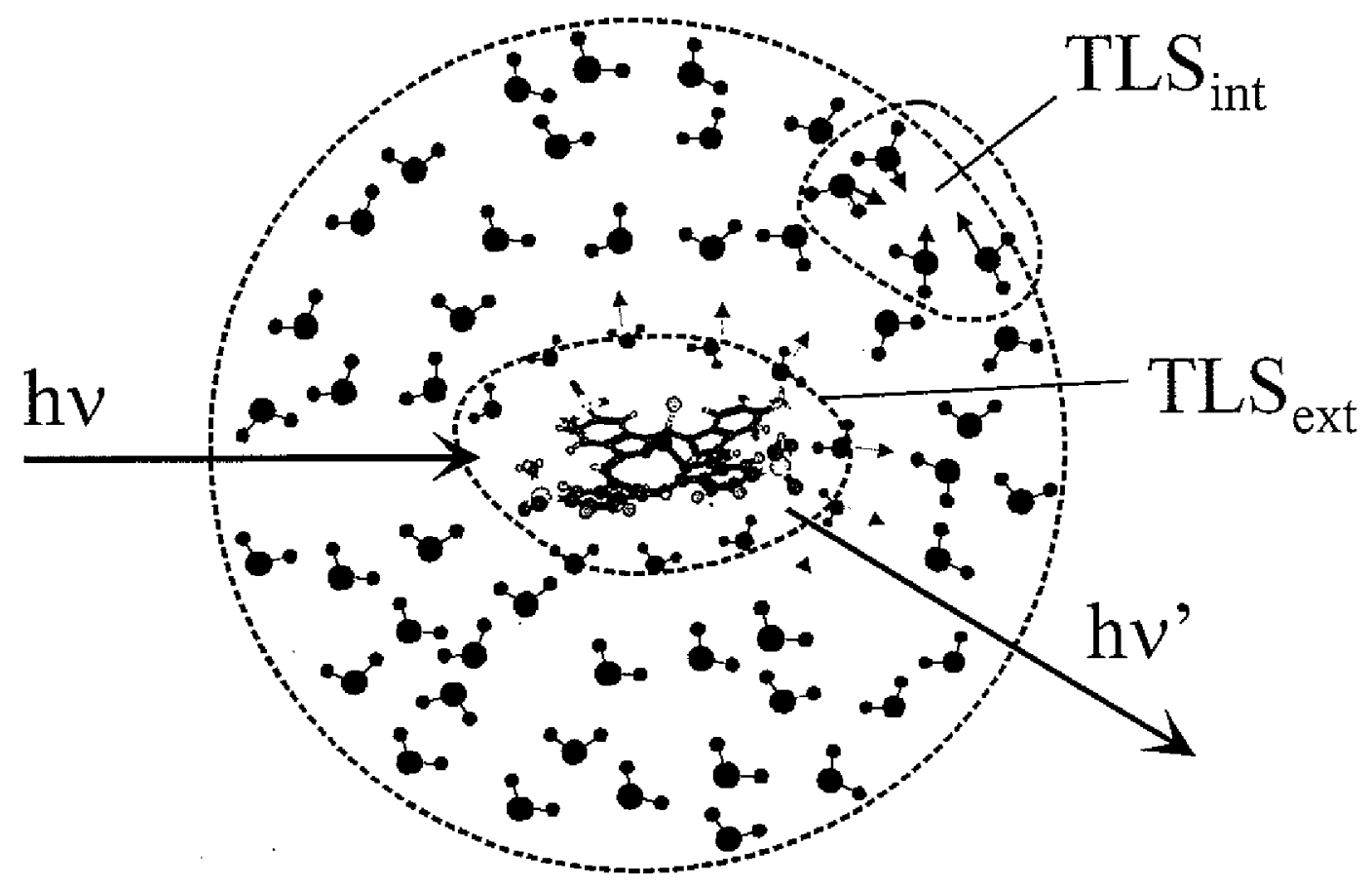

Figure 3.2.2 Schematic view of a NPHB mechanism. See text for discussion. The original figure was prepared by Dr. Tonu Reinot (Dept. of Chem., Iowa State University). 
Based on results for oxazine 720 in glycerol, polyvinyl alcohol thin films, and their deuterated analogues, Kenney et al. $[21,22]$ developed a theory to describe the dispersive hole growth kinetics of these systems using a Gaussian distribution function for the TLS $\mathrm{S}_{\mathrm{x} 1}$ tunneling parameter, $\lambda$, where

$$
g(\lambda)=N_{\lambda} \exp \left[\frac{-\left(\lambda-\lambda_{0}\right)^{2}}{2 \sigma_{2}{ }^{2}}\right]
$$

when $\lambda_{0}^{2} / 2 \sigma_{\lambda}{ }^{2} \gg>1$, and the normalization factor $N_{\lambda}=1 /\left(\sigma_{\lambda}(2 \pi)^{1 / 2}\right) . \lambda_{0}$ is the center of the Gaussian function, $g(\lambda)$, with a variance $\sigma_{\lambda}$. According to the theory, the square of the tunneling frequency $\mathrm{W}=\omega_{0} \exp (-\lambda)$ is proportional to the phonon-assisted tunneling relaxation rate, $R$. As a result the relaxation rate can be described as $R=\Omega_{0} \exp (-2 \lambda)$, where $\Omega_{0}$ is on the order of $\omega_{0}$, the harmonic frequency of TLS $S_{\mathrm{ext}}$ and is dependent on the TLS $\mathrm{S}_{\mathrm{ext}}$ deformation potential and the asymmetry parameter, $\Delta$. A distribution function for $\mathrm{R}$ can then be written based on Gaussian distribution functions for $\lambda$ as proposed by Jankowiak et al. [23-25] where the center of the Gaussian function is at $\lambda_{0}$ with a variance $\sigma_{\lambda}$. As a result of this approach, the fractional hole depth of the ZPH is $1-D(t)$, and $D(t)$ is defined as

$$
D(t)=(2 \pi)^{-1 / 2} \int_{-\infty}^{+\infty} d x \exp \left(\frac{-x^{2}}{2}\right) \exp \left(-\Sigma_{0} \xi(x) t\right)
$$

where $\Sigma_{0}=P \sigma \Omega_{0} \tau$ ( $P$ is the photon flux, $\sigma$ is the peak absorption cross section, and $\tau$ is the excited state lifetime), $x=\left(\lambda-\lambda_{0}\right) / \sigma_{\lambda}$, and $\xi(x)=\exp \left[-2\left(\lambda_{0}-\sigma_{\lambda} x\right)\right]$. For simplicity, the theory neglects the contribution of intrinsic dispersion from photoselection due to the polarization of the burn laser ( $\alpha$, the angle between the laser polarization and the transition dipole). Also neglected is the absorption of off-resonant laser excitation by the ZPL, $\omega$. Even so, the 
theoretical fit from this model consistently matched the experimental data and afforded determination of $\lambda_{0}$ and $\sigma_{\lambda}$.

More recently, the theory for dispersive hole growth kinetics of laser dyes in glasses was refined for the Al-phthalocyanine tetrasulfonate in a hyperquenched glassy water (APT/HGW) system [26], the result being

$$
D(\Omega, t)=\int d \omega L(\Omega-\omega) G(\omega) \int d \lambda f(\lambda) \int d \alpha \sin \alpha \cos ^{2} \alpha\left(e^{-P_{0 \rho}(\lambda) \mu(\omega \mathrm{B}-\omega)\left(\cos ^{2} \alpha\right.}\right),
$$

which is the fractional hole depth at frequency $\Omega$ following a burn for time $t$ with photon flux $P$, where $\omega$ is the ZPL frequency, $L(\Omega-\omega)$ is the single site absorption spectrum, $G(\omega)$ is the site excitation energy distribution function, $\sigma$ is the integrated absorption cross-section and $f(\lambda)$ is the normalized Gaussian distribution function for the $\operatorname{TLS}_{\mathrm{ext}}$ tunnel parameter, centered at $\lambda_{0}$. Although the probe chromophore used in Chapter 4 and 5 is different from that used in Ref. [26], a similar method was used to determine that $G(\omega)$ for the chromophore has a FWHM of $600 \mathrm{~cm}^{-1}$ (for $\mathrm{Q}_{\mathrm{y}}$ transition), with all burn frequencies located on the lower energy side of the absorption profiles obtained for the dye in vivo. Note here that the parameters $\lambda$ and $\alpha$ each define a distribution of hole burning rates that lead to dispersive kinetics, with the $\lambda$ distribution being the dominant factor [26] accounting for the first $80 \%$ of a burn. Because of this, Eq. (3.8) is typically represented in a simpler form as

$$
D\left(\omega_{\mathrm{B}}, t\right)=\int d \lambda f(\lambda) \int d \alpha \sin \alpha \cos ^{2} \alpha\left(e^{-\mu_{o f}(\lambda) \cos ^{2} \alpha t}\right)
$$

The NPHB quantum yield, $\phi(\lambda)$, is given by

$$
\phi(\lambda)=\frac{\Omega_{0} \exp (-2 \lambda)}{\Omega_{0} \exp (-2 \lambda)+\tau_{\mathrm{fl}}^{-1}}=\frac{\mathrm{R}_{\mathrm{HB}}}{\mathrm{R}_{\mathrm{HB}}+\mathrm{R}_{\text {relax }}} \approx \frac{\mathrm{R}_{\mathrm{HB}}}{\mathrm{R}_{\text {relax }}},
$$


where $\tau_{\mathrm{f}}$ is the fluorescence lifetime. For a detailed discussion, the reader should refer to Ref. [26,27]. It should be noted that, as a screening process for NPHB, spontaneous hole filling (SPHF) [28] and light-induced hole filling (LIHF) [29] must also be considered for more precise hole burning rate analysis.

The parameters obtained from fitting hole growth kinetics curves yield values for $\lambda_{0}$ and $\sigma_{\lambda}$, which, in essence, describe the relative disorder of an amorphous matrix ( $\lambda_{0}$ provides the mean distribution of tunneling rates while $\sigma_{\lambda}$ describes the variance of $\lambda$, i.e. the structural heterogeneity of the host matrix). The results of in vivo carcinoma cells and normal analogs based on mitochondrion specific staining [30] revealed that the cellular matrices of cancer cells could be viewed as being more structurally ordered than those of normal cells. Therefore, the parameters obtained from hole growth kinetics experiments, viz., $\lambda_{0}$ and $\sigma_{h}$, might be quantitatively used to distinguish between normal and cancerous cells and tissues.

\subsubsection{Temperature Dependence of Electronic Dephasing}

Aside from the above factors, the temperature dependence of electronic dephasing in glasses must also be considered. It is important to mention here that a wide variety of $T$. dependences, $\Delta \omega \propto T^{n}(1 \leq n \leq 2)$, have been observed [31-36] for a large number of impurity-amorphous host systems. This fact is exactly why a general theory to account for all observed T-dependences has not yet been obtainable. The reader is referred to Refs. $[37,38]$ for more complete discussions regarding the theoretical prediction of the $T$ dependence of dephasing. Briefly, the primary difference between the theories (besides the $\mathrm{TLS}_{\mathrm{int}}$-averaging procedure) is how the impurity-TLS $\mathrm{S}_{\mathrm{int}}$ coupling is treated. The choice of how this coupling is handled depends on whether one assumes that the dephasing of an 
impurity site is dominated, on average, by a single (or a small number of) nearby TLS int $_{\text {or by }}$ a "sea" of more distant and more weakly interacting TLS. These are referred to as offdiagonal modulation (ODM) (which allows for mixing of the tunnel state functions) and diagonal modulation (DM) (no mixing), respectively. ODM predicts a linear T-dependence in the high temperature limit independent of the nature of the impurity-TLS $\mathrm{Sint}_{\text {interaction, }}$ while the DM predicts power laws in the low temperature limit and does depend on the

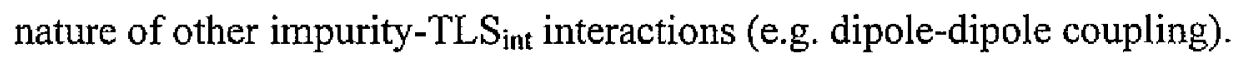

More recently, the Jackson-Silbey expression [39] stated the T-dependence of electronic dephasing for APT/HGW system as

$$
\Gamma-\Gamma_{0}=a \mathrm{~T}^{\alpha}+b_{1} \bar{n}\left(\omega_{1}\right)+b_{2} \bar{n}\left(\omega_{2}\right)+\cdots,
$$

where $\Gamma$ is half the measured holewidth and $\Gamma_{0}$ is the lifetime limited width of the ZPL. Though this expression is under constant scrutiny and is subject to further refinement. $\bar{n}=$ $[\exp (\hbar \omega / k \mathrm{~T})-1]^{-1}$ defines the phonon thermal occupation number. $a \mathrm{~T}^{\alpha}$ describes dephasing from the electron-TLS $\mathrm{S}_{\text {int }}$ coupling and the remaining two terms on the right-hand side of $\mathrm{Eq}$. (3.11) are from exchange coupling of pseudolocalized modes. For a variety of probe molecules in organic glasses, the values of $\alpha$ for the temperature range 0.1 to $\sim 10 \mathrm{~K}$ are $\approx 1.3$ $[1,4,40,41]$. These dephasing studies also characterize the quadratic electron-phonon coupling, whereas the aforementioned measurements of the frequencies and intensities of the phonons characterize the linear electron-phonon coupling. More recent results of the spectral diffusion in organic glasses are described in Refs. [42,43]. 


\subsection{Effects of External Electric (Stark) Fields}

Stark hole burning spectroscopy is now frequently applied in the study of probe molecules in various amorphous solids, such as polymers [44,45], proteins [46,47], and in vitro cellular systems $[30,48,49]$. In a Stark experiment, an external electric field (Stark field) causes the electronic transition frequency of a molecule to undergo a frequency shift, $\Delta \omega$, due to the interaction of the external field with the dipole moments and polarizabilities of the ground and excited electronic states of the probe. The induced frequency shift can be described as [50]

$$
\Delta \omega=\frac{1}{\hbar}\left[\left(\Delta \vec{\mu}_{0}+\Delta \vec{\alpha} \cdot \mathbf{E}_{\mathrm{int}}\right) \cdot f \mathrm{E}_{\mathrm{s}}+(1 / 2) \Delta \vec{\alpha} \cdot\left(f \mathbf{E}_{\mathrm{s}}\right)^{2}\right],
$$

where $\mathbf{E}_{\mathrm{S}}$ and $\mathbf{E}_{\text {int }}$ represent the Stark and internal electric fields experienced by the probe, respectively. $\Delta \vec{\mu}_{0}$ is the difference in the molecular dipole moment vectors of the ground and excited states, and $\hbar$ is Planck's constant. $f$ is the Lorentz local field correction factor where $f$ $=(\varepsilon+2) / 3$ and $\varepsilon$ is the dielectric constant of the matrix. Furthermore, $\Delta \vec{\alpha}$ is the difference in the polarizability tensors in the ground and excited electronic states and $\Delta \bar{\alpha} \cdot \mathbf{E}_{\mathrm{it \textrm {t }}}$ describes a matrix-induced change in the dipole, $\Delta \mu_{\text {ind }}$ contributing to the Stark shift. It should be noted that the first term in Eq. (3.12), $\left(\Delta \vec{\mu}_{0}+\Delta \bar{\alpha} \cdot \mathbf{E}_{\text {iur }}\right) \cdot f \mathbf{E}_{\mathrm{s}}$, describes the linear dependence of $\Delta \omega$ on an electric field while the second term represents the quadratic dependence. Since $\mathbf{E}_{\text {int }} \gg>$ $\mathbf{E}_{\mathrm{S}}$ for most of the molecular systems, a linear dependence of the frequency shift is typically observed in amorphous solids. Thus, the quadratic term in Eq. (3.12) can be neglected and the frequency shift is adequately described by

$$
\Delta \omega=\frac{1}{\hbar}\left[\left(\Delta \vec{\mu}_{0}+\Delta \vec{\alpha} \cdot \mathbf{E}_{\mathrm{int}}\right) \cdot f \mathrm{E}_{\mathrm{s}}\right]
$$


For hole burning systems, a linear Stark effect indicates that no shift in the center frequency of a ZPH will be observable after applying the Stark field. If, however, the systems are quadratically dependent on the Stark field, then the hole will red-shift relative to the hole position at zero field. A quadratic Stark effect will only occur when the external electric field is comparable in strength to the internal electric fields in the solid. Since typical hole burning systems undergo a linear Stark effect, including the one presented in this dissertation, the remainder of this discussion will pertain strictly to the linear behavior of amorphous systems in an external field.

Kador et al. later developed a relation between the hole width, $\Gamma_{\mathrm{h}}$, (FWHM, in $\left.\mathrm{cm}^{-1}\right)$ and the applied electric field [44], given as

$$
\Gamma_{\mathrm{h}}(\mathrm{F})=\gamma_{0}\left(1+\mathrm{F}^{2}\right)^{1 / 2},
$$

where $\gamma_{0}$ is the width of the $\mathrm{ZPH}$ at zero applied field $\left(\mathrm{E}_{S}\right)$, and

$$
\mathrm{F}=\frac{2 f \Delta \mu \mathrm{E}_{\mathrm{s}}}{h \gamma} .
$$

Here $\Delta \mu$ is the difference in magnitude between the ground $\left(\vec{\mu}_{\mathrm{g}}\right)$ and excited $\left(\vec{\mu}_{\mathrm{e}}\right)$ electronic state dipole moments, and $f$ is again the local field correction factor. Since the local field correction factors are usually not known, dipole moment changes are usually given as $f: \Delta \mu$ values. Note that the theory of Kador et al. holds even in the case of the hole saturation or power broadened, and therefore the experiment needs not necessarily be done in a shallow hole limit. A description of probe-matrix interactions can be developed by considering $\Delta \mu$ as having intrinsic molecular $\left(\Delta \mu_{0}\right)$ and matrix induced $\left(\Delta \mu_{\mathrm{ind}}\right)$ contributions [51] to the dipole moment change, i.e., $\Delta \vec{\mu}=\Delta \vec{\mu}_{0}+\Delta \vec{\mu}_{\text {ind }}$. The relative contribution to the overall dipole moment difference, $\Delta \vec{\mu}$, is dependent on the system. For probe molecules with an inversion 
center such as APT, $\left|\Delta \vec{\mu}_{0}\right|=0$ and thus, $\Delta \vec{\mu}=\Delta \vec{\mu}_{\text {ind }}$. Since the Stark broadening of holes that would occur is strictly matrix-induced, the absence of splitting observed in the preliminary cell study with MF680 [52] indicated that $\left|\Delta \bar{\mu}_{\text {ind }}\right|>>\left|\Delta \vec{\mu}_{0}\right|$ for MF680. Furthermore, $\Delta \vec{\mu}_{\mathrm{ind}}$ can be viewed as being composed of random $\left(\Delta \mu_{\mathrm{r}}\right)$ and nonrandom $\left(\Delta \mu_{\mathrm{rr}}\right)$ components [53] such that $\Delta \vec{\mu}_{\mathrm{ind}}=\Delta \vec{\mu}_{\mathrm{r}}+\Delta \vec{\mu}_{\mathrm{nr}}$, which will be referred to later in this section.

It also should be noted that, in the interpretation of an illustration of the Stark effect, the causes of the broadening or splitting of holes in an external applied electric field is based on the analysis of dipole moment interactions. Figure 3.3.1 provides a vector representation of the Stark effects for four different extremes of the angle $\theta$ between the transition dipole, $\mathbf{D}$, and the dipole moment difference, $\Delta \vec{\mu}_{0}$, either at $0^{\circ}$ or at $90^{\circ}$. In part (a) and (b) of the figure, when $\Delta \vec{\mu}_{0} \| \mathbf{D}$ (i.e. $\theta=0^{\circ}$ ), splitting of the hole is observed when the probe laser polarization, $\mathbf{E}_{\mathrm{L}}$, is oriented parallel to $\mathbf{E}_{\mathrm{S}}$, while hole broadening is observed when $\mathbf{E}_{\mathrm{L}}$ is oriented perpendicular to $\mathbf{E}_{\mathrm{S}}$. Similarly, part (c) and (d) represent the situations where $\Delta \vec{\mu}_{0} \perp$ D, where broadening and splitting are observed when $\mathbf{E}_{\mathrm{L}} \| \mathbf{E}_{\mathrm{S}}$ and $\mathbf{E}_{\mathrm{L}} \perp \mathbf{E}_{\mathrm{S}}$, respectively. If $\gamma$ is the angle between the transition dipole and the laser polarization, then the distribution about $\cos ^{2} \gamma$ of the ground and excited transition dipole moment around the laser polarization $\mathbf{E}_{\mathrm{L}}$ is represented by $\mathbf{D}$ vectors deviating about $30^{\circ}$ from the maxima of their angular distributions. As denoted by $\Delta \mu_{0}^{\mathrm{E}}$, the triangles represent the projections of the products of the dipole moment differences $\Delta \vec{\mu}_{0}$ and $\mathbf{E}_{\mathrm{S}}$. Note that, in contrast, when a matrix induced dipole moment change is caused by predominantly random electrostatic interactions with the matrix, i.e. $\left|\Delta \vec{\mu}_{r}\right| \gg>\left|\Delta \vec{\mu}_{\text {nr }}\right|$, only broadening is observed, irrespective of the angle between 
(a)

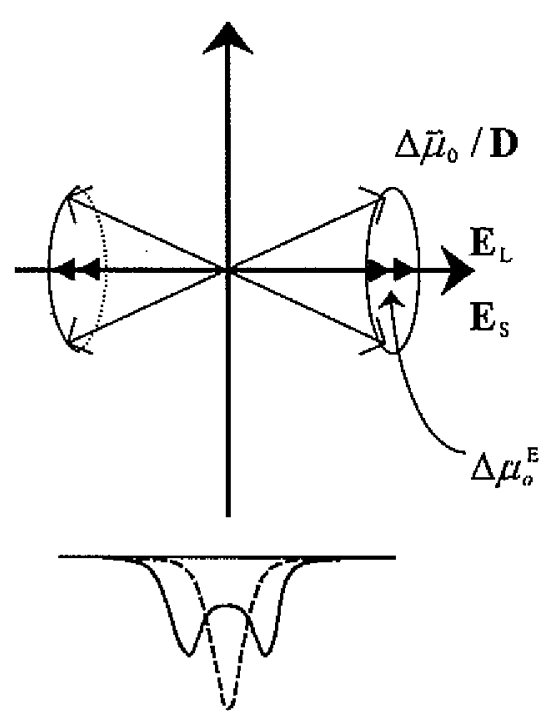

(c)
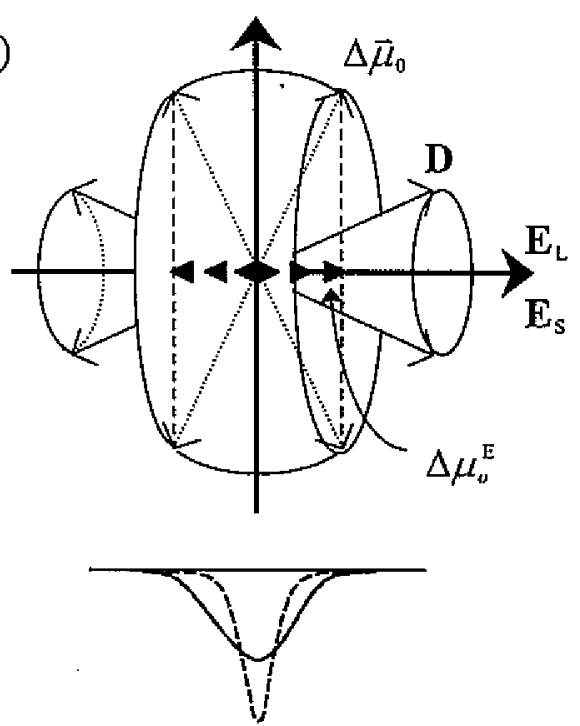

(b)

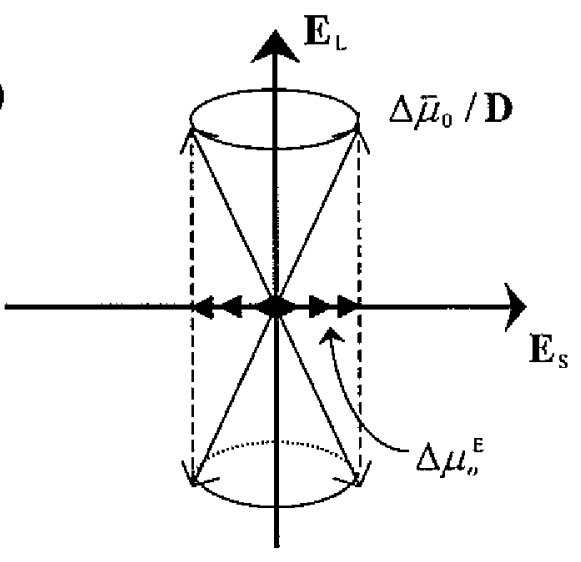

(d)
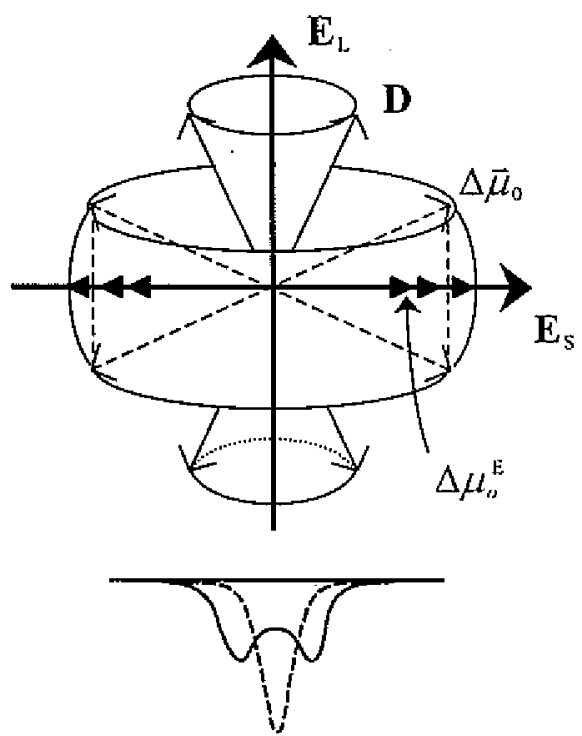

Figure 3.3.1 Vector representations of the Stark effects illustrating hole splitting and broadening. Conditions for each frame are as follows: (a) $\theta=0^{\circ}$ and $\mathbf{E}_{\mathrm{L}} \| \mathbf{E}_{\mathrm{S}}$ (b) $\theta=0^{\circ}$ and $\mathbf{E}_{\mathrm{L}} \perp \mathbf{E}_{\mathrm{S}}$ (c) $\theta=90^{\circ}$ and $\mathbf{E}_{\mathrm{L}} \| \mathbf{E}_{\mathrm{S}}$ (d) $\theta=90^{\circ}$ and $\mathbf{E}_{\mathrm{L} .} \perp \mathbf{E}_{\mathrm{S}}$. See text for further discussion. This figure was adapted from [54]. 
$\mathrm{E}_{\mathrm{L}}$ and $\mathrm{E}_{\mathrm{S}}$. Described in Chapter 4 is the case for MF680 in cancerous and normal tissues where broadening is observed when $\mathbf{E}_{\mathrm{L}} \| \mathbf{E}_{\mathrm{S}}$ and $\mathbf{E}_{\mathrm{L}} \perp \mathbf{E}_{\mathrm{S}}$. For MF680 in tissues, the difference in $\Delta \mu$ 's for parallel and perpendicular laser/electric field orientations is negligible, confirming $\left|\Delta \vec{\mu}_{\mathrm{r}}\right|>>\left|\Delta \vec{\mu}_{\mathrm{mr}}\right|$, which is consistent with the observation for the cell experiments $[30,52]$.

\subsection{Application of NPHB to Biological Anomaly Detection}

The preceding discussions have served the purpose of demonstrating the capacity of NPHB in discerning the differences in the structures of biological specimens. This has been presented as a thorough background for the research presented in Chapter 4 and 5. Based on the pioneering works of Milanovich et al. [48,49], a dye probe placed in a cell is expected to provide quantifiable NPHB (from hole growth kinetics studies) information regarding the assembly of that cell's matrix. In the present case, this will allow for a comparison between in vitro cancerous tissues and normal analogs based on specific staining of in vivo mitochondria, which have previously been discussed (see Section 2.4) and determined to be structurally anomalous for mitochondria. Moreover, the refined work of Walsh et al. [30] demonstrated that the NPHB technique combined with external electric (Stark) field is also a reliable approach, which should afford resolution of mitochondrial membrane potential differences between carcinoma and normal cell lines. Since then, it has been through continued refinements and molecular biological exploration that this technique has met with increasing success. By now, we have met with many ideological successes behind our objectives. The objective to find and target structural differences between normal and carcinoma tissues has been met with the discussion of mitochondrial anomalies; and the 
objective to present the ability of NPHB imaging for resolving and quantifying those structural differences has been covered in a fundamental sense.

\section{References:}

1. Hayes, J. M., Jankowiak, R., Small, G. J. in Topics in Current Physics, Persistent Spectral Hole Burning: Science and Applications (Moerner, W. E. ed.), 1987, Springer-Verlag, New York, pp. 153.

2. Jankowiak, R., Hayes, J. M., Small, G. J. Chem. Rev., 1993, 93, 1471.

3. Fridrich, J., Haarer, D. Angew. Chem. Int. Ed. Engl, 1984, 23, 113.

4. Völker, S. in Relaxation Processes in Molecular Excited States (Fünfschilling, J. ed.). 1989, Kluwer, Dordrecht, pp. 113.

5. Lyle, P. A. Ph.D. Dissertation, 1993, Iowa State University.

6. Lyle, P. A., Kolaczkowski, S. V., Small, G. J. J. Phys. Chem., 1993, 97, 6926.

7. Hayes, J. M., Gillie, J. K., Tang, D., Small, G. J. Biochim. Biophys. Acta, 1988, 932, 287.

8. Pieper, J., Voigt, J., Renger, G., Small G. J. Chem. Phys. Lett., 1999, $310,296$.

9. Reddy, N. R. S., Picorel, R., Small, G. J. J. Phys. Chem., 1992, 96, 6458.

10. Reddy, N. R. S., Cogdell, R. J., Zhao, L. Small, G. J. Photochem. Photobiol., 1993, 57,35 .

11. Matsuzaki, S., Zazubovich, V., Fraser, N. J., Cogdell, R. J., Small G. J. J. Phys. Chem. B, 2001, 105, 7049 .

12. Walsh, R. J. Ph.D. Dissertation, 2002, Iowa State University.

13. Gorokhovskii, A. A., Kaarli, R. K., Rebane, L. A. JETP Lett., 1974, $20,216$.

14. Jankowiak, R., Small, G. J. Science, 1987, 237, 618.

15. Kharlamov, B. M., Personov, R. I., Bykovskaya, L. A. Opt. Commun, 1974, 12, 191.

16. Hayes, J. M., Small, G. J. Chem. Phys., 1978, 27, 151. 
17. Reinot, T., Small, G. J. J. Chem. Phys., 2001, 114, 9150.

18. Hayes, J. M., Stout, R. P., Small, G. J. J. Phys. Chem., 1981, 74, 4266.

19. Shu, L., Small, G. J. Chem. Phys., 1990, 141, 447.

20. Reinot, T., Zazubovich, V., Hayes, J. M., Small, G. J. J. Phys. Chem. B, 2001, 105, 5083.

21. Kenney, M., Jankowiak, R., Small, G. J. Chem. Phys., 1990, 146, 47.

22. Kenney, M. Ph.D. Dissertation, 1990, Iowa State University.

23. Jankowiak, R., Rechert, R., Bässler, H. J. Phys. Chem., 1985, 89, 4569.

24. Jankowiak, R., Small, G. J. Phys. Rev. B, 1988, 37, 8407.

25. Jankowiak, R., Hayes, J. M., Small, G. J. Phys. Rev. B, 1988, 38, 2084.

26. Reinot, T, Small, G. J. J. Chem. Phys., 2000, 113, 10207.

27. Elschner, A., Bässler, H. Chem. Phys., 1988, 123, 305.

28. Shu, L, Small, G. J. J. Opt. Soc. Am. B, 1992, 9, 733.

29. Reinot, T., Hayes, J. M., Small, G. J. Chem. Phys., 1999, 110, 4820.

30. Walsh, R. J., Reinot, T., Hayes, J. M., Kalli, K. R., Hartmann, L. C., Small, G. J. Biophys. J., 2003, 84, 1299.

31. Hayes, J. M., Stout, R. P., Small, G. J. J. Phys. Chem., 1980, 73, 4129.

32. Reinecke, T. L. Sol. St. Commun., 1979, 32, 1103.

33. Lyo, S. K., Orbach, R. Phys. Rev. B, 1980, 22, 4223.

34. Lyo, S. K. Phys. Rev. Lett., 1982, 48, 688.

35. Huber, D. L, Broer, M. M., Golding, B. Phys. Rev. Lett., 1984, 52, 2281.

36. Reineker, P., Morawitz, H., Kassner, K. Phys. Rev., 1984, 29, 4546. 
37. Lyo, S. K. in Electronic Excitation and Interaction Processes in Organic Molecular Aggregates (Vol. 49; Reineker, P., Haken', H., Wolf, H. C., eds.), 1983, SpringerVerlag, New York.

38. Lyo, S. K., Orbach, R. Phys. Rev. B, 1984, 29, 2300.

39. Jackson, B., Silbey, R. J. Chem. Phys. Lett., 1983, 99, 331.

40. Silbey, R. J., Kassner, K. J. Lumin., 1987, 36, 283.

41. Jankowiak, R., Small, G. J. Chem. Phys. Lett., 1993, 207, 436.

42. Koedijk, J. M. A., Wannemacher, R., Silbey, R. J., Völker, S. J. Chem. Phys., 1996, $100,19945$.

43. den Hartong, F. T. H., van Papendrecht, C., Silbey, R. J., Völker, S. J. Chem. Phys, $1999,110,1010$.

44. Kador, L., Haarer, D., Personov, R. J. Chem. Phys, 1987, 86, 5300.

45. Kador, L., Personov, R., Richter, W., Sesselman, T., Haarer, D. Polymer Journal, 1987, 19,61.

46. Gafert, J., Friedrich, J., Parak, F. Proc. Natl. Acad. Sci. USA, 1995, 92, 2116.

47. Köhler, M., Gafert, J. Friedrich, J., Falk, H., Meyer, J. J. Phys. Chem., 1996, 100, 8567.

48. Milanovich, N., Rätsep, M., Reinot, T., Hayes, J. M., Small, G. J. J. Phys. Chem. B, 1998, 102, 4265 .

49. Milanovich, N., Reinot, T., Hayes, J. M., Small, G. J. Biophys. J., 1998, 74, 2680.

50. Meixner, A. J., Renn, A., Bucher, S. E., Wild, U. P. J. Phys. Chem., 1986, 90, 6777.

51. Altman, R. B., Haarer, D., Renge, I. Chem. Phys. Lett., 1993, 216, 281.

52. Walsh, R. J.; Reinot, T., Hayes, J. M., Kalli, K. R., Hartmann, L. C., Small, G. J. J. Lumin, , 2002, 98, 115.

53. Vauthey, E., Holliday, K., Changjiang, W., Renn, A., Wild, U. P. Chem. Phys., 1993, $171,253$.

54. Köhler, M., Friedrich, J., Fidy, J. Biochim. Biophys. Acta, 1998, 1386, 255. 


\title{
CHAPTER 4. SINGLE-CELL NONPHOTOCHEMICAL HOLE BURNING OF OVARIAN SURFACE EPITHELIAL CARCINOMA AND NORMAL CELLS
}

\author{
A paper published in Proc. Natl. Acad. Sci. U.S.A. 2003, 100(4), 1685. \\ R. J. Walsh, S. Matsuzaki, T. Reinot, J. M. Hayes, \\ K. R. Kalli, L. C. Hartmann, and G. J. Small
}

\begin{abstract}
Persistent spectral nonphotochemical hole burning (NPHB) spectroscopy has recently been applied to dye molecules in cells. The sensitivity of NPHB to the nanoenvironment of the probe is well established. It has been shown that NPHB applied to bulk suspensions of cultured human cells can distinguish between normal and cancer cells. Thus, NPHB has potential as a diagnostic cancer tool. For this reason, the methodology is referred to as hole burning imaging, by analogy with MRI. The optical dephasing time $\left(T_{2}\right)$ of the dye in hole burning image replaces the proton $T_{1}$ relaxation time in MRI. In addition to the $T_{2}$ mode of operation there are four other modes including measurement of the spectral hole growth kinetics (HGK). Reported here is that the selectivity and sensitivity of NPHB operating in the HGK mode allow for distinction between normal and carcinoma cells at the single-cell level. The ovarian cell lines are ovarian surface epithelial cells with temperature-sensitive large $\mathrm{T}$ antigens (analogously normal) and ovarian surface epithelial carcinoma (OV167) cells. The mitochondrial specific dye used was rhodamine 800 (Molecular Probes). This carbocationic dye is highly specific for the outer and inner membranes of mitochondria. In line with the results for bulk suspensions of the two cell lines, the hole burning efficiency for OV167 cells was found to be significantly higher than that for normal cells. Theoretical
\end{abstract}


analysis of the HGK data leads to the conclusion that the degree of structural heterogeneity for the probe-host configurations in OV167 cells is lower than in the normal cells. Possible reasons for this are given.

\section{Introduction}

In seeking to expand on available diagnostic tools for the detection of cancer while improving the level of resolution, this group has previously introduced low temperature intracellular persistent nonphotochemical hole burning (NPHB) [1,2] by using probe dye molecules [3-6]. We refer to this technique as hole burning imaging (HBI), by analogy with MRI (see below). Those works, which involved the study of bulk samples of different cell lines, established that NPHB of the inhomogeneously broadened $\mathrm{S}_{0} \rightarrow \mathrm{S}_{1}$ absorption band of probe molecules in an intraceliular environment is efficient and, moreover, that HBI can be used to distinguish between normal and carcinoma cells. In refs. $[3,4]$ NPHB with the mitochondrial dye rhodamine 800 (MitoFluor Far Red 680 (MF680), Molecular Probes), which is selective for the outer and inner membranes of mitochondria, was used in two modes of operation to distinguish between normal and carcinoma human ovarian surface epithelial cells. We report here that the sensitivity and selectivity of NPHB allow for distinction between single normal and carcinoma ovarian cells.

By way of background, NPHB is a laser-based, site excitation energy-selective spectroscopy that eliminates the large contribution of inhomogeneous broadening (a few

hundred $\mathrm{cm}^{-1}$ ) to the ground to excited-state origin and vibronic absorption bandwidths of a chromophore in complex ("glassy") host matrices [7]. For dye molecules with nanosecond excited-state lifetimes, the improvement in resolution at $\sim 4 \mathrm{~K}$ can be as high as $10^{4}$, as 
determined by the ratio of the inhomogeneous broadening of the origin absorption band to the width of a laser-limited hole burned into that band. Such a hole is referred to as a zerophonon hole (ZPH) and corresponds to the zero-phonon line in absorption [7]. The zerophonon line in a solid is analogous to a pure electronic transition of a gas-phase molecule to which rotations and molecular vibrations do not contribute. For the above rhodamine dye (hereafter MF680) in ovarian cells, the $\mathrm{ZPH}$ widths at $2 \mathrm{~K}$ are $\sim 0.1 \mathrm{~cm}^{-1}$, whereas the inhomogeneous broadening is $\sim 800 \mathrm{~cm}^{-1}[3,4]$. (It follows from theory that the width of the $\mathrm{ZPH}$ is twice the width of the zero-phonon line.) Glass-like structural disorder of the host, such as exists in glasses, polymers and proteins, is an essential ingredient for NPHB. Production of a spectral hole is a consequence of tunneling between nearly isoenergetic bistable host-chromophore configurations that is triggered by electronic excitation of the chromophore. The bistable configurations are often referred to as two-level systems (TLS). On completion of the ground-state to excited-state to ground-state cycle, the chromophore is trapped in a nanoenvironment that is different from that before excitation. As a result, the absorption frequency of the chromophore is shifted to another position within the inhomogeneously broadened absorption band, thus the production of a ZPH at the laser burn wavelength $\lambda_{\mathrm{B}}$. The ZPH can persist for months, provided the sample is kept at or below the burn temperature and in the dark to avoid light-induced hole filling. The resulting positive absorption at other wavelengths, referred to as the antihole, usually has a width on the order of the inhomogeneous width. The reader is referred to Reinot and Small [8] for a detailed discussion of the NPHB mechanism. Briefly, the TLS can be subdivided into those intrinsic to the glassy host $\left(\mathrm{TLS}_{\mathrm{int}}\right)$ and those associated with the dye molecule and its inner shell of solvent molecules, extrinsic TLS (TLS $\mathrm{ext}_{\mathrm{t}}$ ). Probe excitation results first in tunneling of 
TLS int in the outer shell, which are intimately connected with the excess free volume of glasses [9]. This tunneling leads to diffusion of excess free volume to the inner shell, opening the way for the rate-determining step of NPHB, which is phonon-assisted tunneling of the $\operatorname{TLS}_{\text {ext }}$. The tunneling rate is written as $\mathrm{R}=\Omega \exp (-2 \lambda)$, where $\lambda$ is the tunnel parameter, and $\Omega$ is proportional to the square of the tunneling frequency of the TLS $S_{\mathrm{ext}}[10]$. NPHB has been widely used for investigating the spectral dynamics of chromphores in glasses and polymers [11-18], due to tunneling of $T L S_{\text {int }}$ and for the excited-state electronic structure and excitation energy transfer/electron transfer processes of photosynthetic proteinchlorophyll complexes [19-24]. NPHB is very sensitive to the nanoenvironment of the probe chromophore and thus its use in differentiation between normal and cancer cells is a logical application.

Four properties of the probe molecules that can be determined by using the ZPH. (i) Its optical dephasing time $\left(T_{2}\right)$ that is temperature dependent and determined by the homogeneous width of the ZPH [25]: $T_{2}$ replaces the $T_{1}$ proton relaxation time in MRI. (ii) The dispersive (nonsingle exponential) hole growth kinetics (HGK) that reflect structural heterogeneity [10]. (iii) The permanent dipole moment change $(\Delta \mu)$ of the $S_{0} \rightarrow S_{1}$ transition that is obtained by measuring the response of the ZPH (broadening and/or splitting) to an external electric (Stark) field [26]. Here it is the matrix-induced contribution to $\Delta \mu$ that is of interest. (iv) The local compressibility ( $\kappa$ ) that is determined from the linear pressure shift rate of the ZPH frequency [27]. In addition, one can monitor the phonon (low-frequency intermolecular mode) sideband hole structure associated with the $\mathrm{ZPH}$. It is the strength of the transition electron-phonon coupling that determines the relative intensities of the ZPH and phonon sideband hole structure. 
The studies of Walsh and colleagues $[3,4]$ with MF680 used two human epithelial ovarian cell lines: one, a carcinoma cell line (ovarian surface epithelial carcinoma cells (OV167)) derived from a high-stage high-grade serous tumor, the most commonly diagnosed form of ovarian cancer; and the second, a line derived from normal ovarian surface epithelium [28] (see below). Specificity of MF680 for mitochondria was demonstrated by mean of confocal microscopy and spectroscopy. (MF680 is a carbocationic lipophilic dye that locates preferentially in mitochondrial membranes due to their lipid-enriched composition and lowered membrane potential relative to extra-mitochondrial areas.) Stringent cryopreservation protocol was used. Of the five aforementioned NPHB properties. it was found that NPHB operating in the HGK mode and Stark $(\Delta \mu)$ mode provided the highest degree of differentiation between the two cell lines. The $\Delta \mu$ value for the OV167 cells was found to be a factor of 1.5 larger than that of the normal cells. It was suggested that the difference may result from the membrane potential $\left(\Delta \Psi_{m}\right)$ of the carcinoma cells being larger than that of the normal cells [3]. The HGK data revealed that the OV167 cells burn significantly more efficiently than the normal cells. Theoretical analysis of the data revealed that the degree of structural disorder for the OV167 cells is the lower of the two, as is the average value of the tunnel parameter $\left(\lambda_{0}\right)$. It was emphasized that HBI operating in the HGK mode has the advantage that only inexpensive diode lasers are required rather than tunable lasers required for the other modes of operation [4].

In this paper, HGK differences are demonistrated for single cells of the model cell lines, with trends similar to our previous observations for bulk suspensions containing $0.5-2.0 \times 10^{6}$ cells, thus demonstrating the potential of HGK diagnostics with resolution at 
the single-cell level. As in refs.[3,4], the HGK curves were obtained by monitoring the decrease in fluorescence that accompanies burning of spectral holes.

\section{Materials and Methods}

Cell Culture. Pertinent information regarding the choice of model cell lines was covered in a recent article [4]. Only relevant growth culture information is given here.

Ovarian surface epithelial carcinoma (OV167) cells were grown in Alpha MEM Earle's salts with nucleosides (Irvine Scientific), supplemented with 20\% FBS, penicillin/streptomycin (100 units/ml and $100 \mu \mathrm{g} / \mathrm{ml}$, respectively) and L-Glutamine ( $2 \mathrm{mM}$ final concentration, resupplemented every 2 wk). For each hole burning experiment, cells were cultured for 6-7 days (medium resupplementation on day 3) in a $25 \mathrm{~cm}^{2}$ culture flask and then transferred to an $18 \mathrm{~mm}$ square glass poly-L-lysine treated coverslip for 24 hours before staining and cryofixation. Cells were incubated at $37^{\circ} \mathrm{C}$ and $5.5 \% \mathrm{CO}_{2}$ atmosphere.

For analogously normal cells, short-term normal ovarian surface epithelial (OSE) cell

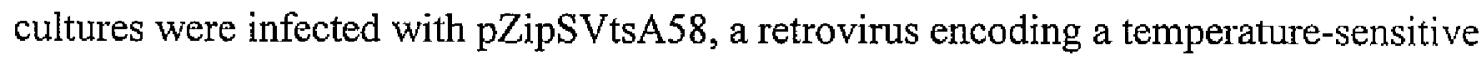
mutant of the simian virus 40 large $\mathrm{T}$ antigen; for simplicity, we will refer to them as OSE(tsT)-14. OSE(tsT)-14 cells were cultured in a 1:1 mixture of Medium 199 and MCDB105 medium mix, supplemented with 15\% FBS, penicillin/streptomycin (100 units/m] and $100 \mu \mathrm{g} / \mathrm{ml}$, respectively) and L-Glutamine( $2 \mathrm{mM}$ final concentration, resupplemented every $2 \mathrm{wk}$ ). Cells were expanded at the permissive temperature of $34^{\circ} \mathrm{C}, 5.5 \% \mathrm{CO}_{2}$ for $6-7$ days (medium resupplementation on day 3), transferred to a glass poly-L-lysine treated coverslip 24 hours before use, and cultured at $39^{\circ} \mathrm{C}, 5.5 \% \mathrm{CO}_{2}$ for $12-18$ hours (allowing degradation of the heat-sensitive large $\mathrm{T}$ antigen) before staining and cryofixation. 
Cells were stained using MF680 (Molecular Probes) for 15 minutes at a concentration of $100 \mathrm{nM}$. MF680 was initially dissolved in DMSO. At concentrations used for staining (after diluting with PBS and subsequently growth medium), the DMSO component of the staining medium was negligible (0.0002\%). OV167 and OSE(tsT)-14 cells were removed from the culture flask using $2 \times$ and $1 \times$ trypsin/EDTA $(2.5 \mathrm{~g} / \mathrm{L} / 1 \mathrm{mM}$ stock) solutions, respectively. For multiple runs, cells were used at the same passage number. Unless otherwise noted, all chemicals were purchased from Sigma.

Laser System. A schematic of the fluorescence data collection system is shown in Fig. 1. In brief, a ring dye laser using LD 688 dye (Exciton, Dayton, $\mathrm{OH}$ ) was pumped by a Coherent Innova 90-6 argon ion laser (Coherent, Santa Clara, CA). The laser system provided $100-500 \mathrm{~mW}$ of power over a wavelength region of $660-720 \mathrm{~nm}$. Laser intensity was stabilized at $50 \mathrm{~mW}$ by an LS100 laser power stabilizer (Cambridge Research and Instrumentation, Cambridge, MA), and the laser beam was focused into a multimode fiber optic with a $5 \times$ microscope objective. At the output end of the fiber optic, polyethylene sheets were utilized to reduce the spot power to $\sim 1 \mathrm{~mW}$ and to diffuse the laser light for uniform illumination of the entire area imaged. Laser intensity during burns was $\sim 250 \mu \mathrm{W} / \mathrm{cm}^{2}$. Two low pass filters, one a $720 \mathrm{~nm}$ cutoff filter (model no. 720ALP, Omega Optical, Brattleboro, VT) and the other a $750 \mathrm{~nm}$ cutoff filter placed between the cryostat window and charge-coupled device (CCD) served to remove excitation wavelengths. For burns, the laser was operated with intracavity etalons (linewidths $<0.0003 \mathrm{~cm}^{-1}(<10 \mathrm{MHz})$ ) installed. Laser fluence for hole burning was varied with the use of a series of neutral density (gray) filters. Excitation wavelengths are noted in figures. 
Cryostat / Fluorescence Signal Collection. Coverslips with adherent cells of both types in separate wells were mounted on a sample manipulator (Fig. 1, inset) and plunged into a Janis cryostat (model STVP-100, Janis Research, Wilmington, MA) precooled to near

liquid helium temperature $(\sim 5 \mathrm{~K})$ [29]. The sample manipulator allowed for movement in $\mathrm{x}$, $y$, and $z$ directions. $A \times 25$-reflecting objective was mounted in the cryostat, with the image redirected out of the cryostat via a prism. The fluorescence image was collected using a CCD camera (model LN/CCD-1340/400-EB/1, Roper Scientifc, Trenton, NJ) controlled by WinView32 software (version 2.4.1.15, Roper Scientific). Images were collected at equivalent intervals, with identically sized regions of interest. Typically, 900 images were collected during a burn.

\section{Results and Discussion}

Fig. 2 shows a series of ZPH burned at 1-nm intervals with a constant burn fluence of $10 \mathrm{~mJ} / \mathrm{cm}^{2}$ for a bulk suspension of MF680 in OV167 cells. This ZPH "action" spectrum was obtained in the fluorescence excitation mode and is the difference between the post- and preburn absorption spectra. For burn wavelengths $\left(\lambda_{B}\right) \geq 704 \mathrm{~nm}$, the fractional ZPH depths were found to be constant within experimental uncertainty. (The intensity fluctuations in Fig. 2 for $\lambda_{B} \geq 704 \mathrm{~nm}$ are due to the step size of the laser scan being about five times larger than the width of the ZPH.) The action spectrum of OV167 in the OSE(tsT)-14 cells is similar [29]. Thus, for both cell lines, HGK curves can be measured at a large number of wavelengths by monitoring the decrease in fluorescence intensity as hole burning proceeds. The decrease in hole intensities at wavelengths shorter than $\sim 704 \mathrm{~nm}$ is due to the absorption being increasingly dominated by vibronic/phononic transitions rather than the pure electronic 


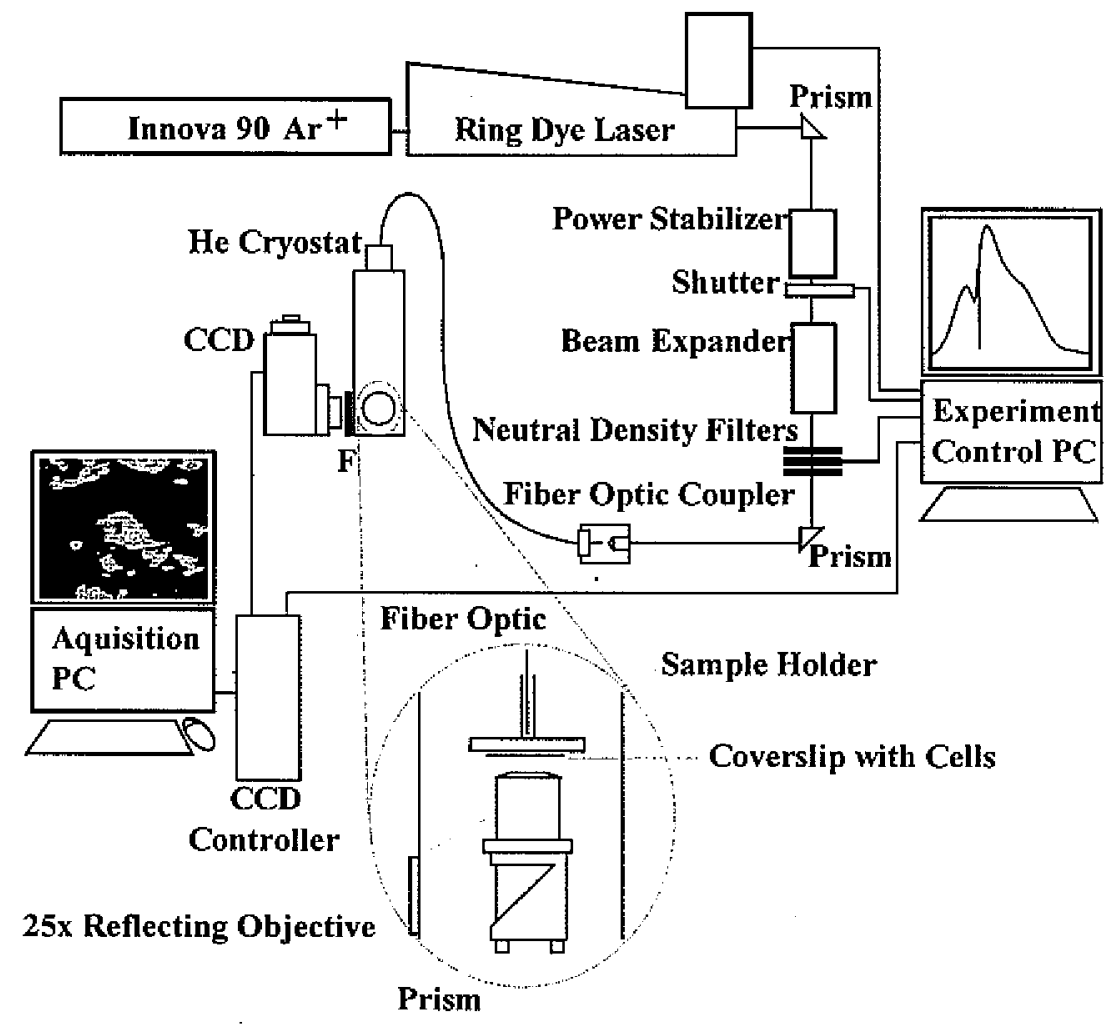

Figure 1 Schematic of laboratory setup for imaging cells by laser-induced fluorescence and subsequent calculation of HGK. "F" refers to a low pass filter to remove excitation frequencies. Inset shows detail of the sample holder/manipulator and reflecting objective/prism configuration. 


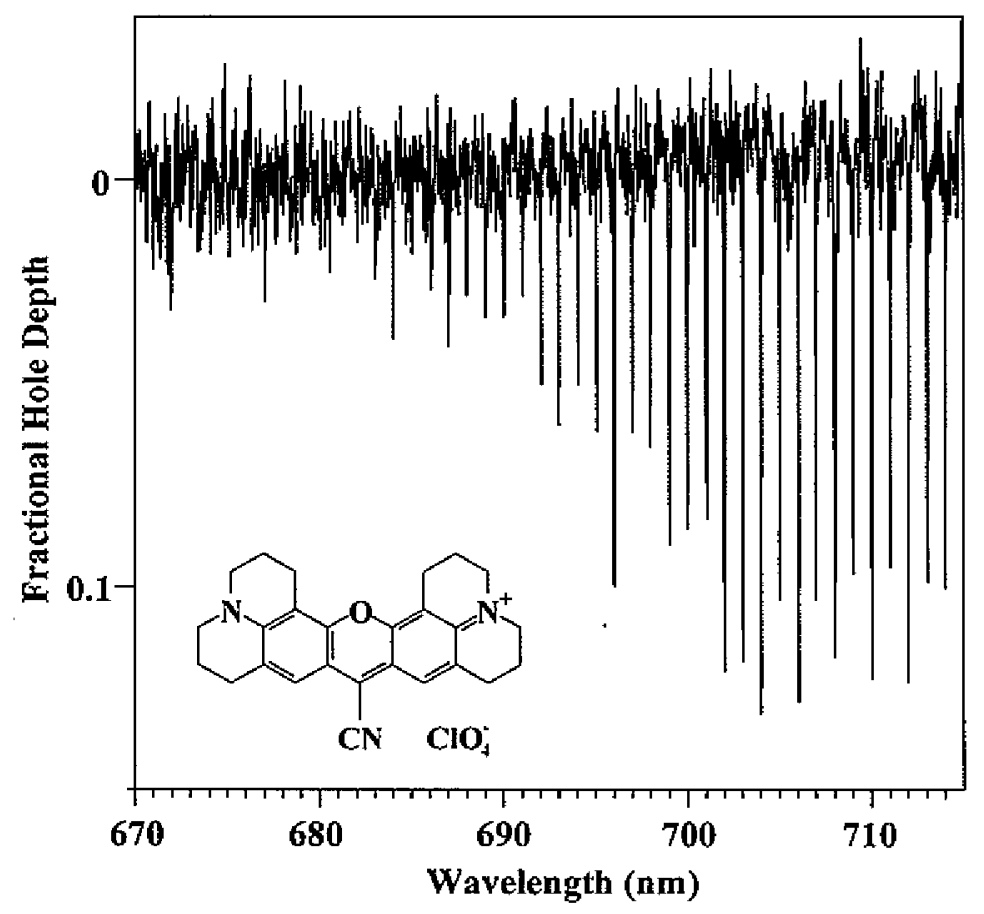

Figure 2 A set of zero-phonon holes (the action spectrum) of MF680 in OSE(tsT)-14 normal cells. Each hole was burned at $\mathrm{T}=2.0 \mathrm{~K}$ with a fluence of $10 \mathrm{~mJ} / \mathrm{cm}^{2}$ at 1-nm intervals; burning started at $675 \mathrm{~nm}$ proceeding towards $715 \mathrm{~nm}$. The structure of the dye molecule, MF680, is shown in (inset). 
transition. The burn fluence of $10 \mathrm{~mJ} / \mathrm{cm}^{2}$ is too low to reveal the phonon sideband hole structure that accompanies the ZPH. Such structure evolves at higher burn fluences [4]. A representative image of OV167 cells stained with MF680 is presented in Fig. 3. HGK curves were generated by collecting sequential fluorescence images, followed by analysis of specific regions of interest for signal intensity, as depicted by the white boxsuperimposed on one of the cells in Fig. 3A. For each frame of a series of images, each pixel contained within the box was summed and plotted against frame number, resulting in a HGK "image" as shown in Fig. 3C. By this method, numerous cells were analyzed individually for comparison. For simplicity, only frame numbers are quoted, regardless of frame exposure time used (which correspond to $1 \mathrm{~s}$ in the present case). However, exposure time does not include the time taken to read and report the CCD chip, which is a significant time contribution. For a frame with 1 -s exposure, $0.1-0.4 \mathrm{~s}$ of additional time is added by the read and report process, dependent on the size of the image frame (regions of the CCD chip can be ignored during data collection, thus the region of interest must be set the same for accurate comparisons). The data presented in this case has not been corrected for this additional time. Because all data presented here were collected under identical conditions by using identical frame sizes, this is not seen as a significant problem.

Structural heterogeneity of amorphous solids leads to a distribution of values for the tunnel parameter $\lambda$ of the extrinsic TLS. A Gaussian for the distribution function $f(\lambda)$ has been shown to be physically reasonable (ref. [10] and references therein). The distribution center and standard deviation are denoted by $\lambda_{0}$ and $\sigma_{\lambda}$. For each cell line, the HGK curves were fit [8] by using 
Relative Intensity $\longrightarrow$

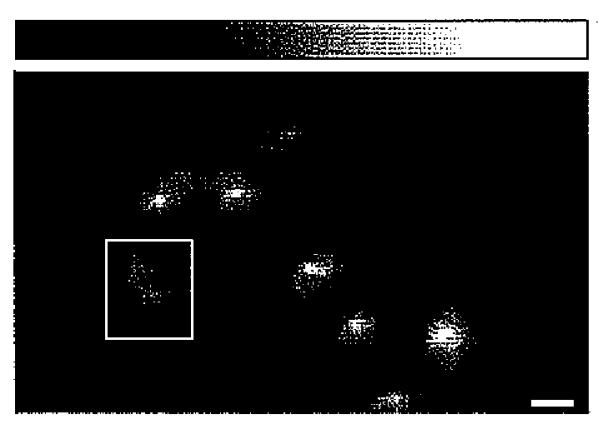

$\mathbf{A}$
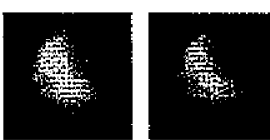

1
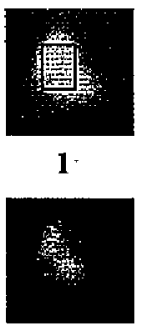

200

$\mathbf{B}$

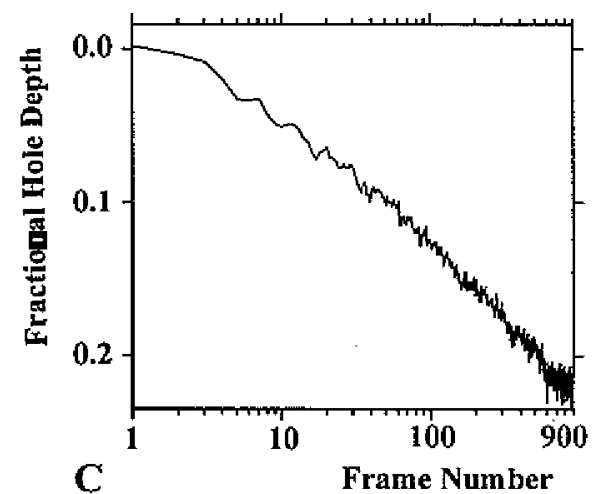

Figure 3 Initial image (A) of OV167 cells stained with MF680 and burned at $\lambda_{B}=710$ $\mathrm{nm}$. The white bar represents $20 \mu \mathrm{m}$. In $\mathrm{B}$, individual images of the same cell at increasing frame number are shown, exhibiting the burn over time. The cell shown corresponds to the white box shown in $\mathrm{A}$. In $\mathrm{C}$, a hole growth kinetics curve has been created from the individual cell shown in $\mathrm{B}$ by summing the pixels contained within the box superimposed over the cell image at frame 1 and repeated for each of the 900 image frames. Other conditions: $T=6.8 \mathrm{~K}$ : time for each exposure: $1 \mathrm{~s}$; laser intensity $(P)=250 \mu \mathrm{W} / \mathrm{cm}^{2}$. 


$$
D(t)=\int d \lambda f(\lambda) \int d \alpha \sin \alpha \cos ^{2} \alpha\left(e^{-P \alpha L_{T} \phi(\lambda) \cos ^{2} \alpha d}\right)
$$

where $1-D(t)$ is the fractional hole depth after a burn for time $t$ at a burn frequency $\omega_{\mathrm{B}} . P$ is the photon flux (number of photons $\mathrm{cm}^{-2} \mathrm{~s}^{-1}$ ), $\alpha$ is the angle between the laser polarization and transition dipole of the chromophore, and $\sigma_{\mathrm{LT}}^{\mathrm{P}}$ is the peak absorption cross section $(2.8 \times$ $10^{-12} \mathrm{~cm}^{2}$ for MF680 [4]). The NPHB quantum yield is given by

$$
\phi(\lambda)=\frac{\Omega \exp (-2 \lambda)}{\Omega \exp (-2 \lambda)+\tau^{-1}}
$$

where $\Omega \exp (-2 \lambda)$ represents the phonon-assisted tunneling rate; $\Omega$ is set to $7.6 \times 10^{12} \mathrm{~s}^{-1}$ [10]; and $\tau$, the fluorescence lifetime, was determined to be $1.8 \mathrm{~ns}$ at $77 \mathrm{~K}$. The parameters $\lambda$ and $\alpha$ each cause a distribution of hole burning rates that leads to dispersive kinetics, and it has been shown that the $\lambda$ distribution is by far the dominant factor for the first $80 \%$ of the total burn [8]. $\sigma_{\lambda}$ values for the $\lambda_{0}$ parameter allow conclusions to be drawn regarding the relative order of the environment experienced by the chromophore, which in the present case consists of MF680 in the lipid-based mitochondrial membranes.

Representative curves fit by Eq. 1 are presented in Fig. 4, where the fits are superimposed over data curves. The value of $P$ is given in the caption. $\lambda_{0}$ and $\sigma_{\lambda}$ were adjustable parameters. When fitting the kinetics data it is necessary to know the Huang-Rhys factor $(S)$, which defines the strength of the electron-phonon coupling, because the maximum depth of the $\mathrm{ZPH}$ is $\exp (-S)$ in the low-temperature limit. To obtain good fits that were also physically reasonable, an $S$ value of $\sim 1.4$ was required which is larger than the value of 1.1 determined in ref. [4] for both cell lines. In Fig. 4A, three HGK curves are presented for cells of each type of cell line, with the observation that the OV167 carcinoma cell line burns 

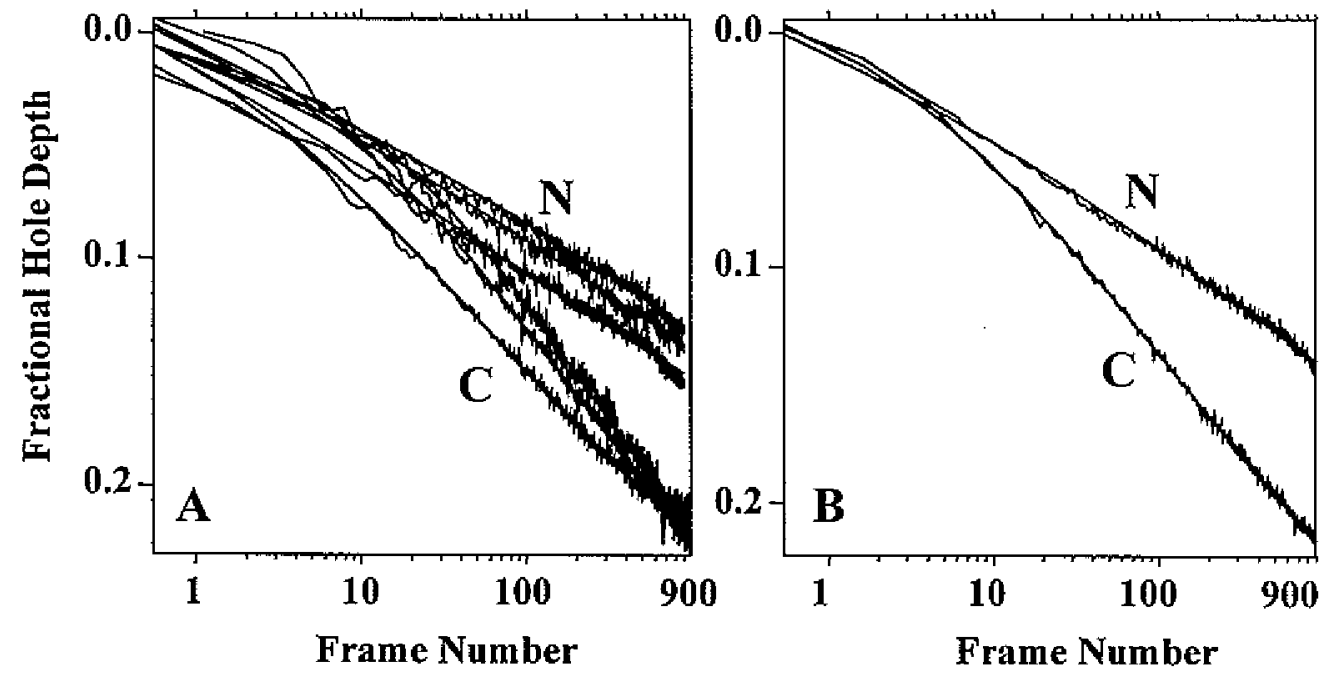

Figure 4 A. Representative HGK data and fit curves generated for three of each cell line from subsequent images of OV167 carcinoma cells (C) and OSE(tsT)-14 normal analogs $(N)$. In $\mathbf{B}$, HGK curves from 15 different cells were summed and fit. $P=250 \mu \mathrm{W} / \mathrm{cm}^{2} ; \mathrm{T}=6.8 \mathrm{~K}$. 
to a deeper fractional hole depth in the same number of frames (equivalent to the same amount of burn time) relative to the OSE(tsT)-14 normal analogs. This trend is in agreement with our previous observations on cells grown and analyzed in bulk. Noteworthy from Fig. 4A, not all of the cells in the same cell line burn at identical rates. Rather, cells tend to show distinct rates by comparison, indicative of heterogeneities between cells in the same line but in keeping with the overall trends observed between the two cell lines.

Complete initial images of each cell line are presented in Fig. 5. Superimposed on individual cells are numbers corresponding to the fit parameters listed to the side of the image. From the fit parameters, heterogeneities in burn rates are evident, and further analysis of the fit parameters gives values for the OV167 carcinoma line of $\bar{\lambda}_{0}$ and $\bar{\sigma}_{2}$ and values for the OSE(tsT)-14 normal line of $\bar{\lambda}_{0}$ and $\bar{\sigma}_{\lambda}$. (The error limits are standard deviations of all of the measurements.) The values of $\bar{\lambda}_{0}$ are statistically indistinguishable, indicating that the cellular environments of the dye in the two cell types are similar. On the other hand, the mean values of $\sigma_{\lambda}$ for the two cell types are significantly different. At the $99 \%$ confidence level, the value for the OV167 cells would lie between 1.1 and 1.4, whereas the range for the OSE(tsT)-14 cells is from 1.7 to 2.0 . However, for a single cell, there is about a $5 \%$ probability that the cell would be misidentified only on the basis of a measurement of $\sigma_{\lambda}$. One further point about the above averages is that the values of $\lambda_{0}$ and $\sigma_{2}$ are uncorrelated (correlation coefficients $=0.2$ for OV167 and 0.5 for OSE(tsT)-14, respectively). In comparison to the single-cell averages, previously reported HGK fit parameters for bulk cell suspensions gave $\lambda_{0}$ and $\sigma_{\lambda}$ for OV167 cells and $\lambda_{0}$ and $\sigma_{\lambda}$ for the OSE(tsT)-14 cell line [4]. The trend for lower $\sigma_{\lambda}$ values in the carcinoma line is identical, but the values differ. This 


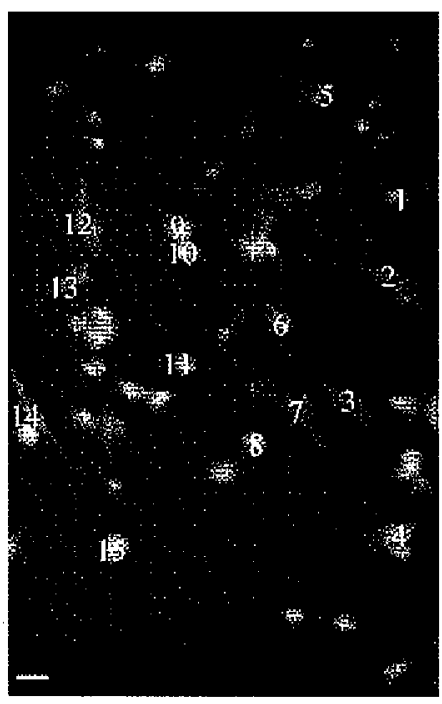

OSE(tsT) 14 Dispersive

HGK Fit Parameters

$\begin{array}{ccc}\text { Cell } & \lambda_{\mathrm{o}} & \sigma_{i} \\ 1 & 8.5 & 1.88 \\ 2 & 8.6 & 2.01 \\ 3 & 8.6 & 1.84 \\ 4 & 9.0 & 1.81 \\ 5 & 8.7 & 1.81 \\ 6 & 8.3 & 1.54 \\ 7 & 8.7 & 2.02 \\ 8 & 8.7 & 2.14 \\ 9 & 8.4 & 1.65 \\ 10 & 8.2 & 1.91 \\ 11 & 8.4 & 1.83 \\ 12 & 8.5 & 2.09 \\ 13 & 8.1 & 1.77 \\ 14 & 7.5 & 1.64 \\ 15 & 8.5 & 2.01\end{array}$

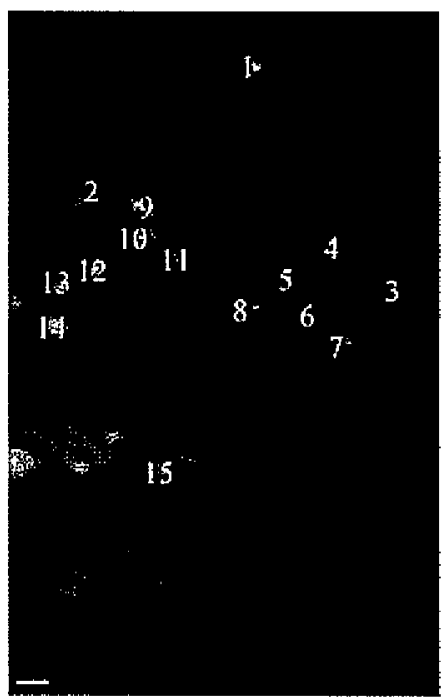

OV167 Dispersive HGK Fit Parameters

\begin{tabular}{rcc}
\hline Cell & $\lambda_{\mathrm{a}}$ & $\sigma_{i}$ \\
\hline 1 & 8.7 & 1.38 \\
2 & 8.8 & 1.07 \\
3 & 8.2 & 1.11 \\
4 & 8.7 & 1.32 \\
5 & 8.5 & 1.27 \\
6 & 8.4 & 1.12 \\
7 & 8.6 & 1.20 \\
8 & 8.3 & 1.43 \\
9 & 8.9 & 1.45 \\
10 & 8.8 & 1.20 \\
11 & 8.3 & 1.16 \\
12 & 8.5 & 1.49 \\
13 & 8.5 & 1.29 \\
14 & 8.6 & 1.27 \\
15 & 8.5 & 1.00
\end{tabular}

Figure 5 Images of OV167 carcinoma and OSE(tsT)-14 normal cells and concurrent HGK fit parameters. 
difference is likely contributed to by differences in experimental setups. The present method does not afford the signal sampling sensitivity that is capable with detection by a photomultiplier tube as in ref. [4]. In that study HGK data points were collected every $0.1 \mathrm{~s}$ for the first $30 \mathrm{~s}$, providing high resolution of the first $30-50 \%$ of the entire burn depth. After $30 \mathrm{~s}$, data points were collected less frequently $\left(1 \mathrm{~s}^{-1}\right)$. In the present case, however, images were collected every $\sim 1 \mathrm{~s}$ for the entire burn, limiting the resolution of the initial part of the burn, which is of primary importance in arriving at reliable values for $\sigma_{\lambda}$. As a result, a straight comparison of HGK fit parameters between bulk suspensions ard single cells is not warranted. Another difference between the bulk and single cell studies is that in the former, the samples were frozen under conditions that optimize cryopreservation, whereas in the latter, coverslips with adherent cells were frozen to optimize cryofixation [30]. It is possible that the faster cooling rate with cryofixation traps a wider distribution of chromophore-host configurations, which translates into larger $\sigma_{\lambda}$ values. Larger $\sigma_{\lambda}$ values might also arise from straining of cells due to their adherence to the coverslips.

A second HGK comparison was also conducted by summing all of the kinetics curves for individual cells (Fig. 4B) and fitting the resulting curve, with the result of $\sigma_{\lambda}=2.0$ and $\lambda_{0}$ $=8.5$ for the OSE(tsT)-14 cells and $\lambda_{0}=1.2$ and $\lambda_{0}=8.5$ for the OV167 cells. These values are within the experimental uncertainty of the individual cell evaluations, exhibiting good agreement between the two methods used.

One familiar with Eq. I would immediately conclude from the experimental HGK curves in Fig. 4 (as well as those for bulk cell suspensions given in refs. $[3,4]$ ) that the standard deviation $\sigma_{\lambda}$ is smaller for OV167 cells, which indicates that heterogeneity of MF680 sites in the membranes of those cells is less severe than in the analogously normal 
OSE(tsT)-14 cells. At this time, we can only speculate on the reason(s) for this finding. One possibility emerges from the confocal fluorescence images presented in ref. [4]. They showed that the OV167 cell line exhibits relatively small, more cobblestone-like shapes for the majority of the cells, and that the cells form highly aggregated structures relative to the OSE(tsT)-14 cells. This is presumably due, in part, to the very rapid proliferation rate of the carcinoma line. Long networks of mitochondria were not formed within the time frame used in those experiments. Such networks were observed in the normal cells, because with passage, these cells changed from the cobblestone morphology to a fibroblast-like morphology as previously characterized [31]. The regular and aggregate cobblestone structure of the carcinoma line would seem to correlate with a decrease in structural heterogeneity. Another possibility is that the higher membrane potential of cancer cells relative to normal cells (by a factor of $\sim 1.5[3,4]$ ) might lead to greater structural order due to enhancement of electrostatic interactions.

In conclusion, we have shown, to our knowledge for the first time, that persistent NPHB of a chromophore in single cells is feasible. In the HGK mode, HBI with the membrane-selective mitochondrial dye MF680 proved capable of distinguishing between single cancer and normal ovarian epithelial cells. However, a shortcoming of MF680 is that its hole burning efficiency is too high. This prevented us from using the other operational modes of HBI that require scanning of the burn laser to obtain hole-burned spectra. Efforts are underway to find less efficient dyes that are selective for specific organelles. We have recently shown that $\mathrm{HBI}$ can be applied to cancerous tissue (unpublished results). It will be interesting to see whether HBI can distinguish between normal and cancerous tissue. 


\section{Acknowledgement}

T. R. was supported by the Solid State Chemistry and Polymers Program of the National Science Foundation (Grant DMR-9908714). Ames Laboratory is operated for the

U. S. Department of Energy by Iowa State University under Contract W-7405-Eng-82. This work was supported by the Office of Biological and Environmental Research.

\section{References}

1. Personov, R. I., Al'shitz, E. I., Bykovskaya, L. A.JETP Lett., 1972, 15, 609.

2. Hayes, J. M., Small, G. J. Chem. Phys., 1978, 27, 151.

3. Walsh, R. J., Reinot, T., Hayes, J. M., Kalli, K. R., Hartmann, L. C., Small, G. J. J. Luminesc., 2002, 98, 115.

4. Walsh, R. J., Reinot, T., Hayes, J. M., Kalli, K. R., Hartmann, L. C., Small, G. J. Biophys. J., 2003, 84, 1299.

5. Milanovich, N., Reinot, T., Hayes, J. M., Small, G. J. Biophys. J., 1998, 74, 2680.

6. Milanovich, N., Rätsep, M.; Reinot, T., Hayes, J. M., \& Small, G. J. J. Phys. Chem. $B, \mathbf{1 9 9 8}, 102,4265$.

7. Rebane, K. K. in Zero-Phonon Lines and Spectral Hole Burning in Spectroscopy and Photochemistry (Sild, O., Haller, K. eds.), 1988, Springer, Heidelberg, pp. 1-19.

8. Reinot, T., Small, G. J. J. Chem. Phys., 2001, 114, 9105.

9. Cohen, M., Grest, G. S. Phys. Rev. B, 1979, 20, 1077.

10. Kenney, M. J., Jankowiak, R., Small, G. J. Chem. Phys, 1990, 146, 47.

11. Van den Berg, R., Völker, S. Chem. Phys., 1988, 128, 257.

12. Reinot, T., Hayes, J. M., Small, G. J. J. Chem. Phys., 1997, 106, 457.

13. Reinot, T., Kim, W. H., Hayes, J. M., Small, G. J. J. Opt. Soc. Am. B, 1997, 14, 602.

14. Reinot, T., Hayes, J. M., Small, G. J. J. Chem. Phys., 1999, $110,4820$. 
15. Silbey, R. J., Koedijk, J. M. A., Völker, S. J. Chem. Phys., 1996, 105, 901.

16. Walsh, C. A., Berg, M., Narasimhan, L. R., Fayer, M. D. J. Chem. Phys., 1987, 86, 77.

17. Hayes, J. M., Reinot, T., Small, G. J. Chem. Phys. Lett., 1999, 312, 362.

18. Kador, L., Personov, R., Richter, W., Sesselmann, T., Haarer D. Polymer J., 1987, 19. 61.

19. Reddy, N. R. S., Kolaczkowski, S. V., Small, G. J. Science, 1993, 260, 68.

20. Rätsep, M., Johnson, T. W., Chitnis, P. R., Small, G. J. J. Phys. Chem. B, 2000, 104, 836.

21. Rätsep, M., Wu, H.-M., Hayes, J. M., Blankenship, R. E., Cogdell, R. J., Small, G. J. $J$. Phys. Chem. B, 1998, 102, 4035.

22. Rätsep, M., Wu, H.-M., Hayes, J. M., Small, G. J. Spectrochim. Acta Part A, 1998, 54A, 1279.

23. Wu, H.-M., Rätsep, M., Young, C. S., Jankowiak, R., Blankenship, R. E., Small, G. J. Biophys. J., 2000, 79, 1561.

24. Zazubovich, V., Matsuzaki, S., Johnson, T. W., Hayes, J. M., Chitnis, P. R., Small, G. J. Chem. Phys., 2002, 275, 47.

25. Völker, S. in Relaxation Processes in Molecular Excited States (Fünfschilling, J, ed.), 1989, Kluwer, Dordrecht, pp. 113.

26. Köhler, M., Friedrich, J., Fidy, J. Biochim. Biophys. Acta, 1998, 1386, 255.

27. Sesselmann, T., Richter, W., Haarer, D. J: Luminesc., 1987, 36, 263.

28. Conover, C. A., Hartmann, L. C., Bradley, S., Stalboerger, P., Klee, G. G., Kalli, K. R., Jenkins, R. B. Exp. Cell Res., 1998, 238, 439.

29. Walsh, R. J. Ph.D. Dissertation, 2002, Towa State University.

30. Wisniewski, R. BioPharm., 1998, 11, 42.

31. Auersperg, N., Maines-Bandiera, S. L., Dyck, H. G., Kruk, P. A. Lab. Invest., 1994, 71,510 . 


\title{
- CHAPTER 5. NONPHOTOCHEMICAL HOLE BURNING STUDY OF A MITOCHONDRIAL SELECTIVE RHODAMINE DYE IN CANCEROUS AND NORMAL HUMAN OVARIAN TISSUES
}

\author{
A paper to be submitted to $J$. Phys. Chem. B
}

S. Matsuzaki, J. M. Hayes, G. J. Small, D. M. Benbrook, and R. Jankowiak

\begin{abstract}
By examining the results of nonphotochemical hole burning experiments of cancerous ovarian and analogous normal peritoneal in vitro tissues stained with rhodamine 800 , we find that to date, rhodamine 800 is in fact the most suitable known dye to be used when implementing the hole burning technique as a cancer diagnostic tool. Although in comparing the fluorescence excitation spectra, dispersive hole growth kinetics, and external electric field (Stark) effects observed from each sample while using this dye, only the Stark effects showed a significant difference, this difference was significant enough to solicit direct attention. For the cancerous tissue, the permanent dipole moment change for the $S_{0} \rightarrow S_{1}$ transition of the dye was measured at a factor of 1.35 higher than that of the normal tissue. With comparing the result to the earlier hole burning studies of human ovarian surface epithelial cell lines: OV167 carcinoma cells and OSE(tsT)-14 normal cells (Walsh et al., Biophys. J., 2003, 84, 1299), it is speculated that the difference in the permanent dipole moment change may be related to differences in mitochondrial membrane potentials in these tissue samples.
\end{abstract}




\section{Introduction}

Nonphotochemical hole burning (NPHB) of several dye molecules in various polymers and glasses has been extensively studied to show that NPHB is sensitive to the microenvironment around the dye [1-7]. In recent papers [8,9], hole burning imaging ( $\mathrm{HBI}$ ), a technique that seeks to exploit the inherent nano-environmental sensitivity of NPHB for detecting quantifiable differences between cancerous and analogous normal cells, has been explored. The objective of this work was to establish the procedural feasibility of HBI as a diagnostic tool and to demonstrate NPHB of intra-cellular dye molecules can be used to "image" anomalies in subcellular structures. Specifically, the above studies used two distinct cultured human ovarian surface epithelial cell lines: OV167 carcinoma and OSE(tsT)-14 temperature-sensitive SV40 transfected normal cells, both stained with the mitochondrion specific dye, Molecular Probes MitoFluor Far Red 680 (MF680), commonly known as rhodamine 800 .

In earlier work by this group $[10,11]$, an analogy was drawn between $\mathrm{HBI}$ and magnetic resonance imaging (MRI). This analogy was based on the fact that pure dephasing times $\left(T_{2}^{*}\right)$ are measured in HBI instead of proton relaxation times $\left(T_{1}\right)$, with common relation existing between the two in total dephasing time $\left(T_{2}\right)$. It should be noted that the measurement of $T_{2}$ might be useful in detecting cellular/tissue anomalies which has been suggested by the work of Furusawa et al. [12] regarding the variations of photon echo decay of animal and human tissue samples stained with various thodamine dyes. However, the earlier hole width measurements for cells stained with a phthalocyanine dye, aluminum phthalocyanine tetrasulfonate (APT), could not successfully differentiate between a carcinoma cell line and a related nondiseased cell line, where the hole width is the hole 
burning parameter most directly dependent on $T_{2}$. With HBI, however, a variety of other parameters can be measured, namely the electron-phonon coupling, the response of the hole width to external electric (Stark) fields, and pressure [11].

In Walsh et al. [8,9], although no differences between the above-mentioned two cell lines were detected for zero-phonon hole (ZPH) widths or temperature-dependent ZPH widths, a significant difference in the dipole moment change was measured by the electric field effect (Stark effect). The width of the ZPH depends on an applied electric field ( $\mathbf{E}_{\mathrm{ST}}$ ) with laser excitation $\left(\mathbf{E}_{\mathrm{L}}\right)$ either parallel or perpendicular to $\mathbf{E}_{\mathrm{\$ T}}$. This dependence yielded the permanent dipole moment change associated with the $S_{0} \rightarrow S_{1}$ optical transition of MF680. The MF680 molecules sensitive to the Stark effect were argued to be preferentially located on the mitochondrial membrane. The difference between the dipole moment change values for the two cell lines was up to $\sim 0.8 \mathrm{D}$ and the dipole moment change values for the carcinoma cell line was a factor of 1.5 higher at various burning frequencies. Although, in the same papers, they also claimed that the differences in the dispersive growth kinetics of ZPH found between these cell lines might reflect the differences in the structural heterogeneity of the cell lines. This may not be the case for the current study since the tissue samples themselves have heterogeneous cell compositions.

Taking these earlier successes in cell experiments which used MF680 for distinguishing cellular matrices into account, mitochondria remained the leading candidate as the organelle of choice for several reasons. First, recent research on mitochondria has shown that the mitochondria of cancerous tissues [13] exhibit numerous differences from that of normal tissues. Examples of this include a difference in the membrane potential $\left(\Delta \Psi_{\mathrm{m}}\right)$ generated by mitochondria and morphological differences in shape and size [14,15]. 
Furthermore, lipophilic cationic dye molecules in the rhodamine/rosamine family of dyes have been found to bind preferentially to mitochondria [16-20], which can be attributable to its composition (namely lipids, with a negative-inside membrane potential relative to the cytoplasm). The staining specificity of the rhodamine dyes has been ascertained with several fluorescence and confocal microscopy studies [16-18,21-23]. Among these rhodamine dyes. MF680 has an additional advantage over others because of its red/far red excitation and emission bands. This is particularly important for in vitro tissue samples so as to avoid the range of autofluorescence, as well as the absorption/emission ranges of $\mathrm{pH}$ indicator in the tissue culture media entrapped in the intercellular matrices. Moreover, it is desirable for the dye molecule to exhibit not only a high fluorescence quantum yield but also an efficient NPHB. Despite the difficulty of predicting the NPHB efficiency for any given molecule in a given host, previous work by this group on ionic dye molecules [24], similar to the rhodamine family of dyes, predicted a favorable outcome for this requirement.

Early diagnosis of cancer is crucial to a patient's survival. Patients with early stage cancer confined to a small area have much better prognosis after treatment than patients with more advanced cancers that have spread though out the body [25]. Since early stage cancers are small, they are not visible by the naked eye and therefore molecular imaging techniques could enhance the capability to identify small numbers of cancerous cells within a field of normal cells. These detection techniques potentially could also be used to monitor the response of a patient's tumor to treatment. One characteristic of cancer cells that can be used to differentiate them from normal cells is their increased metabolism [26]. Since mitochondria are critical components of eukaryotic cellular metabolism and are increasingly active as cancer progresses [27], molecules specifically taken up by the mitochondria could 
potentially be used to identify cells with increased mitochondrial activity suggestive of precancerous or cancerous conditions. Mitochondria are the primary site of oxygen metabolism in the cell and mitochondrial dysfunction is associated with cancer [28]. The inner mitochondrial membrane is the site of electron transport in oxidative metabolism. Agents which depolarize mitochondria membrane potential $\left(\Delta \Psi_{\mathrm{m}}\right)$ induce an intrinsic cellular pathway leading to a form of cell suicide called apoptosis [29].

Research presented here is a continuation, refinement, and expansion of previous cell research, regarding the possible future clinical application of differentiating in vitro tissues. Specifically, we present spectral hole burning results for tissues stained with a mitochondrion specific dye, MF680. In this work, fresh, not snap-frozen, tissues were chosen in order to preserve the native intracellular structures, morphological state, and mitochondrion respiration for the organelle specific dye. Three types of minced tissue samples were incubated with MF680: a cancerous ovarian tissue (an adenocarcinoma of ovarian origin, stage $3 \mathrm{C}$ ), a near normal (=proximal to cancerous tissue) peritoneal tissue, and a far normal (=distal location to cancerous tissue) peritoneal tissue. The analogous normal tissue samples were classified depending on the physical distance from the malignant tissue during surgery. With regard to ZPH widths and mean phonon frequencies of side band holes, no significant differences were detected. Differences in hole growth kinetics were also too slight to elucidate differences in cancerous/normal tissues. The Stark measurements, on the other hand, revealed significant differences allowing us to further attempt to use NPHB as an imaging technique for subcellular anomalies of in vitro tissue samples. Potentially, this could even be useful for investigating the mitochondrial membrane potential of tissues. 


\section{Experimental}

\section{Tissue extraction}

Prior to surgery, the patient volunteer consented to a protocol that was approved by the Institutional Review Boards (IRBs) for Research Involving Human Subjects of the University of Oklahoma and Iowa State University. Three labeled containers filled with sterile Minimal Essential Media (MEM) supplemented with 10\% fetal bovine serum and an antibiotics/antimycotic mixture were given to the surgeon. During the course of the operation, the surgeon removed two $1 \mathrm{~cm}$ specimens of normal tissue from the proximal area of the cancer and placed them in the containers. One specimen was taken adjacent to the cancer and another further away. The cancerous tissue was also removed and evaluated by a pathologist who removed a $1 \mathrm{~cm}$ specimen and placed it into the third tube. The tubes were placed immediately on ice and shipped to Iowa State University. Sample preparation

All three types of tissues, minced by surgical blades to an average of $\sim 1 \mathrm{~mm}^{3}$, were stained in suspension using MF680 at a concentration of $250 \mathrm{nM}$ for $15 \mathrm{~min}$ at $37^{\circ} \mathrm{C}$. MEM supplemented with $15 \% \mathrm{FBS}$, penicillin/streptomycin (100 U/mL and $100 \mu \mathrm{g} / \mathrm{mL}$, respectively), and L-glutamine ( $2 \mathrm{mM})$ were chosen as the staining medium, to furnish comparable staining conditions to the prior cell experiments. MF680 was initially dissolved in DMSO. At concentrations used for staining (after diluting with phosphate buffered saline (PBS) and subsequently MEM), the DMSO component of the staining medium was negligible $(\sim 0.001 \%)$. After the $15 \mathrm{~min}$ incubation in suspension the staining media was removed, and the minced tissues were washed (at $100 \times \mathrm{g}$ for $5 \mathrm{~min}$ ) three times with PBS. The tissue fragments were then resuspended in a freezing medium which consisted of MEM 
with $5 \%$ DMSO. Aliquots of these samples were transferred to $5 \mathrm{~mm}$ o.d. gelatin capsules. The samples were then slow-cooled to $-70^{\circ} \mathrm{C}$ overnight in a Nalgene Cryo $1{ }^{\circ} \mathrm{C}$ freezing container to avoid ice formation in the cells at cryogenic temperatures [30,31], where cell viability was tested with the aforementioned cell lines and found to be $\geq 90 \%$ after thawing [11]. Capsules were mounted and rapidly plunged into helium for all hole burning studies. Unless otherwise noted, all chemicals were purchased from Sigma, St. Louis, MO.

Laser and cryostat system

The fluorescence excitation system used has been described previously. Briefly, a Coherent Innova 90-6 argon ion laser (Coherent, Santa Clara, CA) was used to pump a CR 699-29 ring dye laser, using LD688 laser dye (Exciton, Dayton, OH). This laser system provides $100-500 \mathrm{~mW}$ (with $5 \mathrm{~W}$ pumping) power over a wavelength range of $660-720 \mathrm{~nm}$. Laser intensity was stabilized with an LSO0 laser power stabilizer (Cambridge Research and Instrumentation, Cambridge, MA), and subsequently the laser beam was expanded with a telescope. The laser was operated in two modes: broad-range continuous scanning without intracavity etalons, and short-range scanning with intracavity etalons. The scanning ranges and linewidths for the broad scanning mode and short-range mode were $1000 \mathrm{~cm}^{-1}$ and 0.1 $\mathrm{cm}^{-1}$, and $<1.5 \mathrm{~cm}^{-1}$ and $<0.0003 \mathrm{~cm}^{-1}(<10 \mathrm{MHz})$, respectively. Laser polarization was controlled with a polarizer.

Hole burning and scanning intensities were varied by using a series of neutral density absorption filters. Laser beam intensities used for scanning were chosen so as not to further alter the spectra. For kinetics curve detection, burn intensities less than $0.4 \mu \mathrm{W} / \mathrm{cm}^{2}$ were used, while a decrease in the fluorescence signal was concurrently recorded. Because the fluorescence signal decreases very rapidly during the first few seconds of burning, and much 
slower later, the typical channel time for the first $30 \mathrm{~s}$ was $0.1 \mathrm{~s}$. After $30 \mathrm{~s}$, channel collection time was increased to $1 \mathrm{~s}$.

The fluorescence excitation signal was collected with a GaAs photomultiplier tube (PMT) (C659-71; Hamamatsu Photonics, Japan) and photon counter (SR-400; Stanford Research, Sunnyvale, CA). The excitation signal was filtered from the PMT with a $720 \mathrm{~mm}$ longpass filter (720ALP; Omega Optical, Brattleboro, VT). Laser scanning and data collection were done with a PC, controlled by in-house software.

Confocal microscopy

All confocal and time-lapse imaging was performed at the Roy J. Carver Laboratory for Ultra-high Resolution Biological Microscopy (Iowa State University, Ames, IA). The microscope utilized for both imaging methods was a Nikon Eclipse TE200 with an inverted $60 \times$ oil-ommersion lens (NA: 1.40 ; Theoretical Resolution at $632.8 \mathrm{~nm}: 0.276 \mu \mathrm{m}$ ). The confocal instrumentation was manufactured by Prairie Technologies, LLC (Middleton, WI). Confocal imaging was performed with $\mathrm{He}-\mathrm{Ne}(632.8 \mathrm{~nm})$ laser for excitation, with photomultiplier tube (PMT) detection, and a pinhole aperture set to $100 \mu \mathrm{m}$. All software to control the hardware was by Prairie Technologies utilizing National Instruments LabView 5.1.

Tissue section preparation was performed at Histopathology Laboratory, Dept. of Veterinary Pathology (Iowa State University, Ames, IA). As a routine method, bulk tissue fragments (sized $\sim 0.5 \mathrm{~cm}^{3}$ ) of the same ovarian tissue sample, as were utilized for holeburning studies, were frozen within Tissue-Tek O.C.T compound (Sakura Finetek, Torrance, $\mathrm{CA}$ ) down to $77 \mathrm{~K}$ and kept this way until slicing. Cryomicrotomically sliced unfixed tissue sections ( 15 or $30 \mu \mathrm{m}$ ) were mounted on pole-L-lysine (Sigma, St. Louis, MO) treated slides 
for staining and imaging. To facilitate multiple stainings without removing the sample from the microscopy, an in-house culture well (vol. $\sim 0.5 \mathrm{~mL}$ ) was created and affixed to the glass slides. After pre-staining incubation at $37^{\circ} \mathrm{C}$ in culture media for thawing, the slices were stained with MF680 again at a concentration of $250 \mathrm{nM}$ for $15 \mathrm{~min}$ at $37^{\circ} \mathrm{C}$. All images were collected within $1 \mathrm{~h}$ of preparation.

\section{Results and Discussion}

Fluorescence excitation spectra for MF680-stained cancerous (curve c) and normal tissues (curve $\mathrm{a}$ and $\mathrm{b}$ ) at $5.2 \mathrm{~K}$ are given in Fig. 1(A). The figure also shows the fluorescence excitation spectra of MF680 in hyperquenced glassy water (HGW) (curve d). In comparing the spectra of MF680 in HGW with that in tissues, it is apparent that there is a large shift in the fluorescence excitation maxima of the spectra, as was observed in previous cell studies [9]. Although the exact cause of these shifts was not a major focus of this study, it is interesting to note the possible sources of these shifts. The controlled environment of Fig. 1(A) (HGW) has no lipid nature whatsoever; in each tissue sample, however, the dye molecule is located in a lipid-rich environment common to mitochondria. Note that the absorption and emission maxima in ethanol were measured at $682 \mathrm{~nm}$ and $702 \mathrm{~nm}$ [32], respectively. Another factor was elucidated in the work presented by Sakanoue et al. [33] who found that on isolated rat liver mitochondria, the MF680 dye molecule displayed spectral changes dependent on $\Delta \Psi_{m}$. The authors made no attempt to explain the shifts, but it is apparent that this molecule's fluorescence excitation is inherently sensitive to $\Delta \Psi_{\mathrm{m}}$ and/or its host matrix composition. The possibility that the shift is the result of molecular modifications made to the dye in vivo can be discounted, as the rat liver mitochondria study 

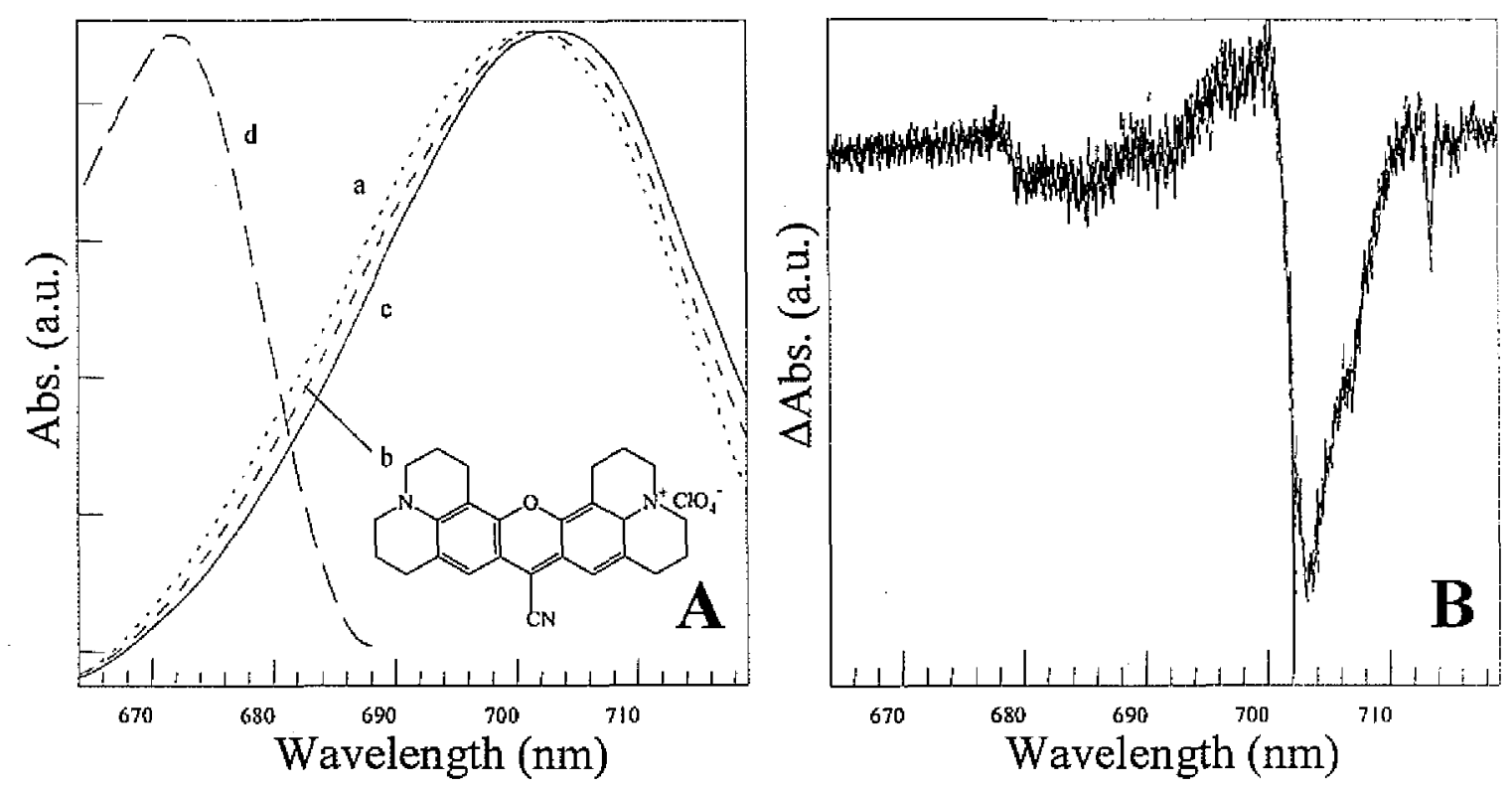

Figure 1 (A) Fluorescence excitation spectra of MF680 in three types: far normal (dotted line; curve a), near normal (dashed line; curve b), and cancerous (solid line; curve c) of tissue measured at $5.2 \mathrm{~K}$, in comparison to the fluorescence excitation spectrum of MF680 in HGW (99.9:0.1 (v/v) water/DMSO) (curve d). Note that all the spectra presented were smoothed for the clarification purpose. Peaks are located at $\sim 702 \mathrm{~nm}, \sim 701 \mathrm{~nm}$, and $\sim 700 \mathrm{~nm}$, for the cancerous, near normal, and far normal samples, respectively. (B) Representative satellite hole structures of MF680 in the tissue samples burned at $702 \mathrm{~nm}$ at $5 \mathrm{~K}$. PSBH is roughly at $24 \mathrm{~cm}^{-1}$ apart from the $\mathrm{ZPH}$ and the most noticeable vibronic peaks on both sides are at $\sim 227 \mathrm{~cm}^{-1}$ from the $\mathrm{ZPH}$. Moreover, the $372 \mathrm{~cm}^{-1}$ mode and the $457 \mathrm{~cm}^{-1}$ mode (possibly the overtone of the above-mentioned vibronic mode) can be seen from the spectra. As described in the text, no significant difference was observed in the satellite hole structures of these tissue samples. 
presented data indicating that the reversibility of fluorescence changes based on perturbations of $\Delta \Psi_{\mathrm{m}}$. This indicates the molecular structure is not being changed. Shown in part (B) of Fig. 1 are representative saturated holes of each tissue sample burned at $702 \mathrm{~nm}$, created with fluences of $15 \mathrm{~J} / \mathrm{cm}^{2}$. The sharp feature coincident with the burn frequency is the zerophonon hole ( $\mathrm{ZPH})$. To the lower energy side of the $\mathrm{ZPH}$ are pseudo-phonon sideband holes (PSBH). The PSBH is observed to be $24 \pm 4 \mathrm{~cm}^{-1}$ for each sample and no significant difference was found in the vibronic satellite hole structures of these samples. Although not significantly different between the tissue samples, the large separation between the ZPH and the PSBH translates into favorable conditions for the observation of hole growth kinetics for the formation of ZPHs with, in theory, little or no interference from the PSBH. The quantitatively smaller value for the separation compared to that of the cell results [8] (30 \pm 3

$\mathrm{cm}^{-1}$ ) was most likely caused by the saturation of the hole (in order to determine satellite hole structures).

In order to confirm the predicted distribution of burning species within the absorption band and the homogeneity of the spectral hole profiles, the action spectra of MF680 in the tissue was measured in 1-nm intervals. The fractional hole depths of action spectra, measured with cancerous tissue, are graphed in Fig. 2, as well as their widths. When hole burning is initiated at low energies and subsequent holes are burned at high energies (i.e. red to blue), the ability to accurately measure hole depths is greatly diminished [34] for the laserinduced hole-filling. Because of this, these holes were burned at higher energies with subsequent holes being burned at lower energies (i.e. blue to red). As seen in the figure, attempts to burn holes in the higher energy side of the band are unsuccessful because of the absorption being increasingly dominated by vibronic/phononic transitions rather than the 


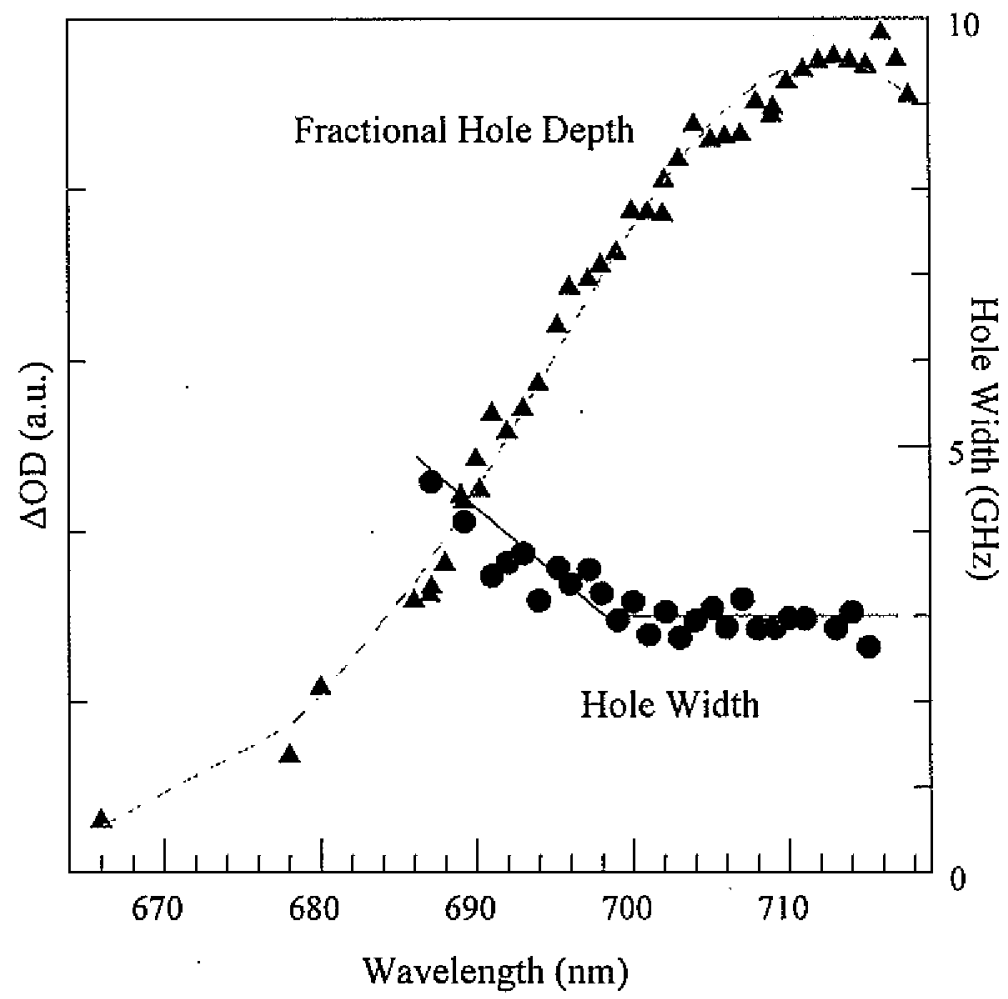

Figure 2 Fractional hole depths and their widths of action spectra of MF680 in cancerous tissue. The dashed line is the Gaussian fit of the fractional hole depth values. Its peak is at $\sim 712.5 \mathrm{~nm}$. 
pure electronic transition, which resembles the action spectra for cell lines. The Gaussian fit of the fractional hole depths gives a peak of $712.5 \pm 0.5 \mathrm{~nm}$, and the hole widths show constant values for burn wavelengths $>698 \mathrm{~nm}$. From those facts, wavelengths around 712 $\mathrm{nm}$ were chosen for hole growth kinetics and Stark hole burning studies discussed later-on.

The dispersive kinetics of hole burning for MF680 in tissues are shown in Fig. 3 at two different burn wavelengths $\left(\lambda_{\mathrm{B}}\right)$. For both, the nonexponential hole burning is evidence that the kinetics cannot be described by a single rate. Treating the kinetics as being described by a Gaussian distribution of rates has been successful for a variety of glassy samples [5,3537]. Experimentally obtained kinetic curves were fit using [38]:

$$
D(t)=\int d \lambda f(\lambda) \int d \alpha \sin \alpha \cos ^{2} \alpha\left(e^{-P_{\theta} P_{T} \alpha(\lambda) \cos ^{2} \alpha}\right),
$$

where $1-D(t)$ is the time-dependent fractional hole depth following a burn for time $t$ at a burn $\omega_{\mathrm{B}} . P$ is the burn photon flux and was determined from the laser power, taking into consideration the beam size at the sample and attenuating filters in the beam path. $\alpha$ is the angle between the laser polarization and the molecular transition dipole, and $f(\lambda)$ is the Gaussian distribution function of tunneling parameter $\lambda$ for the extrinsic two-level systems $\left(\mathrm{TLS}_{\mathrm{ext}}\right)$ active in hole burning. The unitless parameter $\lambda_{0}$ is the distribution center with a standard deviation $\sigma_{\lambda}$. Note that the parameters $\lambda$ and $\alpha$ each define a distribution of hole burning rates that lead to dispersive kinetics, with the $\lambda$ distribution being the dominant factor [7] accounting for the first $80 \%$ of a burn. The parameter $\sigma_{L \mathrm{P}}^{\mathrm{p}}$ is the peak absorption cross-section, where $2.8 \times 10^{-12} \mathrm{~cm}^{2}$, a previously determined [8] value for MF680 in cells. The average quantum yield for hole burning, $\phi$, is given by [39]:

$$
\phi(\lambda)=\frac{\Omega_{0} \exp (-2 \lambda)}{\Omega_{0} \exp (-2 \lambda)+\tau_{\mathfrak{1}}^{-1}} \approx \frac{\Omega_{0} \exp (-2 \lambda)}{\tau_{\mathfrak{n}}^{-1}},
$$


(a)

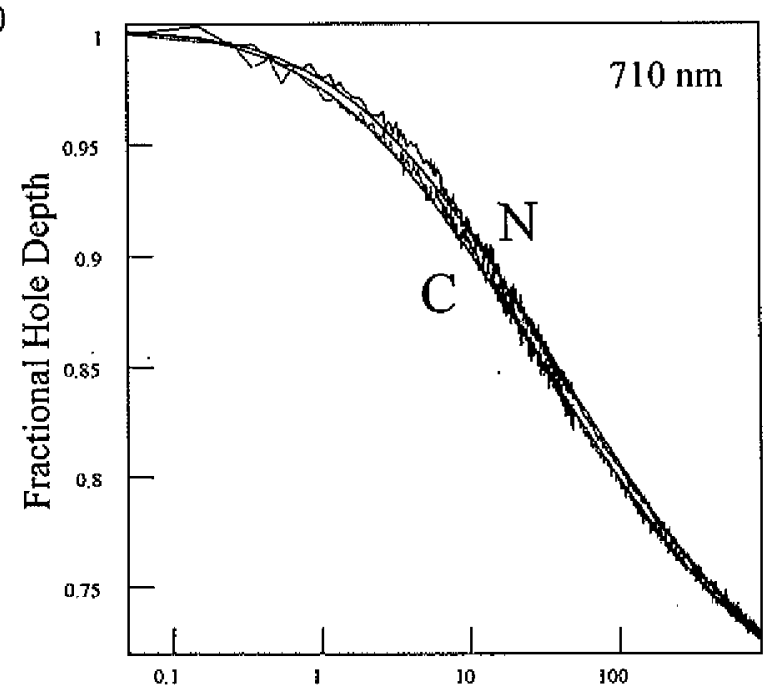

(b)

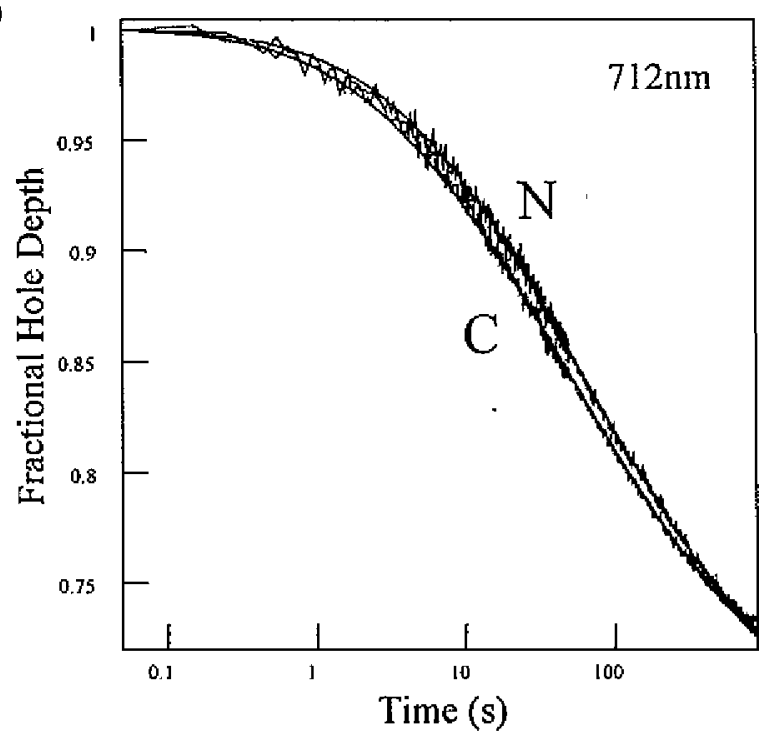

Figure 3 Hole growth kinetics for MF680 in cancerous (C) and far normal (N) tissues at (a) $\lambda_{\mathrm{B}}$ of $710 \mathrm{~nm}$ and at (b) $\lambda_{\mathrm{B}}$ of $712 \mathrm{~nm}$ at $1.8 \mathrm{~K}$. 
where $\Omega_{0} \exp (-2 \lambda)$ represents the phonon-assisted tunneling relaxation rate, with $\Omega_{0}$ set to 7.6 $\times 10^{12} \mathrm{~s}^{-1}[40]$ and $\tau_{\mathrm{f}}$ is the fluorescence lifetime, again previously determined to be $1.8 \mathrm{~ns}$ [8] in aqueous solution at $77 \mathrm{~K}$. A quantum mechanical expression for $\Omega_{0}$ can be found in Ref. [35]. The data in Fig. 3 was fit to Eq. 1 by using a three-parameter least-squares fit. The three fit parameters, $\lambda_{0} ; \sigma_{\lambda} ;$ and the amplitude at $t=0$ (a technical parameter), were used to obtain the fits in the figure. For the fits shown, the Huang-Rhys factor, $S$ was set equal to 1.15, based on independent measurements of saturated hole depths, where in theory the maximum depth of the saturated $\mathrm{ZPH}$ equals $\exp (-S)$. In the fitting procedure, the value of $S$ determines the limiting value of $D(t)$. Values for $\lambda_{0}$ and $\sigma_{\lambda}$ are summarized in Table 1 . The values for the two cellular systems are quite similar, though normal tissue samples have slightly higher values for both $\lambda_{0}$ and $\sigma_{h}$, which is a qualitatively comparable observation to the earlier cell line results [8]. The tendency for smaller $\lambda_{0}$ and $\sigma_{\lambda}$ values to reveal themselves in HGK burned at a lower energy point within each sample, also resembles the cell results.

The effects of a $6.0 \mathrm{kV} / \mathrm{cm}$ Stark field on the $\mathrm{ZPH}$ of MF 680 in cancerous and normal tissues at $\lambda_{B}=710 \mathrm{~nm}$ are shown in Fig. 4 for both parallel (frames (a) and (c)) and perpendicular (frames (b) and (d)) orientations of the burn laser polarization relative to the Stark field. For all tissue samples, only Stark broadening was observed for parallel and perpendicular orientations, and no Stark splitting was observed for either orientation. Broadening effects were found to be reversible when the electric field was turned off, thus returning the holes to their original widths while hole depths decreased slightly owing to spontaneous hole filling [36]. 
Table 1. Dispersive Hole-Growth Kinetics Fit Parameters for Tissues in Comparison to These for Cell Lines ${ }^{a}$

\begin{tabular}{|c|l|c|c|c|c|}
\hline$\lambda_{\mathrm{B}}(\mathrm{nm})$ & & $\lambda_{0}(\mathrm{C})$ & $\lambda_{0}(\mathrm{~N})$ & $\sigma_{\lambda}(\mathrm{C})$ & $\sigma_{\lambda}(\mathrm{N})$ \\
\hline \multirow{2}{*}{710} & Tissue $^{\mathrm{n}}$ & $7.05 \pm 0.10$ & $7.18 \pm 0.10$ & $1.09 \pm 0.10$ & $1.14 \pm 0.10$ \\
\cline { 2 - 6 } & Cell $^{\mathrm{b}}$ & $7.78 \pm 0.13$ & $8.36 \pm 0.12$ & $0.85 \pm 0.10$ & $1.10 \pm 0.09$ \\
\hline \multirow{2}{*}{712} & Tissue $^{2}$ & $7.02 \pm 0.10$ & $7.17 \pm 0.10$ & $1.09 \pm 0.10$ & $1.10 \pm 0.10$ \\
\cline { 2 - 6 } & Cell $^{\mathrm{b}}$ & $7.84 \pm 0.10$ & $8.36 \pm 0.10$ & $0.68 \pm 0.10$ & $1.15 \pm 0.08$ \\
\hline
\end{tabular}

a From Ref. [8]

${ }^{\mathrm{b}}$ Carcinoma cell line - OV167/Analogous normal cell line - OSE14(tsT)-14 


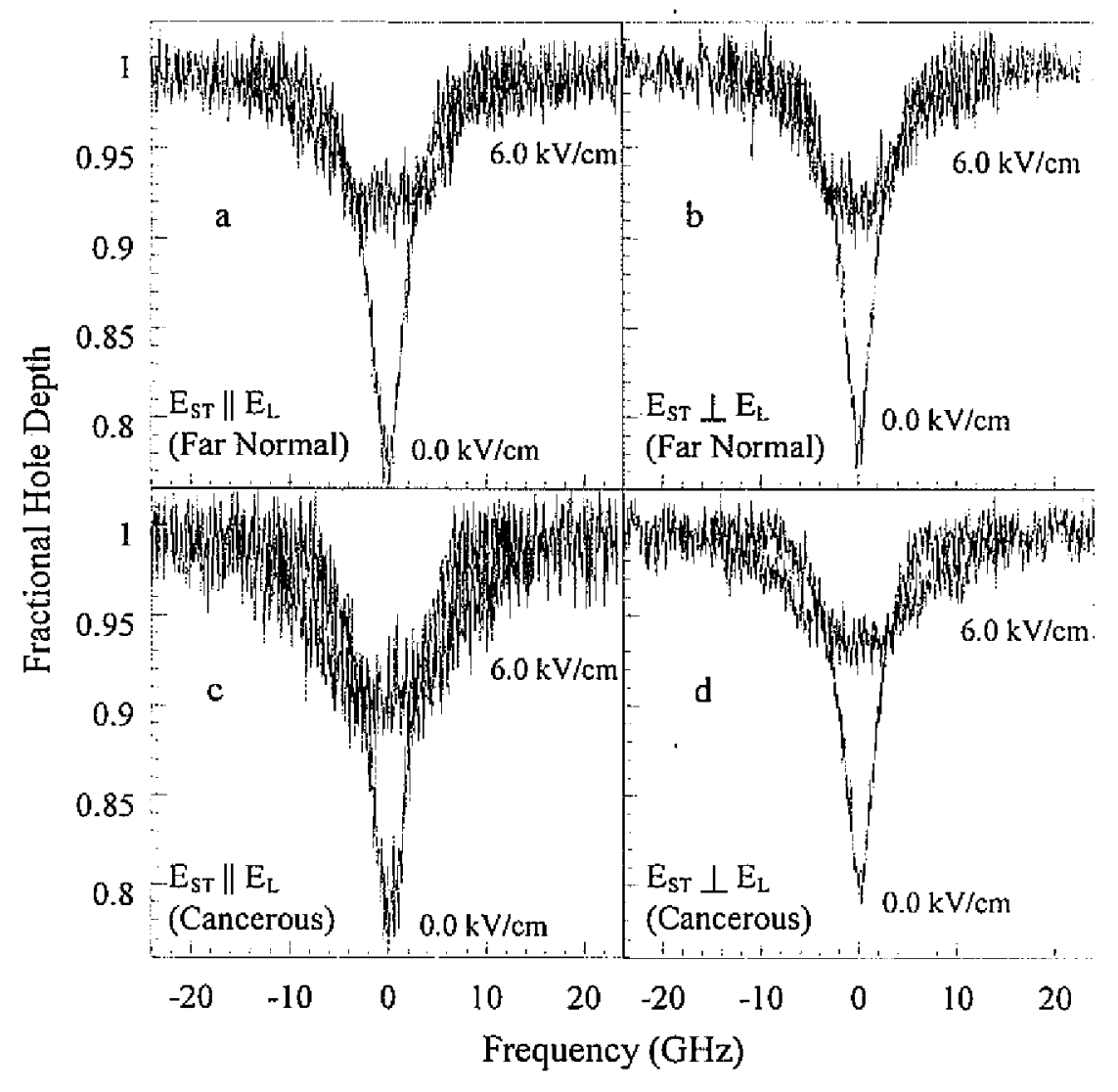

Figure $4 \quad$ ZPH's, burned at $710 \mathrm{~nm}$, at zero-field and with a $6.0 \mathrm{kV} / \mathrm{cm}$ external field for MF680 in a far normal tissue sample (panels $a$ and $b$ ) and in a cancerous tissue sample (panels $\mathrm{c}$ and $\mathrm{d}$ ) at $1.8 \mathrm{~K}$. Panels a and $\mathrm{c}$ correspond to burn laser polarizations parallel to the Stark field, while the panels $b$ and $d$ represent laser polarization perpendicular to the Stark field. 
A more quantitative comparison of the Stark hole broadening for the three types of tissue samples can be made by calculating the change in dipole moment, $\Delta \mu$. Calculations to obtain these values were performed according to Kador et al. [41] and were determined by fitting a plot of hole width, $\Gamma_{\mathrm{h}}$, of the $\mathrm{ZPH}$ as a function of applied electric field strength, according the following equation:

$$
\Gamma_{\mathrm{h}}(\mathrm{F})=\gamma_{0}\left(1+\mathrm{F}^{2}\right)^{1 / 2}
$$

where $\gamma_{0}$ is the width of the ZPH at zero field and includes contributions from both the homogeneous width of the ZPH plus any additional width from spectral diffusion or fluence broadening. $\mathrm{F}$ is defined by

$$
\mathrm{F}=\frac{2 f \Delta \mu \mathrm{E}_{\mathrm{s}}}{h \gamma}
$$

This calculation gives the result for $f \Delta \mu$, where $f$ is the local field correction, which is unknown for the MF680 matrix. Results for these calculations are given in Table 2. An example of this is given in Fig. 5 for cancerous and far normal tissue samples in the parallel orientation. A quantitative comparison of results obtained for the perpendicular polarization was omitted since the values do not exhibit a dependency on the polarization of the applied electric field, as was the case in the results of MF680 in cell lines [9]. From the data in Table 2 , one can see that, indeed, differences in the values of $f \Delta \mu$ exist between the three tissue samples. Of particular interest is the determination that $f \Delta \mu$ s for the normal tissues are lower in value than the cancerous tissue on all four $\lambda_{\mathrm{B}} \mathrm{S}$, most likely a result of the lowered $\Delta \Psi_{\mathrm{m}}$ in normal tissues, vide infra. Also of interest in the table is that the ratios of these dipole moment changes $f \Delta \mu(\mathrm{c}) / f \cdot \Delta \mu(\mathrm{fn})$ is all $\sim 1.35$ on all these burn wavelengths (with an 
Table 2. Electric Field-Induced Permanent Dipole Moment Changes for MF680 in Tissue Samples and Their Ratios

\begin{tabular}{|c|c|c|c|}
\hline$\lambda_{\mathrm{B}}(\mathrm{nm})^{\mathrm{a}}$ & Tissue Type & $f \cdot \Delta \mu(\mathrm{D})^{\mathrm{b}}$ & $f \cdot \Delta \mu(\mathrm{c}) / f \Delta \mu(\mathrm{fn})$ Ratio \\
\hline \multirow{2}{*}{706} & cancer & $2.486 \pm 0.056$ & \\
& near normal & $2.175 \pm 0.028$ & 1.3881 \\
& far normal & $1.791 \pm 0.039$ & \\
\hline \multirow{3}{*}{708} & cancer & $2.327 \pm 0.060$ & \\
& near normal & $2.014 \pm 0.058$ & 1.3529 \\
& far normal & $1.720 \pm 0.068$ & \\
\hline \multirow{3}{*}{710} & cancer & $2.870 \pm 0.033$ & \\
& near normal & $2.416 \pm 0.036$ & 1.3486 \\
& far normal & $2.128 \pm 0.045$ & \\
\hline \multirow{3}{*}{712} & cancer & $2.672 \pm 0.037$ & \\
& near normal & $2.513 \pm 0.047$ & 1.3572 \\
& far normal & $1.969 \pm 0.040$ & \\
\hline
\end{tabular}

${ }^{a}$ All the data presented in the table are measured with the laser polarization parallel to the Stark field $\left(\mathbf{E}_{\mathrm{ST}} \| \mathbf{E}_{\mathrm{L}}\right)$.

${ }^{b}$ Uncertainties are given by the fitting procedures. 


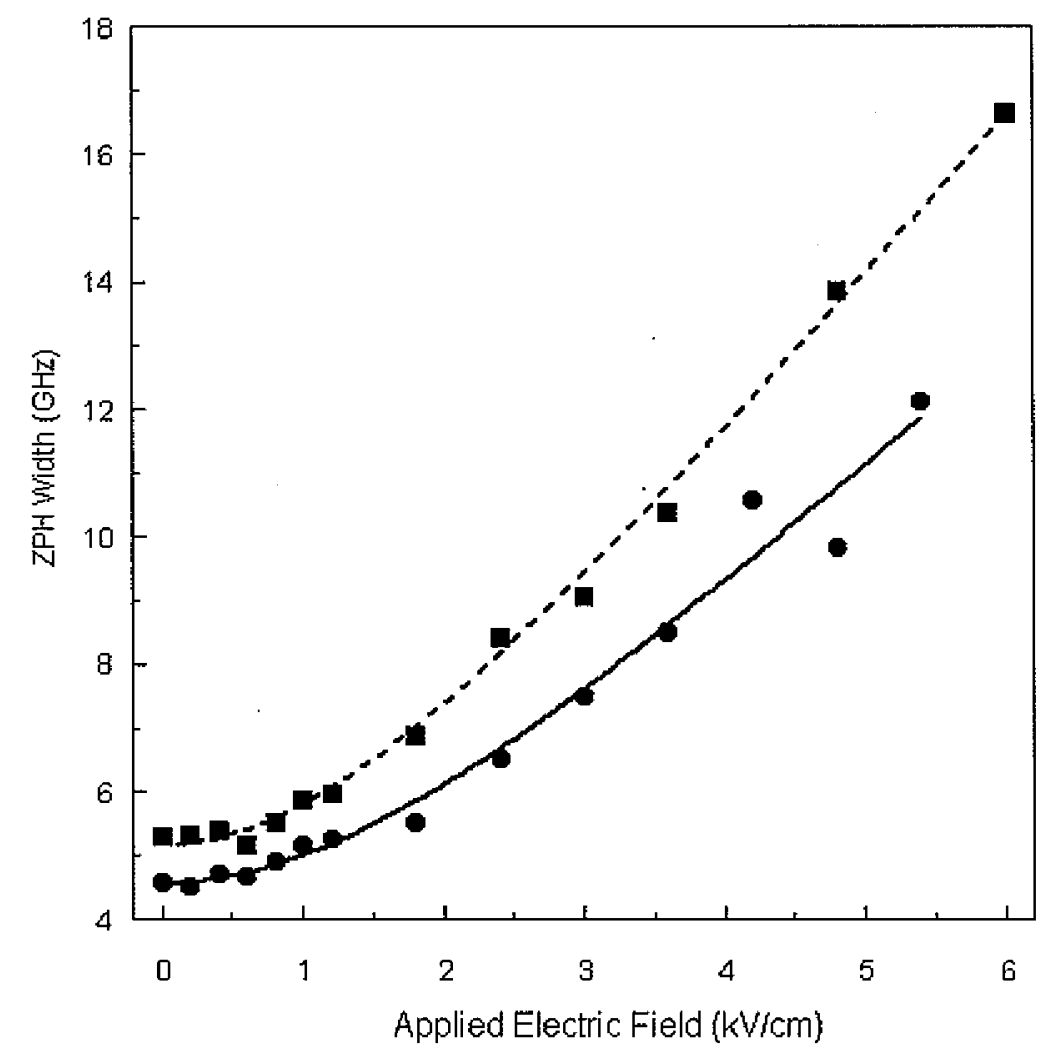

Figure 5 Dependence of hole widths on the strength of the Stark field for MF680 in cancerous (squares) and far normal (circles) tissue samples for a burn laser polarization parallel to the Stark field. Holes were burned at $712 \mathrm{~nm}$.

Theoretical fits of Stark broadening are also given for cancerous (dashed line) and far normal (solid line) tissues. 
exception of the $\lambda_{B}=706 \mathrm{~nm}$ case, which still lies well within the bounds of experimental uncertainty).

To understand these results, it must be considered that the change in dipole moment $\Delta \mu$, is equal to the sum of the intrinsic molecular dipole moment difference, $\Delta \mu_{0}$, and a matrix-induced component, $\Delta \mu_{\text {ind. }}$ For the case where $\Delta \mu_{0} \gg \Delta \mu_{\text {ind, }}$ one would expect to observe Stark splitting for one laser orientation and Stark broadening for the other orientation [42]. However, when Stark broadening occurs at both polarizations, where $\Delta \mu_{\|} \cong \Delta \mu_{\perp}, \Delta \mu_{\mathrm{ind}}$ will dominate, thus indicating $\Delta \mu_{\mathrm{ind}}$ is randomly oriented with respect to the transition dipole of MF680 and the laser polarization. Second, according to Vauthey et al. [43], since $\Delta \mu_{\text {ind, }}$, is composed of a random, $\Delta \mu_{\mathrm{r}}$, and nonrandom, $\Delta \mu_{\mathrm{nr}}$, component, it itself varies in random fashion. This is consistent with the observation that $\Delta \mu_{\mathrm{l}} \cong \Delta \mu_{\perp}$ within each tissue line. Most importantly, these observations conclude that overall $\Delta \mu$ in the present case is due to matrixinduced factors, and our observations can be explained by the in vivo interaction of the dye molecule with the mitochondrial matrix.

A confocal microscope image of the cryomicrotomically sliced cancerous tissue sample stained with $250 \mathrm{nM}$ of MF680 is presented in Fig. 6 . One notable feature of the image is the specific highlights of the dye in structured organelles, which most closely resemble mitochondria when referenced to other studies dealing with the staining of $i n$ situ mitochondria [17]. To rule out the possibility that the MF680 dye was locating preferentially elsewhere than the mitochondria, or even entrapped in the intercellular matrices with this concentration, the sodium azide test described in Ref. [8] was conducted. Briefly, a small amount of sodium azide was added to the sample in PBS while being imaged on the microscope; within several seconds, the fluorescence intensity of the cells which comprised 
107.

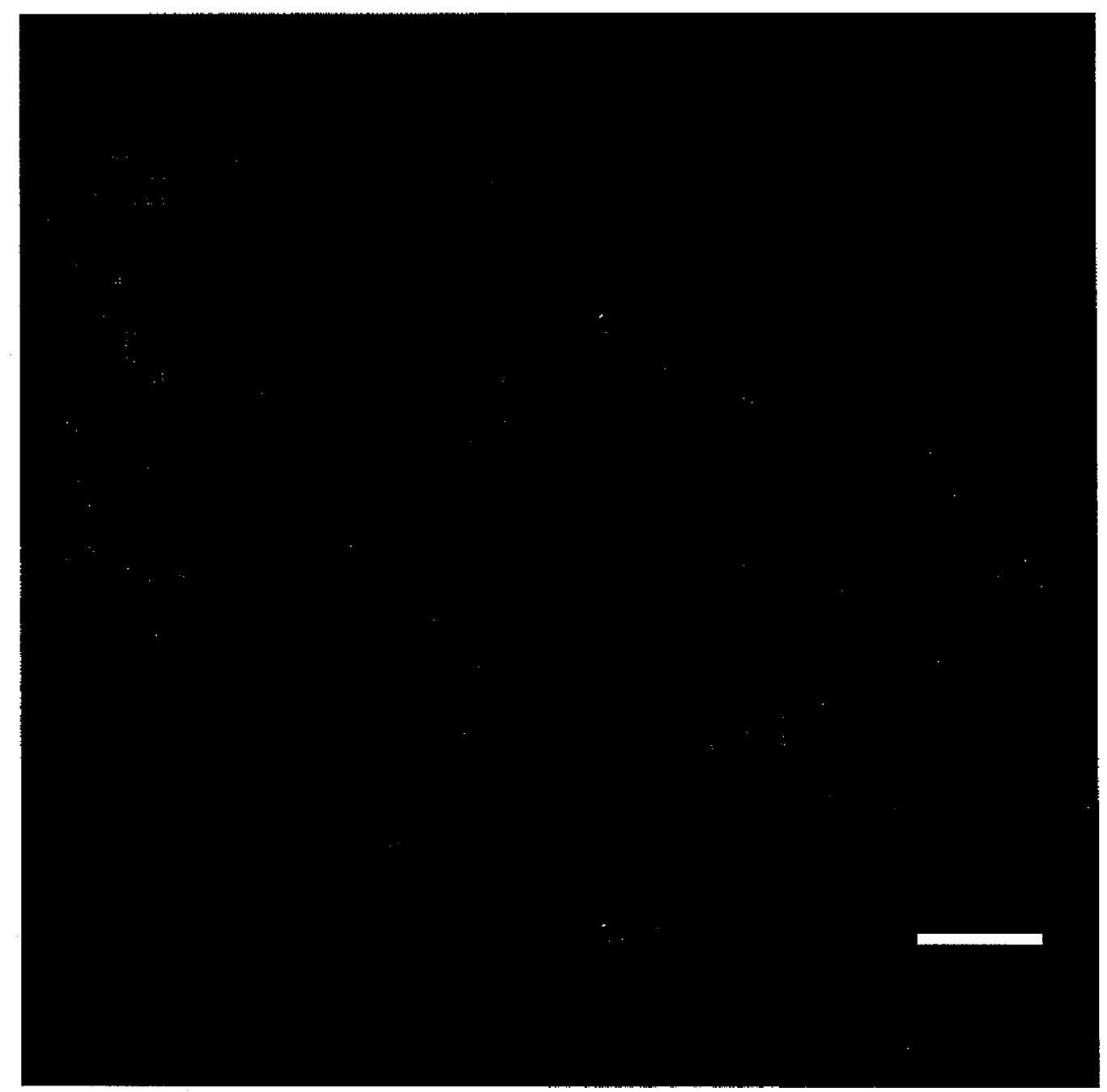

Figure 6 A confocal image of a $\sim 30 \mu \mathrm{m}$ thick cryomicrotomically sliced cancerous tissue sample stained with $250 \mathrm{nM}$ MF680 for $15 \mathrm{~min}$. The white bar represents $20 \mu \mathrm{m}$. 
the tissue was observed to diminish (data not presented). Sodium azide is a toxin which targets enzyme complex IV in the mitochondrial electron transport chain, supposedly resulting in deterioration of the mitochondrial membrane potential. Since the MF680 dye is cationic and requires the membrane potential to maintain its position within mitochondrial membranes, this observation was the direct result of the MF680 being released by the mitochondria to levels below detection due to the influence of the sodium azide.

A parameter we have not elaborated on this point is $\Delta \Psi_{\mathrm{m}}$. It has been observed in previous studies by other groups $[16,18-20,44]$ to be greater in magnitude in carcinoma cells/tissues relative to normal cells/tissues. Although the reported values differ $(100 \mathrm{mV} \leq$ $\Delta \Psi_{\mathrm{m}} \leq 200 \mathrm{mV}$ ) depending on the cell lines or origin of the tissue, there is an approximate 60-90 mV range difference between reported comparative carcinoma and normal cell cultures. A ratio based on this difference gives a value of $\sim 1.5$, where the ratio between $f \cdot \Delta \mu$ values measured for preliminary cell experiments also gave a value of $\sim 1.5$ at $\lambda_{B}$ of 711.8 and $711.6 \mathrm{~nm}$ [9]. The ratio obtained for this tissue study gives $\sim 1.35$, which is indeed quantitatively smaller though it is certainly a significant difference while considering the inhomogeneity of cell populations which comprise the tissue can easily make the difference inarticulate. Although further experimentation is necessary to expand on the correlation and to quantificate the differences, it is interesting to note the apparent relation between the ratios.

In summary, we are encouraged by our findings regarding the qualitative as well as quantitative differences found between the three types of tissue in the series of experiments utilizing the Stark effect. The difference in the tunneling parameters given by the hole growth kinetics between the samples was small and, unlike the earlier cultured cell study, 
haven't shown the ability to distinguish tissue cellular matrices surrounding the

chromophores. Though, this can be understandable as the tissue samples are comprised of inhomogeneous cell populations, while the cell experiment was performed for supposedly two distinctly different, homogeneous (in terms of cellular matrices, not proliferation) cultured cell lines. We anticipate that additional studies targeting in vivo mitochondria will allow us to expand these findings for differentiating in vitro tissues.

\section{Acknowledgement}

Ames Laboratory is operated for the U.S. Department of Energy by lowa State University under Contract W-7405-Eng-82. This work was supported by the Office of Biological and Environmental Research. The authors thank Robert Doyle at the ISU Carver Imaging Laboratory for assistance with confocal microscopy instruments.

\section{References}

1. Walsh, C. A., Berg, M., Narasimhan, L. R., Fayer, M. D. J. Chem. Phys., 1987, 86, 77 .

2. Kador, L., Personov, R., Richter, W., Sesselmann, T., Haarer, D. Polymer J., 1987, 19,61 .

3. Van den Berg, R., Völker, S. Chem. Phys., 1988, 128, 257.

4. Silbey, R. J., Koedijk, J. M. A., Völker, S. J. Chem. Phys., 1996, 105, 901.

5. Reinot, T., Hayes, J. M., Small, G. J. J. Chem. Phys, 1997, 106, 457.

6. Reinot, T., Hayes, J. M., Small, G. J. J. Chem. Phys., 1997, 110, 4820.

7. Reinot, T., Small, G. J. J. Chem. Phys., 2001, 114, 9105. 
8. Walsh, R. J., Reinot, T., Hayes, J. M., Kalli, K. R., Hartmann, L. C., Small, G. J. Biophys. J., 2003, 84, 1299.

9. Walsh, R. J., Reinot, T., Hayes, J. M., Kalli, K. R., Hartmann, L. C., Small, G. J. J. Lumin., 2002, 98, 115.

10. Milanovich, N., Rätsep, M., Reinot, T., Hayes, J. M., Small, G. J. J. Phys. Chem. B, $1998,102,4265$.

11. Milanovich, N., Reinot, T., Hayes, J. M., Small, G. J. Biophys. J., 1998, 74, 2680.

12. Furusawa, A., Suga, T., Kiyoshi, U. J. Opt. Soc. Am. B., 1994, 11, 1456.

13. Yaffe, M. P. Science, 1999, 283, 1493.

14. Djaldetti, M. Acta Haematol., 1982, 68, 241.

15. Eapen, C. E., Madesh, M., Balasubramanian, K. A., Pulimood, A., Mathan, M., Ramakrishna, B. S., Scand. J. Gastroenterol., 1998, 33, 975.

16. Johnson, L. V., Walsh, M. L., Chen, L. B. Proc. Natl. Acad. Sci. USA, 1980, 77, 990.

17. Chen, L. B., Summerhayes, I. C., Johnson, L. V., Walsh, M. L., Bernal, S. D., Lampidis, T. J. Cold Spring Harb. Symp. Quant. Biol, , 1982, 46, 141.

18. Summerhayes, I. C., Lampidis, T. J., Bernal, S. D., Nadakavukaren, J. J., Nadakavukaren, K. K., Shepherd, E. L., Chen, L. B. Proc. Natl. Acad. Sci. USA, $1982,79,5292$.

19. Davis, S., Weiss, M. J., Wong, J. R., Lampidis, T. J., Chen, L. B. J. Biol. Chem., $1985,260,13844$.

20. Modica-Napolitano, J. S., Aprille, J. R. Advanced Drug Delivery Reviews, 2001, 49, 63 .

21. Darzynkiewicz, Z., Staiano-Coico, L., Melamed, M. R. Proc. Natl. Acad. Sci. USA, $1981,78,2383$.

22. Leprat, P., Ratinaud, M. H., Julien, R. Mech. Ageing Dev., 1990, 52, 149.

23. Bassøe, C.-F., Li, N., Ragheb, K., Lawler, G., Sturgis, J., Robinson, J. P. Cytometry Part B, 2003, 51B, 21 .

24. Fearey, B. L., Carter, T. P., Small, G. J. J. Phys. Chem., 1983, 87, 3590. 
25. Eltabbakh, G. H. Minerva Ginecologica., 2004, 56(1), 81.

26. Stubbs, M., Robinson, S. P., Hui, C., Price, N. M., Rodrigues, L. M., Howe, F. A., Griffiths, J. R. Advances in Enzyme Regulation, 2002, 42, 131.

27. Chen, Y., Liu, Z. Y., Li, R. X., Guo, Z. Lymphology, 1999, $32(2), 70$.

28. Eng, C., Kiuru, M., Fernandez, M. J., Aaltonen, L. A. Nature Rev. Cancer, 2003, 3, 193.

29. Green, D. R., Reed, J. C. Science, 1998, 281, 1309.

30. Farrant, J. Walter, C. A., Lee, H., Morris, G. J., Clarke, K. J. J. Microsc., 1977, 111 , 17.

31. Franks, F. J. Microsc., 1977, 111, 3 .

32. Haugland, R. P., Handbook of Fluorescent Probes and Research Chemicals ( $9^{\text {lh }}$ edn.), 2002, Molecular Probes, Inc., Eugene, pp. 487.

33. Sakanoue, J., Ichikawa, K., Nomura, Y., Tamura, M. J. Biochem. (Tokyo), 1997, 121. 29.

34. Shu, L., Small, G. J. J. Opt. Soc. Am. B, 1992, 9, 738.

35. Kenney, M. J., Jankowiak, R., Small, G. J. Chem. Phys., 1990, 146, 457.

36. Shu, L., Small, G. J. J. Opt. Soc. Am. B, 1992, 9, 733.

37. Kim, W.-H., Reinot, T., Hayes, J. M., Small, G. J. J. Phys. Chem., 1995, 99, 7300.

38. Reinot, T., Small, G. J. J. Phys. Chem., 2000, 113, 10207.

39. Reinot, T., Kim, W.-H., Hayes, J. M., Small, G. J. J. Opt. Soc. Am. B, 1997, 14, 602.

40. Kim, W.-H., Reinot, T., Hayes, J. M., Small, G. J. J. Chem. Phys., 1996, 104, 6415.

41. Kador, L., Haarer, D., Personov, R. J. Chem. Phys., 1987, 86, 5300.

42. Meixner, A. J., Renn, A., Bucher, S. E., Wild, U. P. J. Phys. Chem., 1986, 90, 6777.

43. Vauthey, E., Holliday, K., Changiang, W., Renn, A., Wild, U. P. Chem. Phys., 1993, 171,253 .

44. Dairkee, S. H., Hackett, A. J. Breast Cancer Res. Treat., 1991, $18,57$. 


\section{CHAPTER 6. GENERAL CONCLUSIONS}

Presented in this dissertation is the successful demonstration that nonphotochemical hole burning (NPHB) imaging can be used to study in vitro tissue cellular systems for discerning differences in cellular ultrastructures due to cancer development. This has been accomplished with the surgically removed cancerous ovarian and analogous normal peritoneal tissues from the same patient and the application of a fluorescent mitochondrion specific dye, Molecular Probe MitoFluor Far Red 680 (MF680), commonly known as rhodamine 800 , that has been proven to exhibit efficient NPHB. From the results presented in Chapters 4 and 5, and Appendix B, the following conclusions were made: 1) fluorescence excitation spectra of MF680 and confocal microscopy images of thin sliced tissues incubated with MF680 confirm the site-specificity of the probe molecules in the cellular systems. 2) Tunneling parameters, $\lambda_{0}$ and $\sigma_{\lambda}$, as well as the standard hole burning parameters (namely, $\gamma$ and S), have been determined for the tissue samples by hole growth kinetics (HGK) analyses. Unlike the preliminary cultured cell studies, these parameters have not shown the ability to distinguish tissue cellular matrices surrounding the chromophores. 3) Effects of an external electric (Stark) field on the nonphotochemical holes have been used to determine the changes in permanent dipole moment $(f \cdot \Delta \mu)$ for MF680 in tissue samples when burn laser polarization is parallel to the Stark field. Differences are detected between $f \cdot \Delta \mu \mathrm{s}$ in the two tissue samples, with the cancerous tissue exhibiting a more pronounced change ( 1.35 -fold increase) in permanent dipole moment change relative to the normal analogs. It is speculated that the difference may be related to differences in mitochondrial membrane potentials in these tissue samples. 4) In the HGK mode, hole burning imaging (HBI) of cells adhered to coverslips and cooled to liquid helium temperatures in the complete absence of cryopreservatives, 
shows the ability to distinguish between carcinoma and analogous normal cells on the singlecell level. In future applications, this system has the potential to be used with smears of tissue samples for single-layer HBI analysis.

These conclusions demonstrate that HBI has the potential of providing detailed information about localized intracellulat environments and for detecting changes in the physical characteristics (e.g., electrical properties) of cells which constitute the in vitro tissue samples. For the latter, the long-term goal will be to develop NPHB into a diagnostic technique for the early detection of cancer by exploiting the physical differences between normal and cancerous cells and tissues. Moreover, because of the aforementioned HBI's capability to detect cellular anomalies, it has the potential of being used in conjunction with studies involving photodynamic therapy, assuming the chromophore is carefully selected.

Now that a working model has been established for ovarian surface tissues and the test probe molecule, MF680, and future work will include finding other probe molecules that are both site-selective and that will hole burn. Note that in the sense of elucidation of differences between cancerous and normal tissues or detection of cellular anomalies, dyes are not necessarily mitochondrion-specific probes (i.e., including probes targeting other organelle or even the structural foundations of cells such as the cytoskeleton, microfilaments, or microtubules). Some commercially available novel mitochondrion-specific dyes have been screened as other potential candidates to affirm the differences observed with MF680, but unfortunately, very few of these satisfy the necessary criteria for use in hole burning experiments on cells. One potential candidate as a probe molecule that is both mitochondrion-specific and will hole burn was found, and that is chloromethyl-X-rosamine (CMXRos) (see Appendix A). Although the preliminary results with this dye appear 
promising, more work, for instance the dye binding site/mode analysis, must be done to further characterize this molecule. In conclusion, the application of hole burning to cellular systems of cells/tissues has great potential as useful tool for basic research in the biological sciences and as a clinical technique. 


\section{APPENDIX A. ANOTHER DYE CANDIDATE: CMXROS}

For the purpose of reaffirming the results obtained from Stark hole burning experiments on the OV167 and VOSE 14 cell lines using the dye MF680, an alternative dye was sought. The dye selected for this purpose was Chloromethyl-X-rosamine (CMXRos or MitoTracker Red; Figure A.1(a)). This dye, developed commercially by Molecular Probes (Eugene, OR), is a mitochondrion-selective probe. Dyes of this type have been used to measure inner mitochondrial transmembrane potentials $\left(\Delta \Psi_{\mathrm{m}}\right)[1]$, photosensitization $[2,3]$, and the relative changes of $\Delta \Psi_{\mathrm{m}}$ in early apoptotic (cell death) processes [4,5]. More recently, it has been increasingly used to monitor $\Delta \Psi_{\mathrm{m}}$ in situ with fluorescence microscopy [6] and cytofluorometric analyses $[5,7,8]$ in addition to more traditional cationic lipophilic fluorochromes such as 3,3'-dihexyloxacarbocyanine iodide $\left(\mathrm{DiOC}_{6}(3)\right)$ [9], rhodamine 123 , tetramethylthodamine methyl ester (TMRM) and 5,5',6,6'-tetrachloro-1,1'3,3'tetraethylbenzimidazolcarbocyanine iodide (JC-1) [10]. The rationale is that the positively charged probe will be accumulated by energized mitochondria in response to $\Delta \Psi_{\mathrm{m}}$, followed by binding with thiols on proteins and peptides via the probe chloromethyl moiety [5].

The biggest advantage of this dye for hole burning imaging is the commercial availability of the non-fluorescent reduced form of CMXRos (CM- $\mathrm{H}_{2} \mathrm{XRos}$ ), which does not fluoresce until it enters actively respirating mitochondria. This enables one to distinguish the bound probe signal from the non-binding dye contribution in the intracellular matrix. In addition, because of its low hole-burning efficiency, it can be applied to single cell hole growth kinetic studies over a longer period of time. On the other hand, since the absorption range ( $-600 \mathrm{~nm}$; see Figure A.1(b)) overlaps with the autofluorescence region, close monitoring of the autofluorescence is well advised. Moreover, the aforementioned binding 
(a)

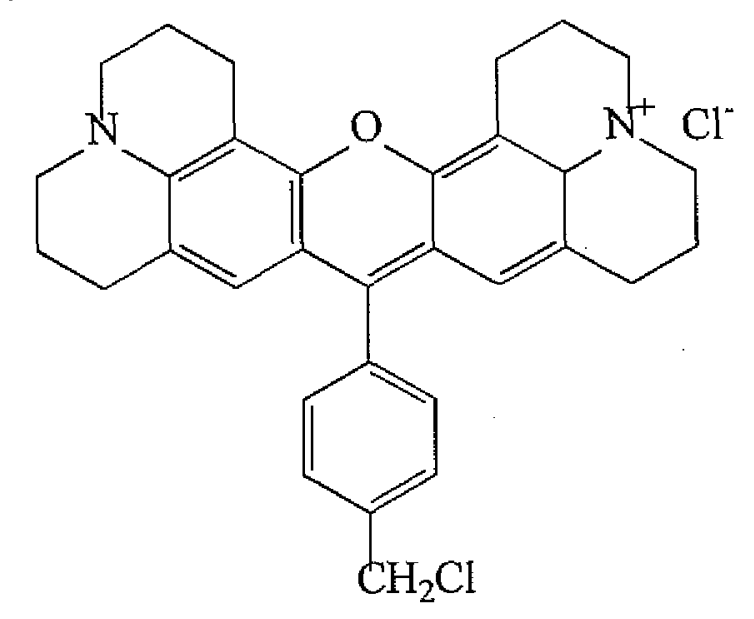

(b)

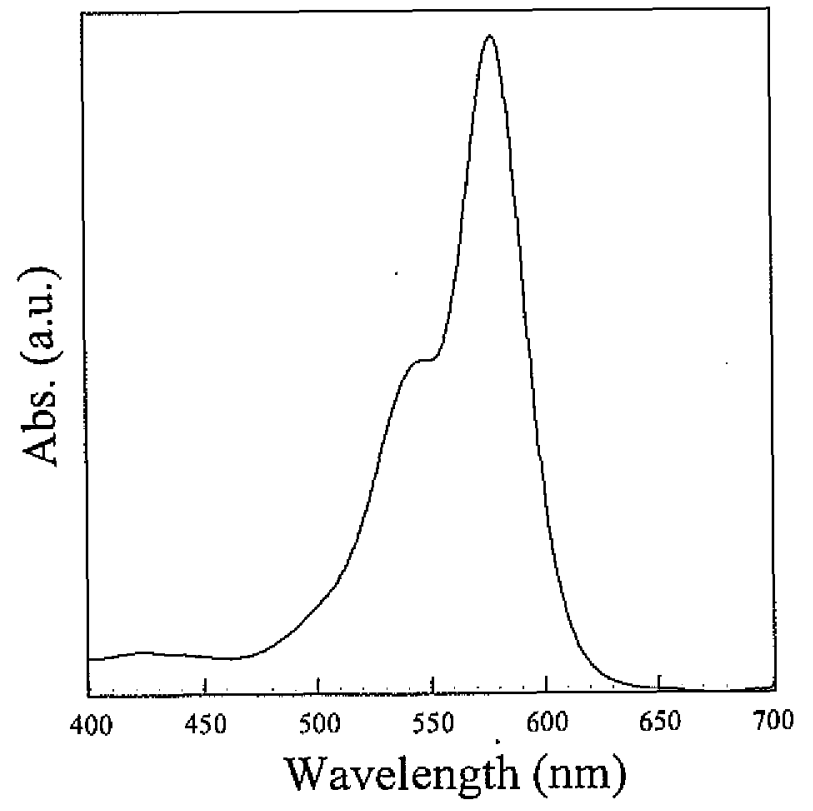

Figure A.1 (a) Molecular structure of CMXRos. Note that replacing the chloromethylphenyl group with a cyano group results in the same structure as MF680, the dye used in the study described in Chapters 4 and 5. (b) Absorption spectrum of CMXRos in 99:1 (v/v) water/DMSO at room temperature, measured with a Varian Cary 10 UV-VIS spectrometer. Maximum absorption is at $578 \mathrm{~nm}$. 
mode of CMXRos to mitochondria at its SH group needs to be considered as well, since the thiol-conjugation is suggested irrespective of the magnitude of $\Delta \Psi_{m}[11]$.

The absorption spectrum of CMXRos in 99:1 (v/v) water/DMSO at room temperature, and the fluorescence excitation spectrum at $5.2 \mathrm{~K}$, are shown in Figures A.1(b) and A.2, respectively. Figure A.2. also depicts the fluorescence excitation spectra of CMXRos in cells along with the fractional hole depths measured in the action spectra obtained at $5.2 \mathrm{~K}$. See the figure caption for the burn fluence data. The cells were stained with $50 \mathrm{nM}$ of CMXRos standing at $37^{\circ} \mathrm{C}$ for $20 \mathrm{~min}$. The hole widths of these action spectra were found to be constant for $\lambda_{\mathrm{B}}>603 \mathrm{~nm}$ (data not shown). This spectral shift pattern (the cell line having the higher $\Delta \Psi_{\mathrm{m}}$ shows a larger red-shift) resembles the fluorescence excitation spectra of MF680 in cells. It should be noted that the peak positions of the band were found to be very sensitive to the morphological condition of the cells, which are altered significantly, for instance, by the cooling rate of the samples. The pre-/post-burn spectra of CMXRos in the OV167 carcinoma cell line burned at $\lambda_{\mathrm{B}}=603.5 \mathrm{~nm}$ and the satellite hole structures are presented in Figures A.3(a) and A.3(b), respectively. As with MF680, the fluorescence lifetime of CMXRos was determined at liquid nitrogen temperature The average fluorescence lifetime was determined to be $2.7 \mathrm{~ns}$ in water (data not shown), which is applied for the hole growth kinetics (HGK) fitting. The zero-fluence hole-width of CMXRos in the cellular matrix was measured at $1.7 \mathrm{GHz}$ at $\mathrm{T}=1.8 \mathrm{~K}$. The peak absorption cross section at $1.8 \mathrm{~K}, \sigma_{\mathrm{LT}}$, which was used to fit HGK curves was also determined, as described previously [12], to be $3.0 \times 10^{-11} \mathrm{~cm}^{2}$. From independent measurements of saturated hole depths, the Huang-Rhys $(S)$ factor was set equal to 1.7 . 


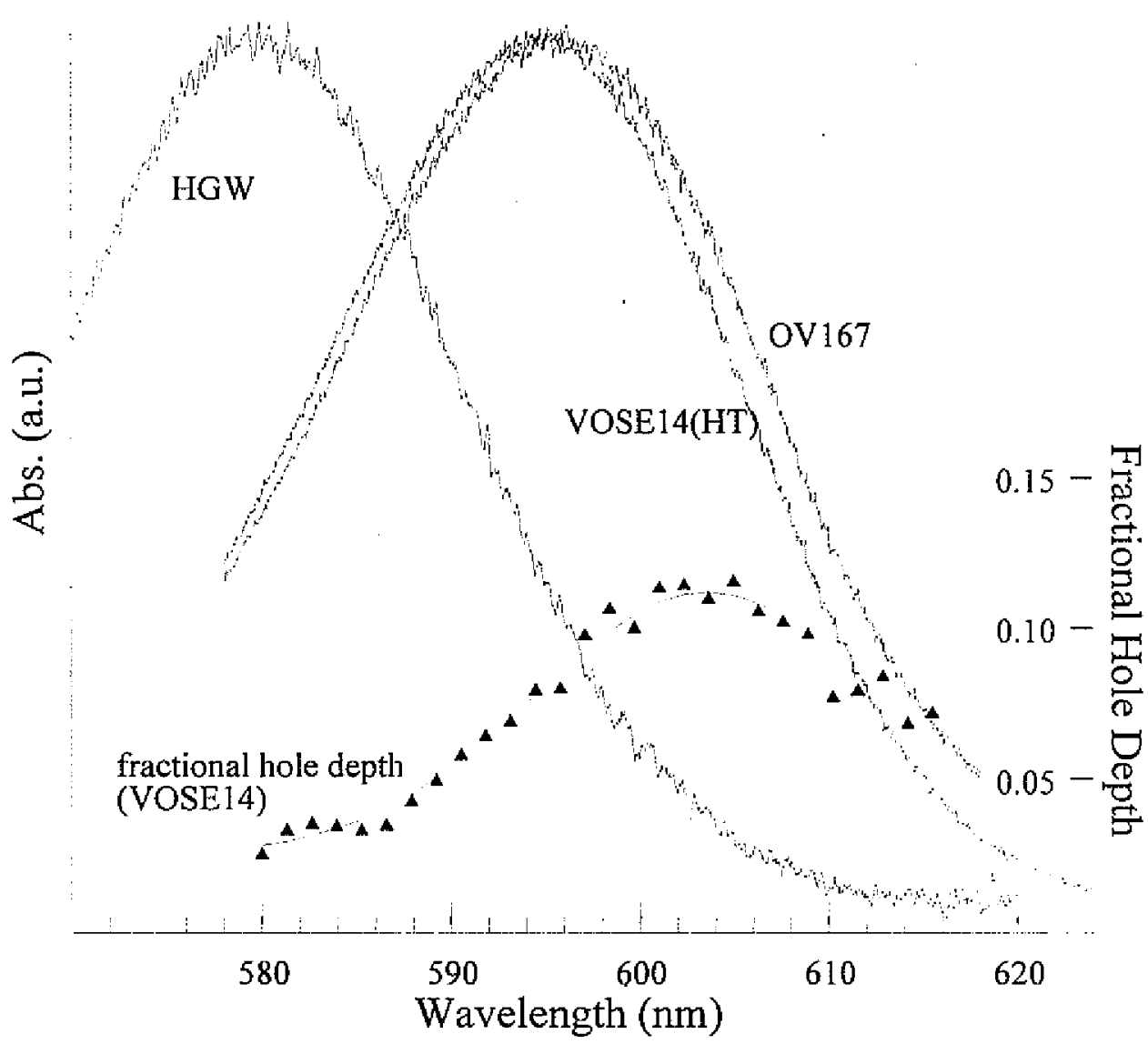

Figure A.2 Fluorescence excitation spectra (at 5.2 K) of CMXRos in HGW, the VOSE14 normal cell line and the OV167 carcinoma cell line. The peaks are measured at $580.2 \mathrm{~nm}, 595.2 \mathrm{~nm}$, and $596.1 \mathrm{~nm}$, respectively. Distribution of fractional hole depths of action spectra measured for VOSE14 at $5.2 \mathrm{~K}$ is also graphed in the same frame, along with a Gaussian fit. Burn intensity and time for the action spectra were $-72.5 \mu \mathrm{W} / \mathrm{cm}^{2}$ and $300 \mathrm{~s}$. The peak of the Gaussian was at $\sim 603.5 \mathrm{~nm}$. The peak position and the width of the Gaussian distribution for OV167 were closely matched to those of VOSE14, despite the shifted absorption peaks. 

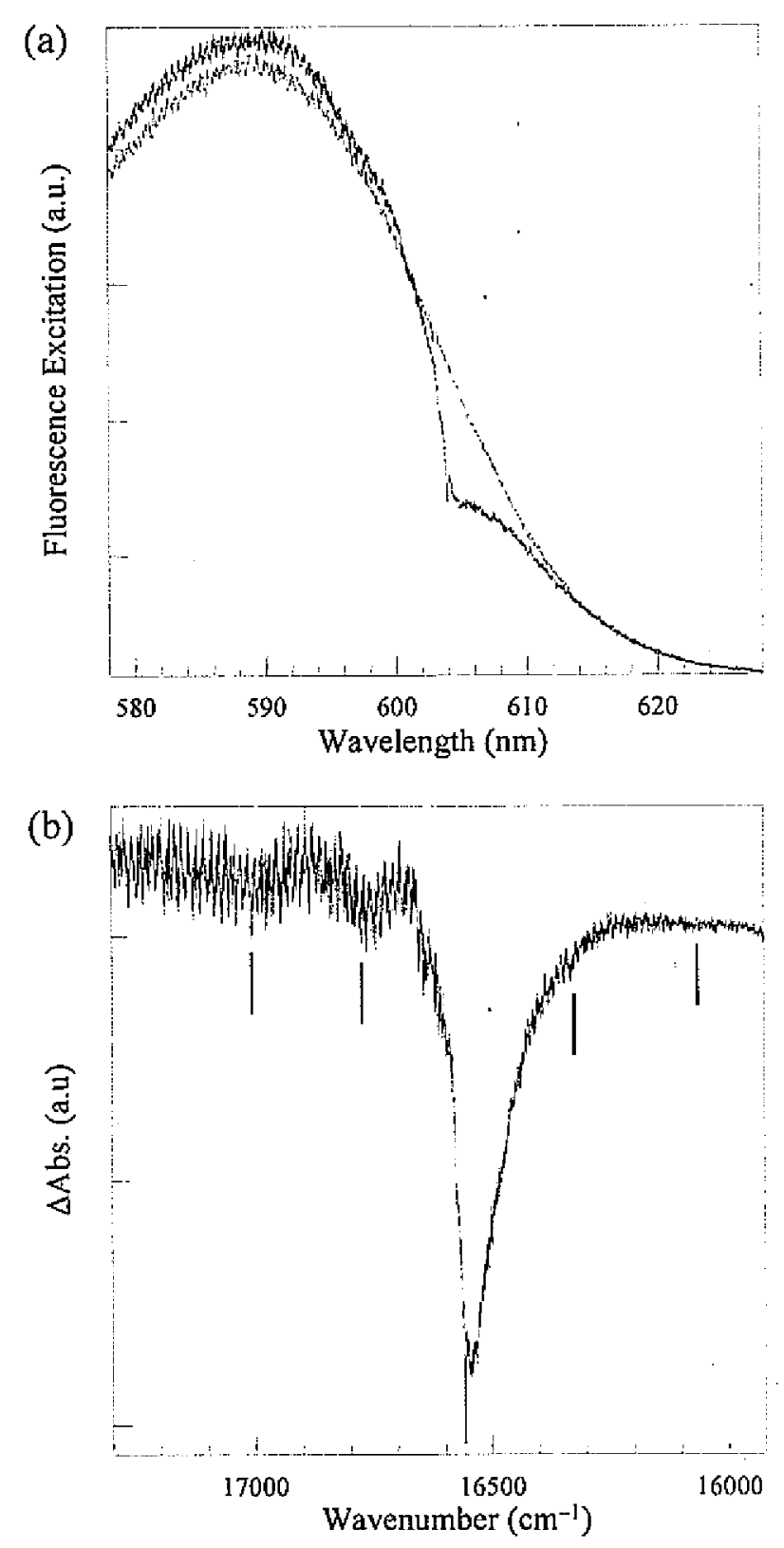

Figure A.3 (a) Pre- and post-burn spectra of CMXRos in the OV167 carcinoma cell line, burned at $604 \mathrm{~nm}$. Burn laser intensity was $50 \mathrm{~mW} / \mathrm{cm}^{2}$ for $20 \mathrm{~min}$ (for saturation). (b) The resultant hole spectra obtained from data presented in frame (a). Satellite vibronic holes can be seen on both sides of the hole at $\sim 220 \mathrm{~cm}^{-1}$ and at $\sim 460 \mathrm{~cm}^{-1}$, as indicated by solid lines. $\omega_{0}$ for this sample was determined to be $\sim 17 \mathrm{~cm}^{-1}$ (note, for VOSE14 normal cell line, $\omega_{0}$ was $\sim 24 \mathrm{~cm}^{-1}$ on same burn fluence). 
The hole growth kinetics curves of CMXRos in cells resembles those of MF680, where both sets showed faster burn rates in the OV167 carcinoma cell line than in the VOSE14 analogous normal cell line, thus implying the OV167 cellular matrix is more highly ordered [13]. The HGK curves in those cell lines at $603.5 \mathrm{~nm}$ (peak of the action spectra shown in Figure A.2) are shown in Figure A.4. Qualitative comparison of $\lambda_{0} s$ and $\sigma_{2} s$ obtained from fitting between these cell lines also resembled MF680 results. See figure caption for actual values calculated from fittings. On the other hand, so far Stark holeburning results of CMXRos in cells have not successfully reproduced the ratios in the permanent dipole moment change $(f \cdot \Delta \mu)$ of 1.5 [14] as was obtained with MF680. The cause of this discrepancy is still unknown.

Preliminary tissue experiments with CMXRos were performed. Fluorescence excitation spectra (at $5.2 \mathrm{~K}$ ) of tissues stained with $500 \mathrm{nM} \mathrm{CMXRos} \mathrm{for} 20 \mathrm{~min}$ at $37^{\circ} \mathrm{C}$, are presented in Figure A.5. As was noticed in the cell results, the absorption peak of the cancerous tissue showed a slight red-shift compared to that of the normal tissue. However, this difference is negligible. HGK curves at 3 different burning frequencies are also presented in Figure A.6. Even though these show the cancerous tissue burns slightly faster, it could simply be a defect in the sample, since concurrently performed experiments of identical tissue types with MF680 revealed almost no distinction between the HGK curves in cancerous and normal tissues (see Chapter 4 for further discussion).

In conclusion, CMXRos, as demonstrated by the spectroscopic results, has potential for application as another possible organelle specific probe in the hole burning imaging of cellular matrices. The next step will be to determine, with varying staining conditions of the probe, how much the above-mentioned binding mode to the mitochondrial SH group, affects 


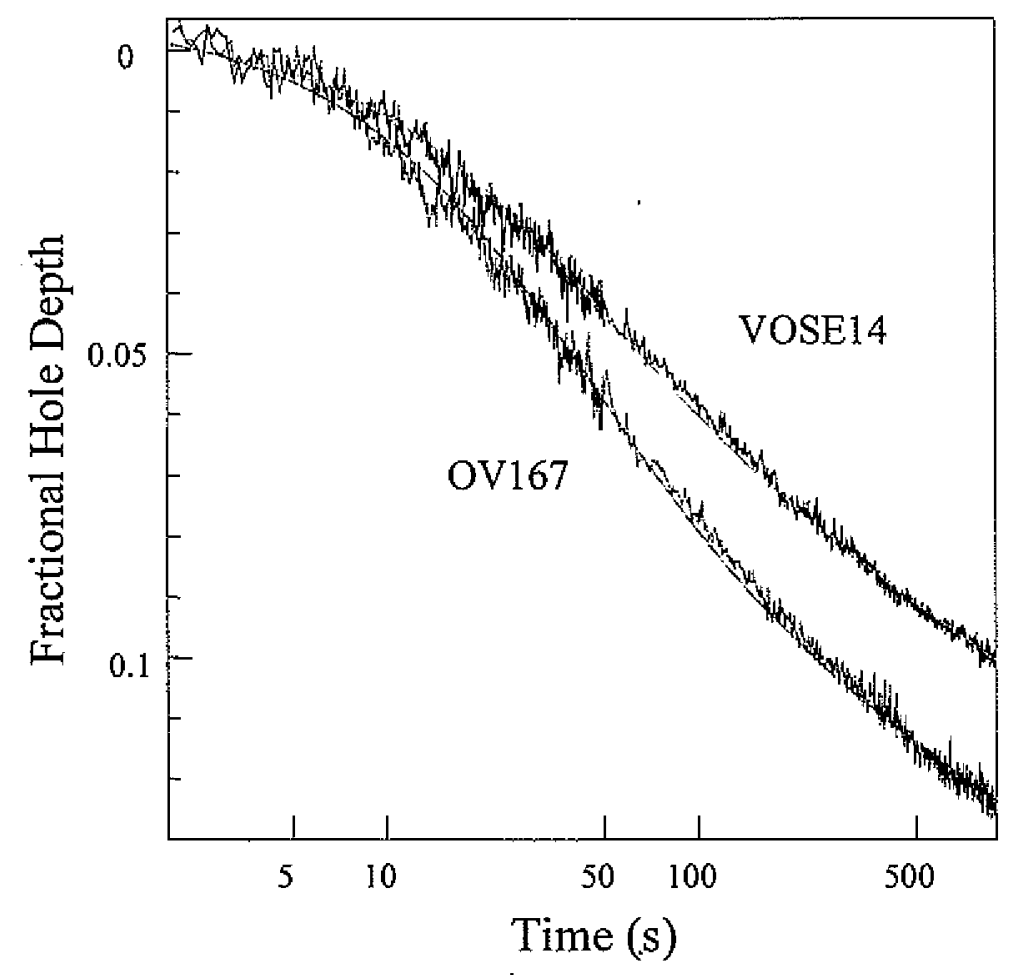

Figure A.4 Hole growth kinetics curves of CMXRos in ovarian surface epithelial cell lines OV167 (carcinoma) and VOSE14 (normal) at $603.5 \mathrm{~nm}$, shown with theoretical fittings. Burn fluence for both spectra was $5.2 \mu \mathrm{W} / \mathrm{cm}^{2}$. With a fixed value for Huang-Rhys factor, $S=1.7, \lambda_{0}$ and $\sigma_{2}$ were calculated to be 5.601 and 2.164 for OV167 and 6.383 and 2.388 for VOSE14, respectively. Note, for the sake of comparison, MF680 showed 7.78 and 0.85 for OV 167 and 8.36 and 1.10 for VOSE14, respectively [13] (at $710 \mathrm{~nm}$ ). 


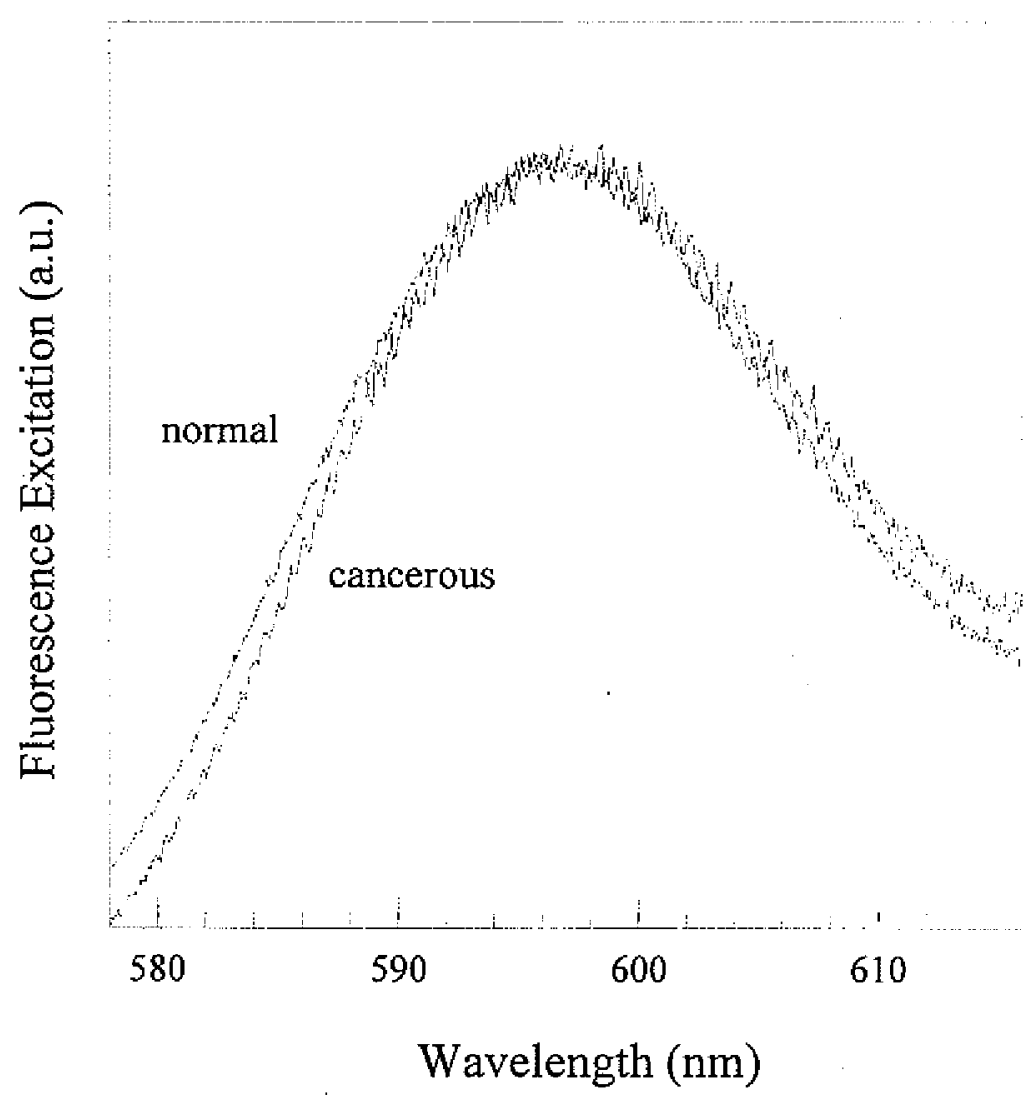

Figure A.5 Fluorescence excitation spectra of ovarian surface cancerous and normal peritoneal tissues stained with $500 \mathrm{nM} \mathrm{CMXRos,} \mathrm{measured} \mathrm{at} 5.2 \mathrm{~K}$. 

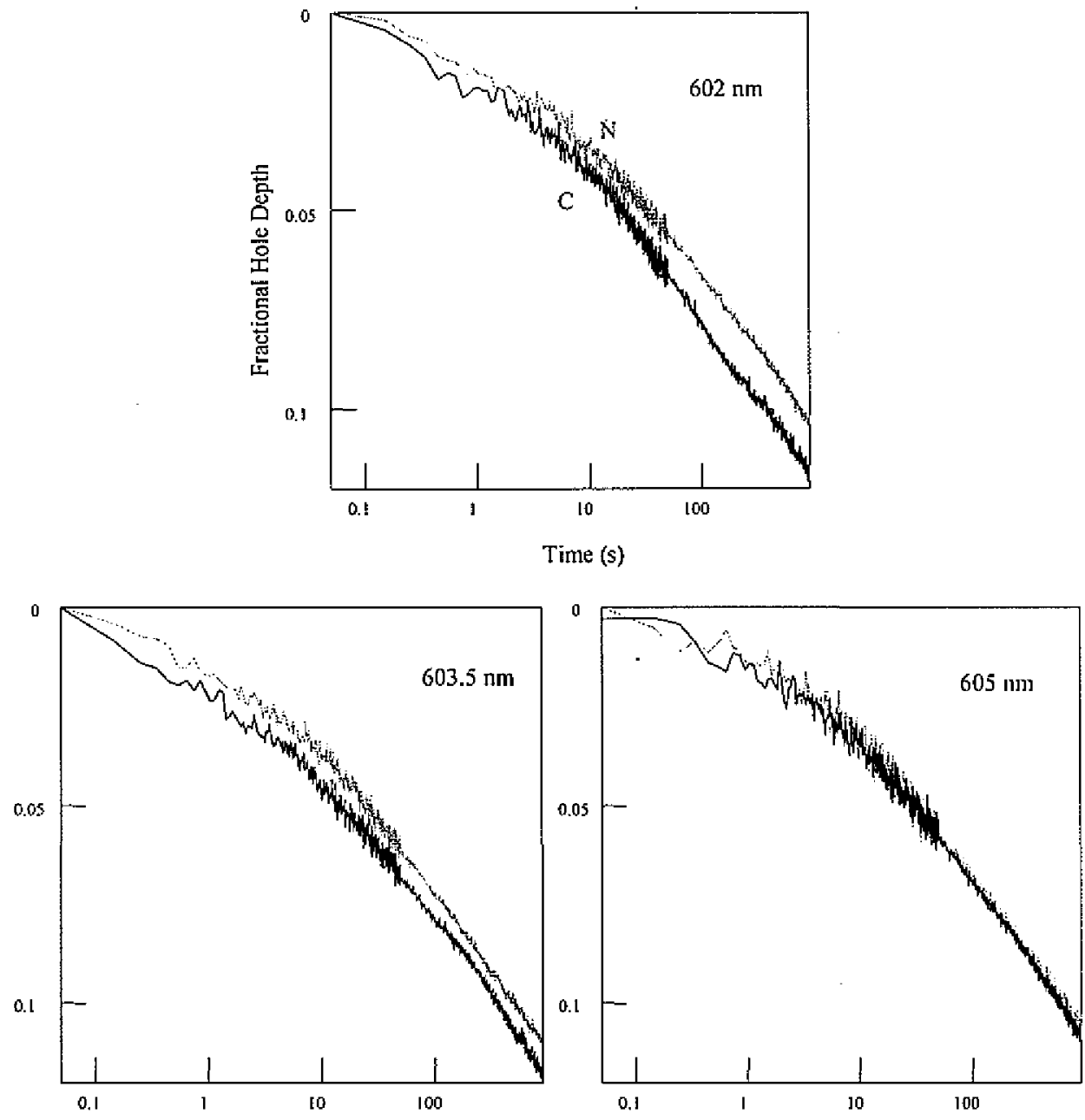

Figure A.6 Hole growth kinetics curves of CMXRos in ovarian surface cancerous tissue (C) and in analogous peritoneal normal tissue $(\mathrm{N})$ burned at three different wavelengths. These three wavelengths were chosen by their positions in the action spectra ( $603.5 \mathrm{~nm}$; measured with VOSE14 cell line, see Figure A.2) and $1.5 \mathrm{~nm}$ longer/shorter wavelengths. Burn fluence for all curves was 25.5 $\mu \mathrm{W} / \mathrm{cm}^{2}$. 
the Stark results. In addition, since the binding mode is suggested [11] irrespective of the magnitude of $\Delta \Psi_{\mathrm{m}}$, it must provide uniformity of surrounding environment, regardless of the $\Delta \Psi_{\mathfrak{m}}$, which is supposed to be more useful for further HGK studies of cellular matrices in cells and tissues.

\section{References:}

1. Buckman, J. F., Hernández, H., Kress, G. J., Votyakova, T. V., Pal, S., Reynolds, I. J. J. Neurosci. Meth., 2001, 104, 165.

2. Minamikawa, T., Sriratana, A., Williams, D. A., Bowser, D. N., Hill, J. S., Nagley, P. J. Cell Sci., 1999, 112, 2419.

3. Minamikawa, T., Williams, D. A., Bowser, D. N., Nagley, P. Exp. Cell Res., 1999, 246,26 .

4. Métivier, D., Dallaporta, B., Zamzami, N., Larochette, N., Susin, S. A., Marzo, I., Kroemer, G. Immunology Letters, 1998, 61, 157.

5. Macho, A., Decaudin, D., Castedo, M., Hirsch, T., Susin, S. A., Zamzami, N., Kroemer, G. Cytometry, 1996, 25, 333.

6. Krysko, D. V., Roels, F., Leybaert, L., D'Herde, K. J. Histchem. Cytochem., 2001, $49(10), 1277$.

7. Bassøe, C.-F., Li, N., Ragheb, K., Lawler, G., Sturgis, J., Robinson, J. P. Cytometry Part B, 2003, 51B, 21.

8. Mathur, A., Hong, Y., Kepm, B. K., Barrientos, A. A., Erusalimsky, J. D. Cardiovascular Research, 2000, 46, 126.

9. Rottenberg, H., Wu, S. BBA, 1998, 1404, 393.

10. Salvioli, S., Maseroli, R., Pazienza, T. L., Bobyleva, V., Cossarizza, A. Biochemistry (Moscow), 1998, 63(2), 277.

11. Scorrano, L., Petronilli, V., Colonna, R., Di Lisa, F., Bernardi, P. J. Biol. Chem., 1999, 274(35), 24657.

12. Reinot, T., Kim, W. H., Hayes, J. M., Small, G. J. J. Opt. Soc. Am. B, 1997, 14, 602. 
13. Walsh, R. J., Reinot, T., Hayes, J. M., Kalli, K. R., Hartmann, L. C., Small, G. J. Biophys. J., 2003, 84, 1299.

14. Walsh, R. J., Reinot, T., Hayes, J. M., Kalli, K. R., Hartmann, L. C., Small, G. J. J. Lumin., 2002, 98, 115. 


\section{APPENDIX B. FLOW CYTOMETRIC ESTIMATION OF MITOCHONDRIAL MEMBRANE POTENTIAL}

The most widely used flow cytometric application has been DNA content analysis. From the late 1960s, various DNA probes such as ethidium bromide [1] and propidium iodide (PI) [2] have been tested for cell-cycle analysis. It should be noted that both require treatment of the sample with RNAse to remove any residual double stranded RNA. PI is also frequently utilized in the dye exclusion test for cell viability. More recently, blue-fluorescent Hoechst 33342 [3,4], which binds to A-T triplets in DNA, replaced the above-mentioned DNA dyes. A typical cell-cycle analysis result is presented in Figure B.1. Note that cells in the "first gap", or $\mathrm{G}_{1}$ phase of the cell cycle, have a DNA content described as "diploid" or " $2 \mathrm{C}$ " in the figure, i.e. the amount of DNA contained in the 2 sets of chromosomes present before DNA replication begins. During DNA synthesis, or S phase, DNA content increases to twice this amount; the " $4 \mathrm{C}$ " values ("tetraploid"). It remains at $4 \mathrm{C}$ through the "second gap", or $\mathrm{G}_{2}$ phase, and during mitosis, the $\mathrm{M}$ phase. After mitosis, there are two daughter cells, each with a 2C DNA content. Cell-cycle analyses of OV167 carcinoma cell line and VOSE14 normal cell line revealed that both of the model cell lines are in a steady state, in terms of proliferation, at the time of harvesting. $75 \pm 5 \%$ of entire population were found in the $G_{0} / G_{l}$ quiescent state for OV167, where VOSE14 showed $60 \pm 5 \%$ (unpublished results).

Recently, not only for the cell-cycle analysis, but also these DNA probes have been rigorously used for the DNA content analysis with the labeled antibodies to examine certain antigens in conjunction with immunophenotyping.

As another frequently performed application of the flow cytometry, there is the membrane (cytoplasmic, mitochondrial or bacterial) potential $\left(\Delta \Psi^{\prime}\right)$ estimation. Indirect 
(a)

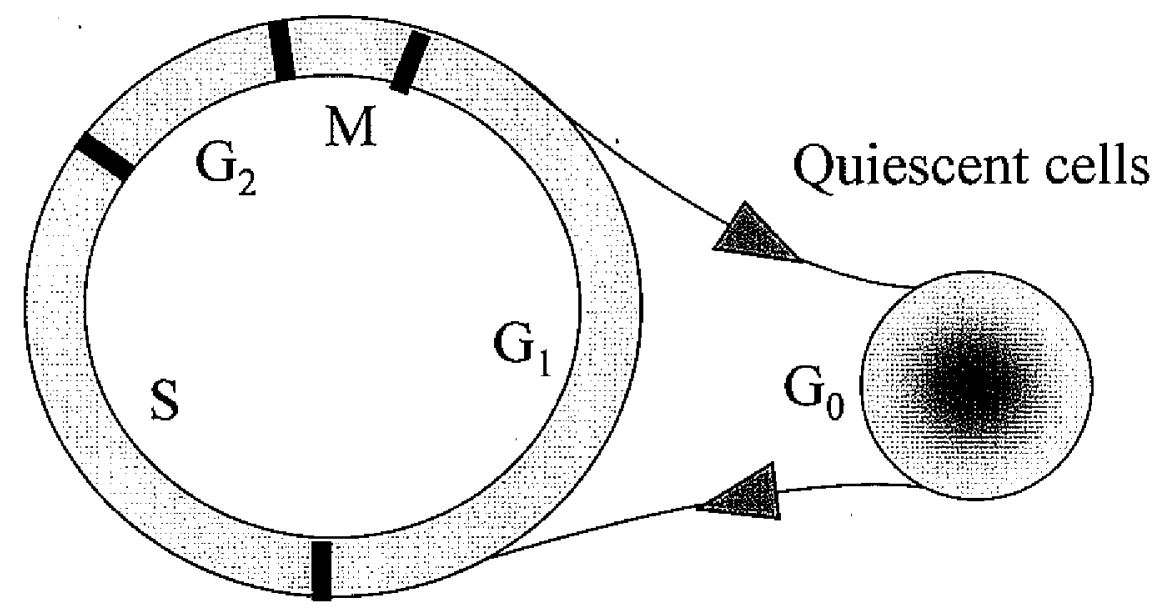

(b)

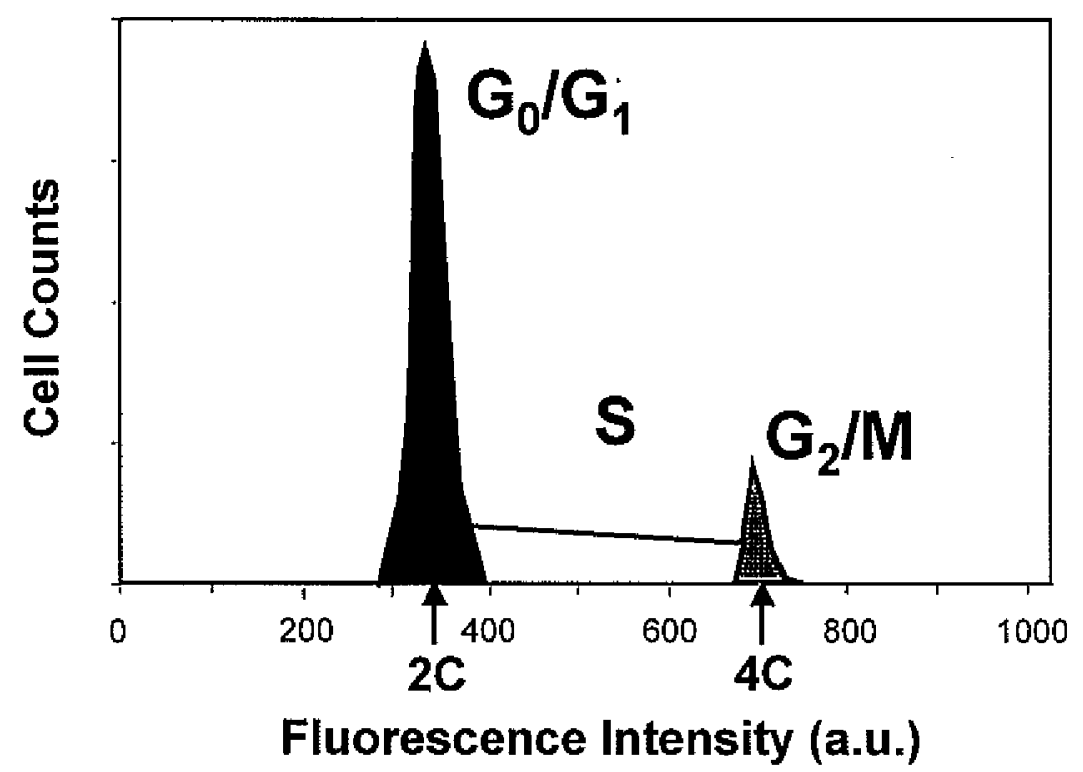

Figure B.1 (a) Cell-cycle diagram and (b) a typical flow cytometric result of cell-cycle analysis by DNA staining. Abscissa of part (b) represents the DNA contents of cells. See text for detailed discussion. In recent years, propidium iodide (PI) has been utilized as the fluorescence probe to analyze mainly fixed cells, while Hoechst 33342 has been applied to live cells (or for live cell sorting purposes). 
estimates of $\Delta \Psi$, in comparison to the direct membrane potential measurement (e.g. using implanted microelectrodes), can be obtained by monitoring the distribution of radiolabeled lipophilic cationic indicators such as 3H-triphenylmethylphosphonium [5] or lipophilic cationic dyes such as cyanines and safranines, between the cells and surrounding medium. Lipophilic indicators are used because innate characteristics enable these indicator molecules to pass freely through the lipid portion of the membranes. Thus, the concentration gradient of an indicator species, $\left[\mathrm{C}^{+}\right]$, across the membrane is determined by the potential difference across the membrane according to the Nernst equation:

$$
\left[\mathrm{C}^{+}\right]_{\mathrm{i}} /\left[\mathrm{C}^{+}\right]_{\mathrm{o}}=\exp (-\mathrm{FE} / \mathrm{RT}),
$$

where $\left[\mathrm{C}^{+}\right]_{\mathrm{i}}$ and $\left[\mathrm{C}^{+}\right]_{0}$ represent inner and outer concentrations of the indicator, respectively. A ratio $\left[\mathrm{C}^{+}\right]_{i} /\left[\mathrm{C}^{+}\right]_{0}$ of 10 corresponds to a potential difference of $61 \mathrm{mV}$ at $37^{\circ} \mathrm{C}[6]$. Once cells have been equilibrated with an indicator cation, depolarization of the cells will cause release of the indicator from the cells into the medium, while hyperpolarization will make cells take up additional indicator from the medium. While distributional probes may be suitable for detection of slow changes in $\Delta \Psi$, they cannot be used to monitor the faster changes that occur during the propagation of action potentials in tissue such as nerves and muscle [7].

Under normal circumstances, the intracellular concentration of $\mathrm{K}^{+},\left[\mathrm{K}^{+}\right]_{\mathrm{i}}$, is considerably higher than the extracellular concentration $\left[\mathrm{K}^{+}\right]_{0}$, while the intracellular concentration of $\mathrm{Na}^{+},\left[\mathrm{Na}^{+}\right]_{\text {, }}$, is considerably lower than the extracellular concentration $\left[\mathrm{Na}^{+}\right]_{0}$. The potassium-selective ionophore valinomycin, which is lipophilic, forms a complex with $\mathrm{K}^{+}$ions and can thus readily transport them across cell membranes. Addition of valinomycin thus effectively increases the cells' potassium permeability, to the point at 
which the membrane potential is determined almost entirely by the transmembrane $\left[\mathrm{K}^{+}\right]$ gradient. If $\left[\mathrm{K}^{+}\right]_{0}$ is low, valinomycin addition hyperpolarizes cells; if $\left[\mathrm{K}^{+}\right]_{0}$ is high, valinomycin addition depolarizes cells [8].

Estimation of $\Delta \Psi$ of cells in a suspension using symmetric cyanine dye [9] was first attempted by fluorometers. Addition of cells to micromolar solutions of dye such as dihexyloxacarbocyanine $\left(\mathrm{DiOC}_{6}(3)\right)$ produces a suspension with lower fluorescence than that of the original solution, indicating that at micromolar external concentrations, the fluorescence of dye taken into cells is quenched. More recently, Rottenburg and Wu reported [10] that $\mathrm{DiOC}_{6}(3)$ could be used for quantitative flow cytometric measurements of $\Delta \Psi_{\mathrm{m}}$, though the authors noted in 1997 [11] that $\mathrm{DiOC}_{6}(3)$ itself induced apoptotic changes in lymphocytes, which led them to consider some attributes of this and other dyes might affect their use as probes of $\Delta \Psi_{\mathrm{m}}$.

The current trend of flow cytometric estimation of $\Delta \Psi_{\mathrm{m}}$ is the rationetric technique, which is well described in Ref. [12]. This trend started in 1991, when Lan Bo Chen and coworkers described the use of the symmetric cyanine dye $5,5^{\prime}, 6,6^{\prime}$-tetrachloro-1, ', $, 3,3^{\prime}$ tetraethylbenzimidazolylcarbocyanine iodide (JC-1), which forms fluorescent aggregates as a probe of $\Delta \Psi_{\mathrm{m}}[13,14]$. When excited at $490 \mathrm{~nm}$ (or more commonly $488 \mathrm{~nm}$ by argon ion laser), $\mathrm{JC}-1$ monomers show an emission maximum at $527 \mathrm{~nm}$, while aggregates emit maximally at $590 \mathrm{~nm}$. In theory, the orange to green fluorescence ratio should yield a result for $\Delta \Psi_{\mathrm{m}}$. Typical experimental schematic of flow cytometer is described in Figure B.2. More recently, JC-9, a dibenzo homologue of $\mathrm{DiOC}_{2}(3)$ that shares the tendency of JC-1 to form fluorescent $\mathrm{J}$-aggregates and supposedly has better separation of aggregate signals from monomer signals than JC-1 [15], was developed again by Molecular Probes (Eugene, OR). 


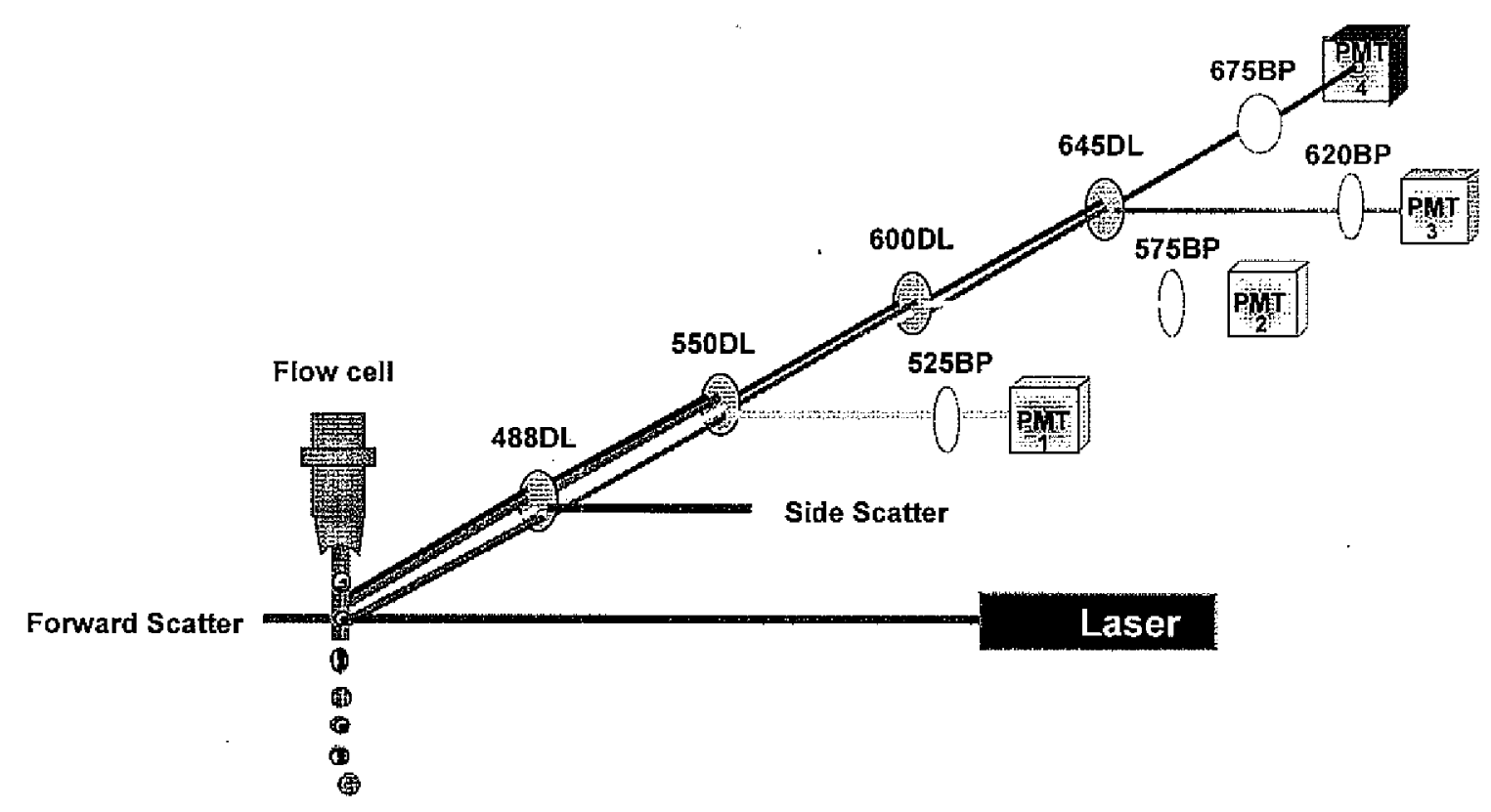

Figure B.2 Diagram of typical Coulter EPICS ${ }^{\circledast}$ XL-MCL optics configuration. For the mitochondrial membrane potential estimation with JC-9, the fluorescence ratio was obtained from signals detected by PMT1 (green) and PMT3 (orange/red). See text for details. Note that forward and side scatter signals, which represent size and granularity [7], respectively, were used to gate dead and apoptotic cells out of the entire cell populations. In the figure, DL stands for the dichroic filters while BS is for the bandpass filters. 
JC-9 red/green fluorescence ratio profiles with various $\left[\mathrm{K}^{*}\right]_{0}$ in the OV167 cell line are presented in Figure B.3.

Measurements to determine $\Delta \Psi_{m}$ for each cell line were performed using the JC-9 probe by following the procedures for semi-quantitative measurement of $\Delta \Psi_{\mathrm{m}}$ documented by Rottenburg and Wu [10]. Briefly, for each of OV167 carcinoma and VOSE14 normal cell lines, the fluorescence ratios (red/green) were calibrated by various phosphate buffered saline with different $\mathrm{KCl}$ concentrations (ranging from 0.1 to $180 \mathrm{mM}$ ), in the presence of valinomycin $(2 \mu \mathrm{M})$, to the membrane potential scale. The baseline fluorescence ratio was obtained from the $\Delta \Psi_{\mathfrak{m}}$-depolarized sample, which is achieved by the incubation of the cells with $100 \mu \mathrm{M}$ of the protonophore carbonyl cyanide $m$-chlorophenyl-hydrazone (CCCP) for a half hour after the dye (JC-9) loading. The $\Delta \Psi_{\mathrm{m}}$ values were determined by using the calibration curve for the baseline-corrected fluorescence ratio of the positive control (stained only by JC-9). The values for OV167 carcinoma cell line and VOSE14 normal cell line were measured to be (negative inside) $-199.7 \pm 16.4 \mathrm{mV}$ and $-133.7 \pm 34.8 \mathrm{mV}$, respectively. The $\Delta \Psi_{\mathrm{m}}$ ratio measured by flow cytometry between the two cell lines is 1.493 , which is in good agreement with the $f \cdot \Delta \mu$ ratio given by Stark hole-burning ( 1.5) [16]. See Refs. [17-21] for further discussion of the $\sim 1.5$-fold higher $\Delta \Psi_{\mathrm{m}}$ 's observed in cancerous cell lines. Note that the standard error corresponding to $\Delta \Psi_{\mathrm{m}}$ of the normal cells is twice as large, due to the inconsistent growth rate of the cell line.

\section{References:}

1. Dittrich, W., Göhde, W. Z Naturforsch, 1969, 24b, 360.

2. Crissman, H. A., Steinkamp, J. A. J. Cell Biol., 1973, 59, 766. 


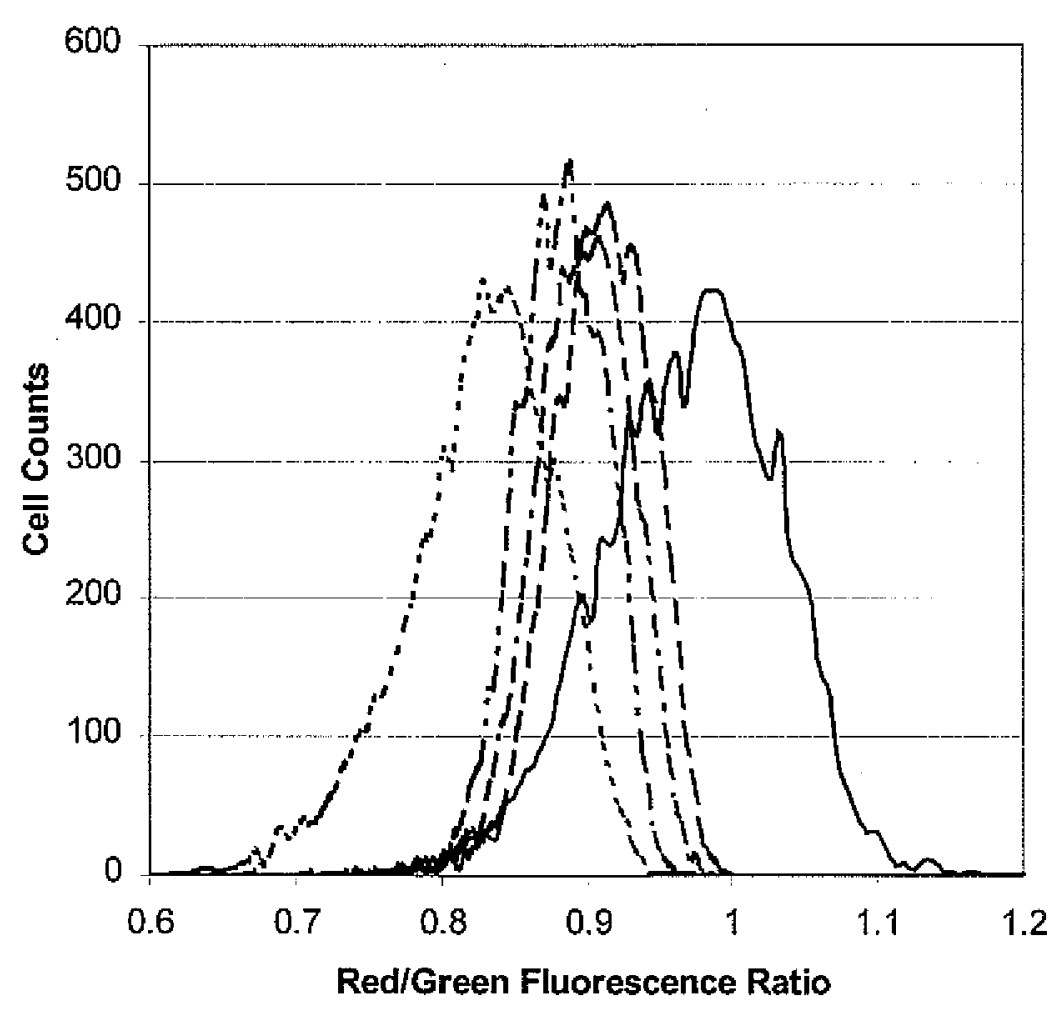

Figure B.3 JC-9 fluorescence ratio profiles obtained for OV167 carcinoma cell line (measured at Cell and Hybridoma Facility, a branch of Office of Biotechnology, Iowa State University). The solid line represents a positive control, which was stained only by JC- $9\left(2 \times 10^{-5} \mathrm{M} ; 10 \mathrm{~min}\right.$ incubation at 37 ${ }^{\circ} \mathrm{C}$ ), while the dotted line expresses a JC-9 ratio profile of depolarized (baseline) $\Delta \Psi_{\mathrm{m}}$ by an uncoupler CCCP $\left(1 \times 10^{-5} \mathrm{M} ; 30 \mathrm{~min}\right.$ incubation at 37 ${ }^{\circ} \mathrm{C}$ ). Dashed lines were achieved by the adjustment of $\left[\mathrm{K}^{+}\right]_{0}$ in the presence of a $\mathrm{K}^{+}$-ionophore valinomycin $\left(2 \times 10^{-6} \mathrm{M} ; 15 \mathrm{~min}\right.$ incubation at $\left.37^{\circ} \mathrm{C}\right)$ to give certain membrane potential values derived according to the Nernst equation. The dashed line, dashed line with one dot and dashed line with two dots were measured in the buffers containing $8.6 \mathrm{mM}, 15.9 \mathrm{mM}$, and $45.2 \mathrm{mM} \mathrm{K}^{+}$, respectively. The fluorescence ratio values at each condition (to give $\Delta \Psi_{\mathrm{m}}$ values described in text) were obtained from the statistical average of individual distributions. 
3. Latt, S. A., Stetten, G. J. Histochem. Cytochem., 1976, 24, 24.

4. Arndt-Jovin, D. J., Jovin, T. M. J. Histochem. Cytochem., 1977, 25, 285.

5. Bakeeva, L. E., Grinius, L. L., Jasaitis, A. A. $B B A$, 1970, 216, 13.

6. Sims, P. J., Waggoner, A. S., Wang, C.-H. Biochemistry, 1974, 13, 3315.

7. Shapiro, H. M. Practical Flow Cytometry $\left(4^{\text {th }}\right.$ ed.), 2003, Wiley-LISS

8. Waggoner, A. S. Ann. Rev. Biophys. Bioeng., 1979, 8, 47.

9. Hoffman, J. F., Laris, P. C. J. Physiol., 1974, 239, 519.

10. Rottenberg, H., Wu, S. $B B A, \mathbf{1 9 9 8 , 1 4 0 4 , 3 9 3 .}$

11. Rottenberg, H., Wu, S. Biochem. Biophys. Res. Commun., 1997, $240,68$.

12. Novo, D., Perlmutter, N. G., Hunt, R. H., Shapiro, H. M. Cytometry, 1999, 35, 55.

13. Reers, M., Smith, T. W., Chen, L. B. Biochemistry, 1991, 30, 4480.

14. Cossarizza, A., Baccarani-Contri, M., Kalashnikova, G., Franceschi, C. Biochem. Biophys. Res. Commun., 1993, 197, 40.

15. Haugland, R. P. Handbook of Fluorescent Probes and Research Products $\left(9^{\text {th }}\right.$ ed.), 2002, Molecular Probes Inc.

16. Walsh, R. J., Reinot, T., Hayes, J. M., Kalli, K. R., Hartmann, L. C., Small, G. J. .J. Lumin., 2002, 98, 115.

17. Johnson, L.V., Walsh, M. L., Chen, L. B. Proc. Natl. Acad. Sci. USA, 1980, 77, 990.

18. Summerhayes, I. C., Lampidis, T. J., Bernal, S. D., Nadakavukaren, J. J., Nadakavukaren, K. K., Shepherd, E. L., Chen, L. B. Proc. Natl. Acad. Sci USA, 1982. $79,5292$.

19. Modica-Napolitano, J. S., Aprille, J. R. Cancer Res., 1987, 47, 4361.

20. Davis, S., Weiss, M. J., Wong, J. R., Lampidis, T. J., Chen, L. B. J. Bio. Chem., 1985, $260,13844$.

21. Dairkee, S. H., Hackett, A. J. Breast Cancer Res. Treat., 1991, $18,57$. 


\section{APPENDIX C. CRYOPRESERVATION}

To ensure reproducible results and continuity in research and biomedical processes, today's researchers are faced with the task of genetically stabilizing living cells. Serial subculturing of any cell type is time consuming and can lead to contamination or genetic drift as smaller and smaller portions of a population are selected. However, cell population can be stabilized by subjugation to cryogenic temperatures, which, for all practical purposes, stops time. The stabilization of cells at a cryogenic temperature is called cryopreservation, an applied aspect of cryobiology. Advances in cryopreservation technology have led to methods that allow low-temperature maintenance of a variety of cell types. Low temperatures provide ultrastructural information about tissues and cells unobtainable at room temperature. During cryopreservation, the dynamics of structures in a cellular system are frozen-out, facilitating an unobstructed view of cellular content. Moreover, since nonphotochemical hole-burning requires cryogenic temperatures to obtain high-resolution spectra, low temperature effects on cellular systems must be considered and, subsequently, appropriate sample preparation procedures devised for spectroscopic studies under such conditions. Therefore, this section will outline the cryopreparative procedures routinely used for low-temperature storage and ultrastructural analysis.

The fundamental problem confronting cryobiology comes from the high intrinsic water content in cells $(\sim 70 \%)$. Upon freezing, the normal phase transition of water is from a liquid at ambient temperature to ice at subambient temperatures. The problem of ice crystal formation at cryogenic temperatures stems from the fact that ice forms at different rates during the cooling process. During cooling, freezing occurs external to the cell before intracellular ice begins to form [1]. As ice forms, water is removed from the extracellular 
environment and an osmotic imbalance occurs across the cell membrane leading to water migration out of the cell. The increase in solute concentration outside the cell, as well as intracellularly, can be detrimental to cell survival [1]. If too much water remains inside the cell, damage due to ice crystal formation as well as recrystallization can occur during the warming (thawing) process.

The rate of cooling has a dramatic effect on this ice formation phenomenon. Rapid cooling minimizes the osmotic imbalance as ice forms uniformly, but this leads to more intracellular ice. Slow cooling, on the other hand, results in a greater loss of water from the cell and less internal ice, but increases the osmotic imbalance. Cell permeability affects the rate of water loss; more permeable cells are able to tolerate rapid cooling better than less permeable cells [2]. Mazur et al. [3] have postulated that ice crystal formation and solution effects both play a role in cell inactivation, and that finding an optimum cooling rate can minimize the effect of each. Although different types of cells may require different cooling rates, a uniform cooling rate of $1 \mathrm{~K}$ per minute from ambient temperature has been found to be effective for a wide variety of cells. To achieve a uniform, controlled cooling rate, the preferred method is to use a programmable-rate cell freezing apparatus. However, inexpensive refrigeratable containers that can satisfy these conditions are commercially available. Nalgene's $1^{\circ} \mathrm{C}$ freezing container, for example, can reliably provide a rate of cooling very close to $1^{\circ} \mathrm{C}$ per minute.

Using cryoprotective additives or chemicals that protect the cells during freezing can also minimize the detrimental effects of increased solute concentration and ice crystal formation. The most commonly used cryoprotective agents are dimethylsulfoxide (DMSO) and glycerol. For most cells, glycerol is the agent of choice becuase it is usually less toxic 
than DMSO. However, DMSO is more penetrating and is usually the agent of choice for larger, more complex cells such as protists. It should be noted that the toxicity of a cryoprotective agent is dependent on its concentration, the ambient temperature, and time of exposure.

A low-temperature microscopic study of various freezing procedures performed on Chinese hamster fibroblasts by Farrant et. al. [4] showed that rapid cooling of small cell samples with $5 \%$ DMSO $(\mathrm{v} / \mathrm{v})$ resulted in acceptable levels of ice formation, satisfactory ultrastructure, and no shrinkage. The disadvantage to this procedure is that, upon thawing, the cells are no longer viable. Therefore, even though this approach is adequate for ultrastructural studies, it is not suitable as a protocol for cryogenic storage. For storage purposes, the authors proposed a simple two-step procedure. This involves cooling the sample at the aforementioned cooling rate of $\sim 1 \mathrm{~K} / \mathrm{min}$ down to ca. $-70^{\circ} \mathrm{C}$. The cells are then rapidly cooled to liquid nitrogen temperature. An electron micrograph of the Chinese hamster fibroblasts cooled in this manner were shrunken, but showed no ice formation [4]. The reader interested in further discussions on various freezing procedures should refer to the recent reviews by Wisniewski [5] and/or by Wolfinbarger [6].

As of yet, no precedent has been established regarding these preparations in relation to hole burning studies of cellular systems. The rapid cooling regime would certainly provide better preservation of cellular structure. However, since ice is not present under the two-step cooling scheme, conditions are imposed for a nonphotochemical hole-burning system with a probe in a disordered matrix [7]. As matter of fact, many of the probe molecules for cellular matrices, such as APT, which result in hole widths of hundreds of megahertz in amorphous water, will not hole burn in ice [8]. Nonetheless, the shrunken state 
of a cell having undergone two-step cooling may hinder efforts to differentiate between cell types based on compressibility. In Chapter 5, tissue samples were prepared in the presence of a cryopreservative followed by two-step cooling.

\section{References:}

1. Farrant, J. in Low Temperature Preservation in Medicine and Biology (AshwoodSmith, M. J. and Farrant, J. eds.), 1980, Pitman Medical Limited, Kent, England.

2. Nei, T., Araki, T., Matsusaka, T. in Freezing and Drying of Microorganisms (Nei, T. ed.), 1969, University of Tokyo Press, Tokyo, Japan.

3. Mazur, P., Leibo, S. P., Chu, E. H. Y. Exp. Cell Res., 1972, 71, 345.

4. Farrant, J, Walter, C. A., Lee, H., Morris, G. J., Clarke, K. J. J. Microscopy, 1977, $111,17$.

5. Wisniewski, R. BioPharm, 1998, 11, 35.

6. Wolfinbarger, Jr. L. BioPharm, 1998, 11, 42.

7. Milanovich, N. Ph.D. Dissertation, Iowa State University, 1999.

8. Kim, W.-H., Reinot, T., Hayes, J. M., Small, G. J. J. Phys. Chem., 1995, 99, 7300. 


\section{ACKNOWLEDGMENTS}

I would like to express my thanks to a number of persons who have aided me in the performance of various stages of this research project. I am indebted to my research advisors, Professors Gerald Small and Ryszard Jankowiak, for their guidance, patience and numerous discussions in the past few years. I am fortunate to benefit from their knowledge and insights in various subjects.

Dr. John Hayes deserves my sincere appreciation and much credit for the work presented in this thesis. I am also particularly grateful to Dr. Tõnu Reinot for his various suggestions to this thesis work and providing me some great figures. I am also grateful to Dr. Valter Zazubovic for his assistance and expertise.

I am particularly thankful to Dr. Richard Walsh and (soon to be Drs.) Mr. Nhan Dang and Mr. Kerry Riley for helpful discussions. Most of all, my sincere thank goes to Mr. Matt Pinkston who spent hours proofreading my writing.

The United States Government has assigned the DOE Report number IS-T 2692 to this thesis. Notice: This document has been authored by the Iowa State University of Science and Technology under Contract No. W-7405-ENG-82 with the U.S. Department of Energy. The U. S. Government retains a non-exclusive, paid-up, irrevocable, world-wide license to publish or reproduce the published form of this document, or allow others to do so, for U. S. Government purposes. 